\title{
NHC-Mediated Stetter-Aldol and Imino-Stetter-Aldol Domino Cyclization to Naphthalen-1 (2H)-ones and Isoquinolines
}

\author{
Debabrata Barman, ${ }^{\text {aq }}$ Tanmoy Ghosh, ${ }^{\text {aq }}$ Krishanu Show, ${ }^{a}$ Sudipto Debnath, ${ }^{\text {a }}$ Tapas Ghosh, ${ }^{\mathrm{b}}$ and \\ Dilip K. Maiti*a \\ a Department of Chemistry, University of Calcutta, University College of Science, \\ 92, A. P. C. Road, Kolkata-700009, India \\ ${ }^{\mathrm{b}}$ Department of Applied Sciences, Maulana Abul Kalam Azad University of Technology, Haringhata- \\ 741249, West Bengal, India \\ *Corresponding author. Fax: 91-33-2351 9755, Tel: 91-33-2350 1014 \\ dkmchem@caluniv.ac.in "Equal contribution
}

Serial No.

Content

$\underline{\text { Page Numbers }}$

1. Section I: General information

S-2

2. Section II: General procedure for the synthesis of disubstituted naphthalen-1(2H)-one derivatives (GP-1) and characterization data of compounds $\mathbf{6 a - t}$

3. Section III: Optimization Table, general procedure for the synthesis of 3-aryl substituted isoquinolines (GP-2) and characterization data of compounds $\mathbf{9 a - p}$

4. Section IV: ESI-MS data for detecting the intermediates during the formation of $\mathbf{6 i}$

5. Section V: ESI-MS data for detecting the intermediates during the formation of $\mathbf{9 b}$

6. Section VI: ${ }^{1} \mathrm{H}$ and ${ }^{13} \mathrm{C}$-NMR spectra of substituted naphthalen-1(2H)-one derivatives (6a-t)

7. Section VII: ${ }^{1} \mathrm{H}$ and ${ }^{13} \mathrm{C}-\mathrm{NMR}$ spectra of substituted isoquinolines derivatives (9a-q)

8. Section VIII: X-ray characterization data summery of S-60 compound 6e (CCDC 2062484).

9. Section IX: X-ray characterization data summery of compound 9b (CCDC 1923004). 


\section{Section I: General Information}

All reagents were purchased from commercial suppliers and used without further purification, unless otherwise specified. Commercially supplied ethyl acetate and petroleum ether were distilled before use. All solvents were dried through usual methods. The petroleum ether used in our experiments having a boiling range $60-80{ }^{\circ} \mathrm{C}$. Analytical thin-layer chromatography was performed on $0.25 \mathrm{~mm}$ extra-hard silica gel plates with UV254 fluorescent indicator. The reported melting points are uncorrected. ${ }^{1} \mathrm{H}$ NMR and ${ }^{13} \mathrm{C}$ NMR spectra were recorded at ambient temperature using both $300 \mathrm{MHz}$ and $400 \mathrm{MHz}$ spectrometers $\left(300 \mathrm{MHz}\right.$ for ${ }^{1} \mathrm{H}$ and $75 \mathrm{MHz}$ for ${ }^{13} \mathrm{C}$, similarly $400 \mathrm{MHz}$ for ${ }^{1} \mathrm{H}$ and $100 \mathrm{MHz}$ for ${ }^{13} \mathrm{C}$ ). Chemical shifts are reported in ppm with respect to tetramethylsilane internal reference, and coupling constants are reported in Hz. Proton multiplicities are represented as s (singlet), d (doublet), dd (double doublet), $\mathrm{t}$ (triplet), $\mathrm{q}$ (quartet), and $\mathrm{m}$ (multiplet). Infrared spectra were recorded on an FT-IR spectrometer in thin films. HR-MS data were recorded on a Q-tof-micro quadruple mass spectrophotometer.

Section II: General procedure for the synthesis of disubstituted-naphthalen-1( $2 \mathrm{H})$-ones derivatives (GP-1): Under nitrogen atomsphere, phthalaldehyde (5a, $1.0 \mathrm{mmol}$ ) and $N$-tosyl- $\alpha, \beta$-unsaturated ketimines $^{1}(1,1.0 \mathrm{mmol})$ were added in dry DCM $(5 \mathrm{~mL})$ in the presence of thiazolium bromide 11c $(15$

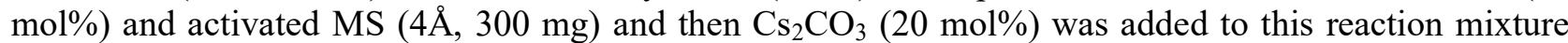
and stirred at ambient temperature for 4-8 h. Upon completion (monitored through TLC), the reaction mixture was filtered through the celite bed and the filtrate was extracted with DCM $(2 \times 15 \mathrm{~mL})$. The combined organic layer was washed with water $(3 \times 10 \mathrm{~mL})$ and dried over anhydrous $\mathrm{Na}_{2} \mathrm{SO}_{4}$, filtered and evaporated in a rotary evaporator under reduced pressure. The residue was chromatographed on a silica gel column (100-200 mesh) using ethyl acetate-petroleum ether $(10 \%$ to $20 \%, \mathrm{v} / \mathrm{v})$ as an eluent, which afforded the corresponding disubstituted- naphthalen-1(2H)-ones derivatives.

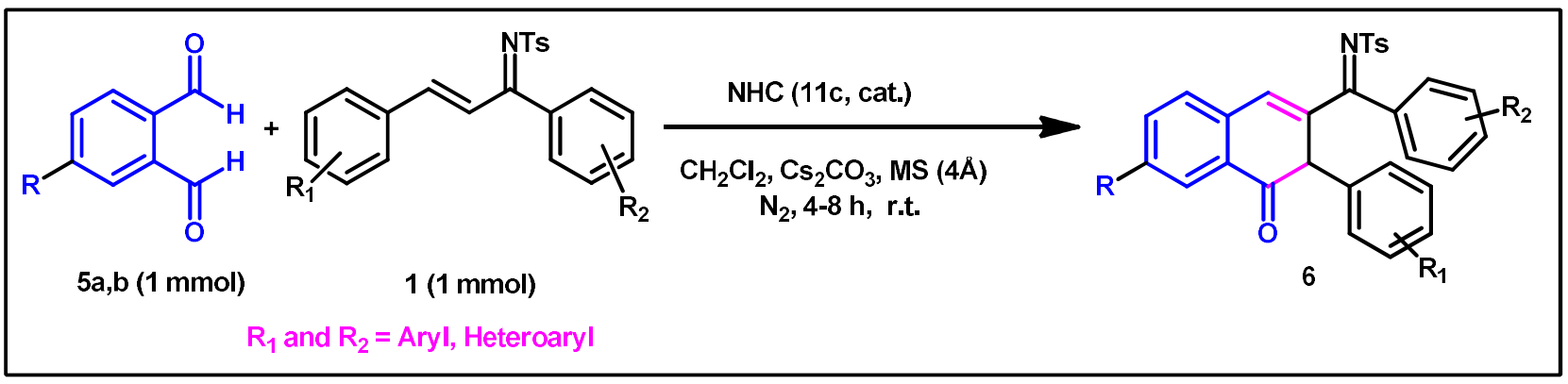

\section{Section IIa. Characterization Data of Compounds 6a-t:}

4-Methyl- $N$-((4-oxo-3-phenyl-3,4-dihydronaphthalen-2yl)(phenyl)methylene)benzenesulfonamide (6a): Compound 6a, was prepared following the GP-1 using phthalaldehyde (5a, $1 \mathrm{mmol})$ and $N$-((E)-1,3-diphenylallylidene)-4-methylbenzenesulfonamide (1a, $1 \mathrm{mmol}$ ) as starting material and afforded the title compound 6a (using 10\% ethyl acetatepetroleum ether (v/v) as an eluent for purification) as yellow thick liquid, Yield: $74 \%(352.0 \mathrm{mg}) ;{ }^{1} \mathbf{H}$ NMR (400 MHz, $\left.\mathbf{C D C l}_{3}\right): \delta$ 8.36-8.34 (m, 1H), 7.95-7.93 (m, 3H), $7.82(\mathrm{~s}, 1 \mathrm{H}), 7.64-7.62(\mathrm{~m}, 2 \mathrm{H})$, 7.38-7.25 (m, 9H), 7.19-7.10 (m, 3H), $5.75(\mathrm{~s}, 1 \mathrm{H}), 2.47(\mathrm{~s}, 3 \mathrm{H}) ;{ }^{13} \mathbf{C}$ NMR (100 MHz, CDCl $\left.\mathbf{3}\right): \delta$ $179.3,148.4,143.5,138.7,137.7,134.6,133.8,132.8,130.8,129.7,129.5,129.1,128.6,128.3$, 127.9, 127.4, 127.36, 127.0, 125.0, 122.7, 121.0, 119.0, 21.7; FT-IR (neat, $\mathrm{cm}^{-1}$ ): 3497, 3097, 2698, 1897, 1732, 1612, 1132, 680; HRMS (ESI-TOF) $\mathrm{m} / \mathrm{z}$ Calcd for $\mathrm{C}_{30} \mathrm{H}_{24} \mathrm{NO}_{3} \mathrm{~S}[\mathrm{M}+\mathrm{H}]^{+}: 478.1477$, found 478.1473 .<smiles>[NH2+]=C(C1=Cc2ccccc2C(=O)C1c1ccccc1)c1ccccc1</smiles> 
4-Methyl- $N$-((4-oxo-3-(p-tolyl)-3,4-dihydronaphthalen-2-

yl)(phenyl)methylene)benzenesulfonamide (6b): Compound $\mathbf{6 b}$ was prepared following the GP-1 using phthalaldehyde (5a, $1 \mathrm{mmol})$ and 4-methyl- $N-((E)-1-$ phenyl-3- $(p-$ tolyl)allylidene)benzenesulfonamide $(\mathbf{1 b}, 1 \mathrm{mmol})$ as starting material and afforded the title compound $\mathbf{6 b}$ (using 15\% ethyl acetate-petroleum ether (v/v) as an eluent for purification) as pale yellow solid, Yield: 78\% (382.0 mg); mp 139-140 ${ }^{\circ} \mathrm{C}$; ${ }^{1} \mathbf{H}$ NMR (400 $\left.\mathbf{M H z}, \mathbf{C D C l}_{\mathbf{3}}\right): \delta$ 8.34-8.32 (m, 1H), 7.93-7.91 (m, 3H), 7.77 (s, 1H), 7.63-7.61 (m, 2H), 7.37-7.29 (m, 3H), 7.28-7.22 (m, 3H), 7.15$7.11(\mathrm{~m}, 3 \mathrm{H}), 7.00(\mathrm{~m}, 2 \mathrm{H}), 5.70(\mathrm{~s}, 1 \mathrm{H}), 2.47(\mathrm{~s}, 3 \mathrm{H}), 2.26(\mathrm{~s}, 3 \mathrm{H}) ;{ }^{13} \mathbf{C}$ NMR (100 MHz, CDCl $\left.\mathbf{3}\right): \delta$ 179.2 , 148.4, 143.4, 138.6, 138.0, 137.6, 134.6, 132.6, 130.5, 129.6, 129.4, 128.5, 127.8, 127.3, 127.2, 126.8, 124.8, 122.6, 120.7, 118.9, 21.6, 21.1; FT-IR $\left(\mathrm{KBr}, \mathrm{cm}^{-1}\right): 3491,3372,3143,2903$, 2046, 1914, 1712, 1654, 1068, 543; HRMS (ESI-TOF) $\mathrm{m} / \mathrm{z}$ Calcd for $\mathrm{C}_{31} \mathrm{H}_{26} \mathrm{NO}_{3} \mathrm{~S}[\mathrm{M}+\mathrm{H}]^{+}$: 492.1633, found 492.1628 .<smiles></smiles>

(R)- $N$-((3-(4-Methoxyphenyl)-4-oxo-3,4-dihydronaphthalen-2-yl)(phenyl)methylene)-4-

methylbenzenesulfonamide (6c): Compound $\mathbf{6 c}$ was prepared following the GP-1 using phthalaldehyde (5a, $1 \mathrm{mmol})$ and $N$-((E)-3-(4-methoxyphenyl)-1-phenylallylidene)-4methylbenzenesulfonamide (1c, $1 \mathrm{mmol})$ as starting material and afforded the title compound $\mathbf{6 c}$ (using $14 \%$ ethyl acetate-petroleum ether $(\mathrm{v} / \mathrm{v})$ as an eluent for purification) as brownish viscous liquid, Yield: 73\% (370.0 mg); ${ }^{1} \mathbf{H}$ NMR (400 $\left.\mathbf{~ M H z , ~} \mathbf{C D C l}_{3}\right): \delta 8.33-8.31$ (m, 1H), 7.93-7.91 (m, $4 \mathrm{H}), 7.76(\mathrm{~s}, 1 \mathrm{H}), 7.63-7.60(\mathrm{~m}, 3 \mathrm{H}), 7.37-7.28(\mathrm{~m}, 6 \mathrm{H}), 7.16-7.12(\mathrm{~m}, 3 \mathrm{H}), 5.69(\mathrm{~s}, 1 \mathrm{H}), 3.74(\mathrm{~s}$, 3H), 2.47 (s, 3H); ${ }^{13} \mathbf{C}$ NMR(100 MHz, $\left.\mathbf{C D C l}_{3}\right): \delta 179.4,159.4,148.7,143.5,138.7,137.8,134.9$, $132.7,132.1,130.8,129.6,129.5,128.7,128.5,127.9,127.4,127.3,127.2,126.9,125.5,124.9$, 122.6, 120.6, 118.7, 114.5, 114.4, 113.9, 55.2, 21.6; FT-IR (neat, $\mathrm{cm}^{-1}$ ): 3505, 3157, 2766, 1941, 1704, 1593, 1362, 1092, 717; HRMS (ESI-TOF) m/z Calcd for $\mathrm{C}_{31} \mathrm{H}_{26} \mathrm{NO}_{4} \mathrm{~S}[\mathrm{M}+\mathrm{H}]^{+}:$: 508.1583, found 508.1581 .

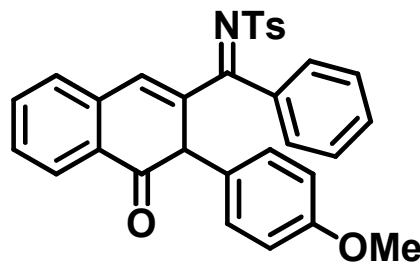

$N$-((3-(2-Methoxyphenyl)-4-oxo-3,4-dihydronaphthalen-2-yl)(phenyl)methylene)-4methylbenzenesulfonamide (6d): Compound $\mathbf{6 d}$ was prepared following the GP-1 using phthalaldehyde (5a, $1 \mathrm{mmol})$ and $N$-((E)-3-(2-methoxyphenyl)-1-phenylallylidene)-4methylbenzenesulfonamide (1d, $1 \mathrm{mmol})$ as starting material and afforded the title compound $\mathbf{6 d}$ (using $14 \%$ ethyl acetate-petroleum ether $(\mathrm{v} / \mathrm{v})$ as an eluent for purification) as pale yellow semi solid, Yield: 69\% (349.0 mg); ${ }^{1} \mathbf{H}$ NMR (400 MHz, $\left.\mathbf{C D C l}_{3}\right): \delta 8.41(\mathrm{~s}, 1 \mathrm{H}), 8.00$ (s, 3H), 7.64-7.62 (m, 2H), 7.40-7.39 (m, 3H), $7.28(\mathrm{~m}, 2 \mathrm{H}), 7.21-7.14(\mathrm{~m}, 3 \mathrm{H}), 7.04(\mathrm{~m}, 2 \mathrm{H}), 6.89(\mathrm{t}, J=7.2 \mathrm{~Hz}, 1 \mathrm{H})$, 6.51(d, $J=8.0 \mathrm{~Hz}, 1 \mathrm{H}), 6.05(\mathrm{~s}, 1 \mathrm{H}), 3.52(\mathrm{~s}, 3 \mathrm{H}), 2.49(\mathrm{~s}, 3 \mathrm{H}) ;{ }^{13} \mathbf{C}$ NMR (100 MHz, CDCl $): \delta$ $180.0,155.3,149.6,143.4,138.9,137.7,135.8,133.1,132.8,132.0,130.1,129.5,129.3,129.1$, 128.7, 128.6, 128.3, 127.3, 127.1, 125.9, 123.4, 122.9, 122.5, 121.2, 116.8, 110.0, 55.2, 21.7; FT-IR $\left(\mathrm{KBr}, \mathrm{cm}^{-1}\right): 3515,3087,2696,1911,1698,1593,1354,1122,987,737$; HRMS (ESI-TOF) $\mathrm{m} / \mathrm{z}$ Calcd for $\mathrm{C}_{31} \mathrm{H}_{26} \mathrm{NO}_{4} \mathrm{~S}[\mathrm{M}+\mathrm{H}]+:$ 508.1583, found 508.1585. 
<smiles></smiles>

$N$-((3-(4-Bromophenyl)-4-oxo-3,4-dihydronaphthalen-2-yl)(phenyl)methylene)-4-

methylbenzenesulfonamide (6e): Compound $\mathbf{6 e}$ was prepared following the GP-1 using phthalaldehyde (5a, $1 \mathrm{mmol})$ and $N$-((E)-3-(4-bromophenyl)-1-phenylallylidene)-4methylbenzenesulfonamide $(\mathbf{1 e}, 1 \mathrm{mmol})$ as starting material and afforded the title compound $\mathbf{6 e}$ (using 16\% ethyl acetate-petroleum ether (v/v) as an eluent for purification) as pale yellow solid, Yield: 82\% (445.0 mg); mp 159-161 ${ }^{\circ} \mathrm{C}$; ${ }^{1} \mathbf{H}$ NMR (400 MHz, $\left.\mathbf{C D C l}_{3}\right): \delta 8.33-8.31(\mathrm{~m}, 1 \mathrm{H}), 7.96-$ $7.90(\mathrm{~m}, 3 \mathrm{H}), 7.81(\mathrm{~s}, 1 \mathrm{H}), 7.66-7.61(\mathrm{~m}, 2 \mathrm{H}), 7.39-7.35(\mathrm{~m}, 5 \mathrm{H}), 7.28-7.26(\mathrm{~m}, 3 \mathrm{H}), 7.18-7.14(\mathrm{~m}$, 3H), 5.55 (s, 1H), 2.48 (s, 3H); ${ }^{13} \mathbf{C}$ NMR (100 MHz, $\left.\mathbf{C D C l}_{3}\right): \delta 179.0,148.4,143.7,138.6,137.6$, $134.3,133.0,132.9,132.8,132.1,129.7,129.6,128.6,128.1,127.7,127.3,127.2$, 125.0, 122.8, 122.6, 121.1, 117.8, 21.7; FT-IR $\left(\mathrm{KBr}, \mathrm{cm}^{-1}\right)$ : 3515, 3126, 2933, 2871, 2178, 1910, 1823, 1712, 1034, 651; HRMS (ESI-TOF) $\mathrm{m} / \mathrm{z}$ Calcd for $\mathrm{C}_{30} \mathrm{H}_{23} \mathrm{BrNO}_{3} \mathrm{~S}[\mathrm{M}+\mathrm{H}]^{+}:$556.0582, found 556.0581(one of the major peaks)<smiles></smiles>

$N$-((3-(4-Chlorophenyl)-4-oxo-3,4-dihydronaphthalen-2-yl)(phenyl)methylene)-4-

methylbenzenesulfonamide (6f): Compound $\mathbf{6 f}$ was prepared following the GP-1 using phthalaldehyde (5a, $1 \mathrm{mmol})$ and $N$-((E)-3-(4-chlorophenyl)-1-phenylallylidene)-4methylbenzenesulfonamide (1f, $1 \mathrm{mmol}$ ) as starting material and afforded the title compound $\mathbf{6 f}$ (using $15 \%$ ethyl acetate-petroleum ether (v/v) as an eluent for purification) as yellowish solid, Yield: 85\% (434.0 mg); mp 168-170 ${ }^{\circ} \mathrm{C}$; ${ }^{1} \mathbf{H}$ NMR (400 $\left.\mathbf{M H z}, \mathbf{C D C l}_{3}\right): \delta 8.30-8.29(\mathrm{~m}, 1 \mathrm{H}), 7.93-$ $7.87(\mathrm{~m}, 4 \mathrm{H}), 7.77(\mathrm{~s}, 1 \mathrm{H}), 7.62-7.60(\mathrm{~m}, 3 \mathrm{H}), 7.35-7.33(\mathrm{~m}, 4 \mathrm{H}), 7.25-7.19(\mathrm{~m}, 1 \mathrm{H}), 7.15-7.13(\mathrm{~m}$, 4H), $5.46(\mathrm{~s}, 1 \mathrm{H}), 2.45(\mathrm{~s}, 3 \mathrm{H}) ;{ }^{13} \mathbf{C}$ NMR (100 MHz, $\left.\mathbf{C D C l}_{3}\right): \delta 178.8,148.4,143.5,138.5,137.5$, $134.4,134.3,132.8,132.7,132.2$, 132.0, 129.5, 129.45, 129.1, 128.5, 127.9, 127.5, 127.2, 127.0, 124.9, 122.5, 121.0, 117.7, 21.5; FT-IR $\left(\mathrm{KBr}, \mathrm{cm}^{-1}\right): 3614,3522,3013,2876,1926,1804,1696$, 1376, 1109, 892; HRMS (ESI-TOF) $\mathrm{m} / \mathrm{z}$ Calcd for $\mathrm{C}_{30} \mathrm{H}_{23} \mathrm{ClNO}_{3} \mathrm{~S}[\mathrm{M}+\mathrm{H}]^{+}:$512.1087, found 512.1091 (one of the major peaks).<smiles></smiles>

$N$-((3-(4-Fluorophenyl)-4-oxo-3,4-dihydronaphthalen-2-yl)(phenyl)methylene)-4methylbenzenesulfonamide (6g): Compound $\mathbf{6 g}$ was prepared following the GP-1 using 
phthalaldehyde (5a, $1 \quad \mathrm{mmol}) \quad$ and $\quad N$-((E)-3-(4-fluorophenyl)-1-phenylallylidene)-4methylbenzenesulfonamide (1g, $1 \mathrm{mmol})$ as starting material and afforded the title compound $\mathbf{6 g}$ (using $16 \%$ ethyl acetate-petroleum ether (v/v) as an eluent for purification) as yellow semi solid, Yield: 88\% (435.0 mg); ${ }^{1} \mathbf{H}$ NMR (400 MHz, $\left.\mathbf{C D C l}_{3}\right): \delta 8.32(\mathrm{~m}, 1 \mathrm{H}), 7.96-7.80(\mathrm{~m}, 4 \mathrm{H}), 7.63(\mathrm{~m}$, 2H), 7.38-7.15 (m, 9H), $6.88(\mathrm{~m}, 2 \mathrm{H}), 5.57(\mathrm{~s}, 1 \mathrm{H}), 2.48(\mathrm{~s}, 3 \mathrm{H}) ;{ }^{13} \mathbf{C}$ NMR (75 MHz, CDCl $): \delta$ 179.1, 164.2 (C-F, $\left.{ }^{1} \mathrm{~J}_{\mathrm{C}-\mathrm{F}}=247.5 \mathrm{~Hz}\right), 160.9\left(\mathrm{C}-\mathrm{F},{ }^{1} \mathrm{~J}_{\mathrm{C}-\mathrm{F}}=247.5 \mathrm{~Hz}\right), 148.6,143.6,138.7,137.7,134.6$, $132.8\left(\mathrm{C}-\mathrm{F},{ }^{3} \mathrm{~J}_{\mathrm{C}-\mathrm{F}}=9.0 \mathrm{~Hz}\right), 132.7\left(\mathrm{C}-\mathrm{F},{ }^{3} \mathrm{~J}_{\mathrm{C}-\mathrm{F}}=9.0 \mathrm{~Hz}\right), 129.6,129.5,129.3,128.6,128.0,127.6$ 127.3, 127.1, 125.0, 122.6, 121.0, 118.0, $116.1\left(\mathrm{C}-\mathrm{F},{ }^{2} \mathrm{~J}_{\mathrm{C}-\mathrm{F}}=21.7 \mathrm{~Hz}\right), 115.9\left(\mathrm{C}-\mathrm{F},{ }^{2} \mathrm{~J}_{\mathrm{C}-\mathrm{F}}=21.7 \mathrm{~Hz}\right)$, 21.6; FT-IR (KBr, $\left.\mathbf{c m}^{-1}\right)$ : 3541, 3126, 2886, 2163, 1914, 1711, 1662, 1336, 1013, 887; HRMS (ESI-TOF) $\mathrm{m} / \mathrm{z}$ Calcd for $\mathrm{C}_{30} \mathrm{H}_{23} \mathrm{FNO}_{3} \mathrm{~S}[\mathrm{M}+\mathrm{H}]^{+}:$496.1383, found 496.1379 .

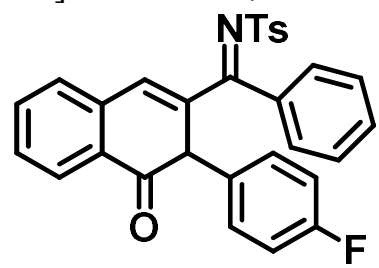

$\mathrm{N}$-((3-(3-Fluorophenyl)-4-oxo-3,4-dihydronaphthalen-2-yl)(phenyl)methylene)-4-

methylbenzenesulfonamide (6h): Compound $\mathbf{6 h}$ was prepared following the GP-1 using phthalaldehyde (5a, $1 \mathrm{mmol})$ and $N$-((E)-3-(3-fluorophenyl)-1-phenylallylidene)-4methylbenzenesulfonamide $(\mathbf{1 h}, 1 \mathrm{mmol})$ as starting material and afforded the title compound $\mathbf{6 h}$ (using 15\% ethyl acetate-petroleum ether $(\mathrm{v} / \mathrm{v})$ as an eluent for purification) as colourless solid, Yield: 78\% (386.0 mg); mp 107-110 ${ }^{\circ} \mathrm{C} ;{ }^{1} \mathbf{H}$ NMR (400 $\left.\mathbf{~ M H z}, \mathbf{C D C l}_{3}\right): \delta 8.34(\mathrm{~s}, 1 \mathrm{H}), 7.95-7.94(\mathrm{~m}$, $3 \mathrm{H}), 7.86(\mathrm{~s}, 1 \mathrm{H}), 7.65(\mathrm{~m}, 2 \mathrm{H}), 7.39-7.34(\mathrm{~m}, 4 \mathrm{H}), 7.26-7.24(\mathrm{~m}, 3 \mathrm{H}), 7.13(\mathrm{~m}, 3 \mathrm{H}), 6.89(\mathrm{~m}, 1 \mathrm{H})$, $5.69(\mathrm{~s}, 1 \mathrm{H}), 2.48(\mathrm{~s}, 3 \mathrm{H}) ;{ }^{13} \mathbf{C}$ NMR (75 MHz, $\left.\mathbf{C D C l}_{3}\right): \delta 178.9,164.4\left(\mathrm{C}-\mathrm{F},{ }^{1} \mathrm{~J}_{\mathrm{C}-\mathrm{F}}=246.7 \mathrm{~Hz}\right)$, $161.1\left(\mathrm{C}-\mathrm{F},{ }^{1} \mathrm{~J}_{\mathrm{C}-\mathrm{F}}=246.7 \mathrm{~Hz}\right), 148.3,143.5,138.5,137.5,136.2\left(\mathrm{C}-\mathrm{F},{ }^{3} \mathrm{~J}_{\mathrm{C}-\mathrm{F}}=8.2 \mathrm{~Hz}\right), 136.1$ $\left(\mathrm{C}-\mathrm{F},{ }^{3} \mathrm{~J}_{\mathrm{C}-\mathrm{F}}=8.2 \mathrm{~Hz}\right), 134.4,132.8,132.7,131.0,130.5\left(\mathrm{C}-\mathrm{F},{ }^{3} \mathrm{~J}_{\mathrm{C}-\mathrm{F}}=9.0 \mathrm{~Hz}\right), 130.4\left(\mathrm{C}-\mathrm{F},{ }^{3} \mathrm{~J}_{\mathrm{C}-\mathrm{F}}=9.0\right.$ Hz), 129.5, 128.5, 127.9, 127.6, 127.2, 127.1, 126.2, 125.0, 122.6, 121.2, 117.9, 117.7, 115.3 (C-F, $\left.{ }^{2} \mathrm{~J}_{\mathrm{C}-\mathrm{F}}=21.0 \mathrm{~Hz}\right), 115.0\left(\mathrm{C}-\mathrm{F},{ }^{2} \mathrm{~J}_{\mathrm{C}-\mathrm{F}}=21.0 \mathrm{~Hz}\right), 21.5$; FT-IR $\left(\mathrm{KBr}, \mathrm{cm}^{-1}\right): 3524,2986,2774,1876$, 1776, 1695, 1564, 1267, 812; HRMS (ESI-TOF) m/z Calcd for $\mathrm{C}_{30} \mathrm{H}_{23} \mathrm{FNO}_{3} \mathrm{~S}[\mathrm{M}+\mathrm{H}]^{+}$: 496.1383, found 496.1380 .<smiles></smiles>

(R)-N-((3-(3,4-Dichlorophenyl)-4-oxo-3,4-dihydronaphthalen-2-yl)(phenyl)methylene)-4-

methylbenzenesulfonamide (6i): Compound $\mathbf{6 i}$ was prepared following the GP-1 using phthalaldehyde (5a, $1 \mathrm{mmol})$ and $N$-((E)-3-(3,4-dichlorophenyl)-1-phenylallylidene)-4methylbenzenesulfonamide (1i, $1 \mathrm{mmol})$ as starting material and afforded the title compound $\mathbf{6 i}$ (using 17\% ethyl acetate-petroleum ether (v/v) as an eluent for purification) as yellow solid, Yield: 91\% (495.0 mg); mp 159-160 ${ }^{\circ} \mathrm{C} ;{ }^{1} \mathbf{H}$ NMR (400 MHz, $\left.\mathbf{C D C l}_{3}\right): \delta 8.31-8.29$ (m, 1H), 7.97-7.93 (m, 4H), $7.86(\mathrm{~s}, 1 \mathrm{H}), 7.65-7.61(\mathrm{~m}, 3 \mathrm{H}), 7.39-7.37(\mathrm{~m}, 3 \mathrm{H}), 7.28-7.14(\mathrm{~m}, 5 \mathrm{H}), 5.62(\mathrm{~s}, 1 \mathrm{H}), 2.48(\mathrm{~s}$, $3 \mathrm{H}) ;{ }^{13} \mathbf{C}$ NMR (100 MHz, $\left.\mathbf{C D C l}_{3}\right): \delta$ 178.8, 148.5, 143.8, 138.3, 137.5, 134.5, 134.2, 133.1, 132.6, 130.6, 129.6, 129.56, 128.7, 128.1, 127.9, 127.3, 125.0, 122.6, 121.4, 116.7, 21.7; FT-IR (KBr, cm $\left.{ }^{1}\right)$ : 3442, 3058, 2921, 1976, 1709, 1552, 1287, 1146, 757; HRMS (ESI-TOF) $\mathrm{m} / \mathrm{z}$ Calcd for $\mathrm{C}_{30} \mathrm{H}_{22} \mathrm{Cl}_{2} \mathrm{NO}_{3} \mathrm{~S}[\mathrm{M}+\mathrm{H}]^{+}: 546.0697$, found 546.0699 (one of the major peaks). 
<smiles></smiles>

$N$-((3-(4-Cyanophenyl)-4-oxo-3,4-dihydronaphthalen-2-yl)(phenyl)methylene)-4-

methylbenzenesulfonamide $(\mathbf{6 j})$ : Compound $\mathbf{6 j}$ was prepared following the GP-1 using phthalaldehyde (5a, $1 \mathrm{mmol})$ and $N$-((E)-3-(4-cyanophenyl)-1-phenylallylidene)-4methylbenzenesulfonamide $(\mathbf{1 j}, 1 \mathbf{m m o l})$ as starting material and afforded the title compound $\mathbf{6 j}$ (using 16\% ethyl acetate-petroleum ether (v/v) as an eluent for purification) as white solid, Yield: 83\% (416.0 mg); mp 164-165 ${ }^{\circ} \mathrm{C}$; ${ }^{1} \mathbf{H}$ NMR (400 MHz, $\left.\mathbf{C D C l}_{3}\right): \delta$ 8.29-8.27 (m, 1H), 8.27-7.81 (m, 4H), 7.63-7.60 (m, 2H), 7.41-7.34 (m, 7H), 7.24-7.11 (m, 4H), 5.56 (s, 1H), $2.46(\mathrm{~s}, 3 \mathrm{H})$; ${ }^{13} \mathbf{C}$ NMR (100 MHz, $\left.\mathbf{C D C l}_{3}\right): \delta 178.8,148.5,144.0,139.6,138.6,137.5,134.1,133.3,132.5,131.7,129.8$, 128.3, 128.1, 127.6, 127.4, 125.3, 122.7, 121.9, 118.4, 117.7, 112.0, 21.8; FT-IR $\left(\mathrm{KBr}, \mathrm{cm}^{-1}\right): 3441$, 3216, 2756, 2421, 2112, 1914, 1681, 1542, 1436, 1133, 967; HRMS (ESI-TOF) m/z Calcd for $\mathrm{C}_{31} \mathrm{H}_{23} \mathrm{~N}_{2} \mathrm{O}_{3} \mathrm{~S}[\mathrm{M}+\mathrm{H}]^{+}:$503.1429, found 503.1426.

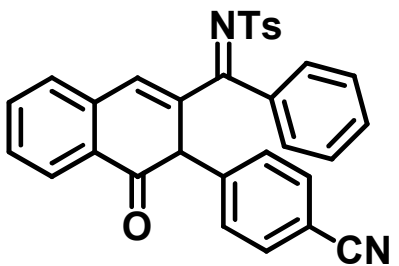

\section{4-Methyl- $N$-((4-oxo-3-phenyl-3,4-dihydronaphthalen-2-yl)(p-}

tolyl)methylene)benzenesulfonamide (6k): Compound $\mathbf{6 k}$ was prepared following the GP-1 using phthalaldehyde (5a, 1 mmol) and 4-methyl-N- $((E)-3-p h e n y l-1-(p$ tolyl)allylidene)benzenesulfonamide $(1 \mathbf{k}, 1 \mathrm{mmol})$ as starting material and afforded the title compound $\mathbf{6 k}$ (using 14\% ethyl acetate-petroleum ether (v/v) as an eluent for purification) as light brown solid, Yield: $67 \%$ (328.0 mg); mp 129-130 ${ }^{\circ} \mathrm{C} ;{ }^{1} \mathbf{H}$ NMR (400 MHz, $\left.\mathbf{C D C l}_{3}\right): \delta 8.36-8.34(\mathrm{~m}$, $1 \mathrm{H}), 7.92-7.90(\mathrm{~m}, 3 \mathrm{H}), 7.75(\mathrm{~s}, 1 \mathrm{H}), 7.63-7.61(\mathrm{~m}, 3 \mathrm{H}), 7.36-7.08(\mathrm{~m}, 8 \mathrm{H}), 6.95-6.93(\mathrm{~m}, 2 \mathrm{H}), 5.73$ (s, 1H), 2.47 (s, 3H), 2.29 (s, 3H); ${ }^{13} \mathbf{C}$ NMR(100 MHz, $\left.\mathbf{C D C l}_{3}\right): \delta 178.8,148.4,143.8,143.4,138.9$, $135.0,133.8,132.8,130.7,129.9,129.5,129.0,128.7,128.5,128.3,127.3,127.3,127.0,126.9$, 126.5, 124.9, 122.7, 120.7, 119.2, 21.6; FT-IR $\left(\mathrm{KBr}, \mathrm{cm}^{-1}\right): 3524,3410,3298,2913,2293,2013$, 1901, 1728, 1687, 1210, 709; HRMS (ESI-TOF) m/z Calcd for $\mathrm{C}_{31} \mathrm{H}_{26} \mathrm{NO}_{3} \mathrm{~S}[\mathrm{M}+\mathrm{H}]^{+}:$492.1633, found 492.1635 .<smiles>Cc1ccc(C(=[NH2+])C2=Cc3ccccc3C(=O)C2c2ccccc2)cc1</smiles>

$\mathrm{N}$-((4-Bromophenyl)(4-oxo-3-phenyl-3,4-dihydronaphthalen-2-yl)methylene)-4-

methylbenzenesulfonamide (61): Compound $\mathbf{6 1}$ was prepared following the GP-1 using phthalaldehyde (5a, $1 \mathrm{mmol})$ and $N$-((E)-1-(4-bromophenyl)-3-phenylallylidene)-4- 
methylbenzenesulfonamide (11, $1 \mathrm{mmol})$ as starting material and afforded the title compound $\mathbf{6 1}$ (using 16\% ethyl acetate-petroleum ether (v/v) as an eluent for purification) as pale yellow solid;

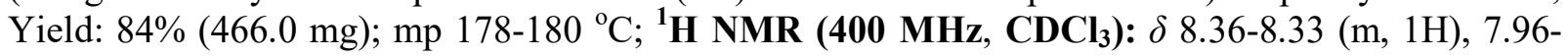
$7.91(\mathrm{~m}, 3 \mathrm{H}), 7.80(\mathrm{~s}, 1 \mathrm{H}), 7.65-7.62(\mathrm{~m}, 3 \mathrm{H}), 7.38-7.36(\mathrm{~m}, 3 \mathrm{H}), 7.28-7.24(\mathrm{~m}, 5 \mathrm{H}), 7.12-7.10(\mathrm{~m}$, 2H), 5.74 (s, 1H), 2.47 (s, 3H); ${ }^{13} \mathbf{C}$ NMR (100 MHz, $\left.\mathbf{C D C l}_{3}\right): \delta$ 178.1, 148.6, 143.7, 138.4, 136.7, $134.1,133.7,132.8,131.2,131.0,129.6,129.2$, 128.6, 128.5, 127.8, 127.6, 127.4, 127.2, 125.1, 122.7, 121.1, 21.7; FT-IR (KBr, cm $\left.{ }^{-1}\right): 3387,3123,2879,1896,1698,1542,1223,1165,987,897$; HRMS (ESI-TOF) $\mathrm{m} / \mathrm{z}$ Calcd for $\mathrm{C}_{30} \mathrm{H}_{23} \mathrm{BrNO}_{3} \mathrm{~S}[\mathrm{M}+\mathrm{H}]^{+}: 556.0582$, found 556.0584 (one of the major peaks).

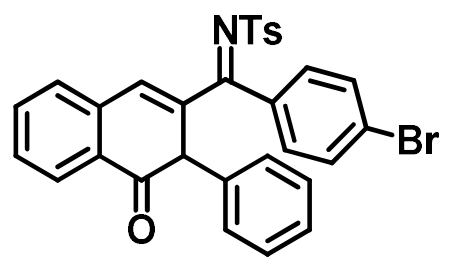

\title{
4-Methyl- $N$-((3-nitrophenyl)(4-oxo-3-phenyl-3,4-dihydronaphthalen-2-
}

yl)methylene)benzenesulfonamide $(6 \mathrm{~m})$ : Compound $\mathbf{6 m}$ was prepared following the GP-1 using phthalaldehyde (5a, $1 \mathrm{mmol})$ and 4-methyl- $N$-((E)-1-(3-nitrophenyl)-3phenylallylidene)benzenesulfonamide $(\mathbf{1} \mathbf{m}, 1 \mathrm{mmol})$ as starting material and afforded the title compound $\mathbf{6 m}$ (using $18 \%$ ethyl acetate-petroleum ether $(\mathrm{v} / \mathrm{v})$ as an eluent for purification) as viscous liquid, Yield: 74\% (386.0 mg); ${ }^{1} \mathbf{H}$ NMR (400 MHz, $\left.\mathbf{C D C l}_{3}\right): \delta 8.36-8.34$ (m, 1H), 8.14-8.12 (m, 1H), 7.98-7.92 (m, 5H), 7.67-7.65 (m, 2H), 7.54-7.52 (m, 1H), 7.41-7.39 (m, 3H), 7.31-7.28 (m, 2H), 7.27-7.17 (m, 3H), $5.80(\mathrm{~s}, 1 \mathrm{H}), 2.49$ (s, 3H); ${ }^{13} \mathbf{C}$ NMR(100 MHz, CDCl $\left.\mathbf{~ M}_{3}\right): \delta 177.0,148.8$, $147.7,144.1,139.5,138.0,134.8,133.7,133.4,132.8,129.7,128.9,128.7,128.6,127.8,127.5$, 126.5, 125.4, 123.9, 122.8, 121.9, 21.7; FT-IR (neat, $\mathrm{cm}^{-1}$ ): 3604, 3512, 3413, 3163, 2966, 1856, 1784, 1688, 1576, 1309, 1092, 758; HRMS (ESI-TOF) $\mathrm{m} / \mathrm{z}$ Calcd for $\mathrm{C}_{30} \mathrm{H}_{23} \mathrm{~N}_{2} \mathrm{O}_{5} \mathrm{~S}[\mathrm{M}+\mathrm{H}]^{+}$: 523.1328 , found 523.1324 .

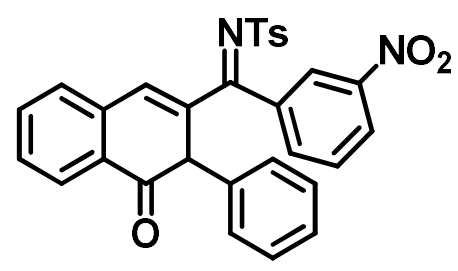

\begin{abstract}
4-Methyl- $N$-((4-0xo-3-(p-tolyl)-3,4-dihydronaphthalen-2-yl)(p-
tolyl)methylene)benzenesulfonamide (6n): Compound 6n was prepared following the GP-1 using phthalaldehyde (5a, $1 \mathrm{mmol})$ and $N$-((E)-1,3-di-p-tolylallylidene)-4-methylbenzenesulfonamide (1n, $1 \mathrm{mmol}$ ) as starting material and afforded the title compound 6n (using 15\% ethyl acetate-petroleum ether (v/v) as an eluent for purification) as fade brown solid, Yield: $71 \%(358.0 \mathrm{mg}) ; \mathrm{mp} 152-153{ }^{\circ} \mathrm{C}$; ${ }^{1}$ H NMR (400 MHz, CDCl $\left.\mathbf{~ M}_{3}\right): \delta 8.35-8.32(\mathrm{~m}, 1 \mathrm{H}), 7.97-7.89(\mathrm{~m}, 3 \mathrm{H}), 7.69(\mathrm{~s}, 1 \mathrm{H}), 7.62-7.50(\mathrm{~m}$, 2H), 7.35-7.33 (m, 3H), 7.28-7.24 (m, 3H), $7.03(\mathrm{~m}, 2 \mathrm{H}), 6.96-6.94(\mathrm{~m}, 2 \mathrm{H}), 5.72(\mathrm{~s}, 1 \mathrm{H}), 2.46(\mathrm{~s}$, 3H), 2.30 (s, 3H), 2.27 (s, 3H); ${ }^{13} \mathbf{C}$ NMR(100 MHz, $\left.\mathbf{C D C l}_{3}\right): \delta 178.8,148.5,143.8,143.3,138.9$, $138.1,135.0,132.7,130.6,130.0,129.7,129.4,128.7,128.4,127.3,127.2,126.7,126.5,124.8$, 122.7, 121.1, 120.4, 119.2, 21.63, 21.6, 21.2; FT-IR $\left(\mathrm{KBr}, \mathrm{cm}^{-1}\right): 3454,3354,3189,2875,2239$, 2021, 1897, 1788, 1710, 1287, 908; HRMS (ESI-TOF) m/z Calcd for $\mathrm{C}_{32} \mathrm{H}_{28} \mathrm{NO}_{3} \mathrm{~S}[\mathrm{M}+\mathrm{H}]^{+}$:
\end{abstract}


506.1790, found 506.1785.<smiles>Cc1ccc(C(=[N+]=[N-])C2=Cc3ccccc3C(=O)C2c2ccc(C)cc2)cc1</smiles>

N-((4-Bromophenyl)(4-oxo-3-(p-tolyl)-3,4-dihydronaphthalen-2-yl)methylene)-4methylbenzenesulfonamide (6o): Compound $\mathbf{6 0}$ was prepared following the GP-1 using phthalaldehyde (5a, $1 \mathrm{mmol})$ and $N$-((E)-1-(4-bromophenyl)-3-( $p$-tolyl)allylidene)-4methylbenzenesulfonamide $(\mathbf{1 0}, 1 \mathrm{mmol})$ as starting material and afforded the title compound $\mathbf{6 0}$ (using 16\% ethyl acetate-petroleum ether (v/v) as an eluent for purification) as yellowish semi solid, Yield: 79\% (449.0 mg); ${ }^{1} \mathbf{H}$ NMR (400 MHz, $\left.\mathbf{C D C l}_{3}\right): \delta 8.32(\mathrm{~m}, 1 \mathrm{H}), 7.92-7.89(\mathrm{~m}, 3 \mathrm{H}), 7.76(\mathrm{~s}$, $1 \mathrm{H}), 7.66-7.61(\mathrm{~m}, 2 \mathrm{H}), 7.37-7.35(\mathrm{~m}, 2 \mathrm{H}), 7.28-7.26(\mathrm{~m}, 3 \mathrm{H}), 7.16-7.14(\mathrm{~m}, 3 \mathrm{H}), 7.05(\mathrm{~m}, 2 \mathrm{H}), 5.30$ $(\mathrm{s}, 1 \mathrm{H}), 2.47$ (s, 3H), $2.28(\mathrm{~s}, 3 \mathrm{H}) ;{ }^{13} \mathbf{C}$ NMR (100 MHz, $\left.\mathbf{C D C l}_{3}\right): \delta$ 178.0, 148.7, 145.6, 143.7, $138.5,138.4,136.7,134.2,132.7,132.0,131.9,131.2,131.0,130.5,130.0,129.9,129.8,129.5$, 128.6, 128.5, 127.4, 127.0, 125.0, 122.7, 120.8, 120.5, 21.6, 21.2; FT-IR $\left(\mathrm{KBr}, \mathrm{cm}^{-1}\right)$ : 3617, 3554, 3352, 3273, 2853, 2139, 1981, 1849, 1718, 1632, 1148, 851; HRMS (ESI-TOF) $\mathrm{m} / \mathrm{z}$ Calcd for $\mathrm{C}_{31} \mathrm{H}_{25} \mathrm{BrNO}_{3} \mathrm{~S}[\mathrm{M}+\mathrm{H}]^{+}: 570.0739$, found 570.0736 (one of the major peaks).<smiles></smiles>

(R)- $N$-((4-Bromophenyl)(3-(4-fluorophenyl)-4-oxo-3,4-dihydronaphthalen-2-yl)methylene)-4methylbenzenesulfonamide (6p): Compound $\mathbf{6 p}$ was prepared following the GP-1 using phthalaldehyde (5a, $1 \mathrm{mmol})$ and $N$-((E)-1-(4-bromophenyl)-3-(4-fluorophenyl)allylidene)-4methylbenzenesulfonamide $(\mathbf{1 p}, 1 \mathrm{mmol})$ as starting material and afforded the title compound $\mathbf{6 p}$ (using 19\% ethyl acetate-petroleum ether (v/v) as an eluent for purification) as pale yellow solid, Yield: 88\% (504.0 mg); $\mathrm{mp} 173-175{ }^{\circ} \mathrm{C} ;{ }^{1} \mathbf{H}$ NMR (400 $\left.\mathbf{~ M H z}, \mathbf{C D C l}_{3}\right): \delta 8.33-8.31(\mathrm{~m}, 1 \mathrm{H}), 7.95-$ $7.89(\mathrm{~m}, 3 \mathrm{H}), 7.79(\mathrm{~s}, 1 \mathrm{H}), 7.64-7.62(\mathrm{~m}, 2 \mathrm{H}), 7.38-7.28(\mathrm{~m}, 3 \mathrm{H}), 7.14-7.12(\mathrm{~m}, 3 \mathrm{H}), 7.03(\mathrm{~m}, 2 \mathrm{H})$, $6.92(\mathrm{~m}, 2 \mathrm{H}), 5.70(\mathrm{~s}, 1 \mathrm{H}), 2.47(\mathrm{~s}, 3 \mathrm{H}) ;{ }^{13} \mathbf{C ~ N M R}\left(\mathbf{1 0 0} \mathbf{M H z}, \mathbf{C D C l}_{3}\right): \delta 177.9,163.7\left(\mathrm{C}-\mathrm{F},{ }^{1} \mathrm{~J}_{\mathrm{C}-\mathrm{F}}=\right.$ $247.0 \mathrm{~Hz}), 161.2\left(\mathrm{C}-\mathrm{F},{ }^{1} \mathrm{~J}_{\mathrm{C}-\mathrm{F}}=247.0 \mathrm{~Hz}\right), 148.6,143.7,138.2,136.4,134.0,132.7,131.2,130.8$, 129.7, 129.5, 128.5, 128.0, 127.5, $127.2\left(\mathrm{C}-\mathrm{F},{ }^{3} \mathrm{~J}_{\mathrm{C}-\mathrm{F}}=8.0 \mathrm{~Hz}\right), 127.1\left(\mathrm{C}-\mathrm{F},{ }^{3} \mathrm{~J}_{\mathrm{C}-\mathrm{F}}=8.0 \mathrm{~Hz}\right), 125.0$, 122.5, 120.9, 117.7, $116.1\left(\mathrm{C}-\mathrm{F},{ }^{2} \mathrm{~J}_{\mathrm{C}-\mathrm{F}}=20.0 \mathrm{~Hz}\right), 115.9\left(\mathrm{C}-\mathrm{F},{ }^{2} \mathrm{~J}_{\mathrm{C}-\mathrm{F}}=20.0 \mathrm{~Hz}\right), 21.5$; FT-IR $(\mathrm{KBr}$, $\mathrm{cm}^{-1}$ ): 3512, 3051, 2876, 2788, 1826, 1704, 1682, 1498, 1264, 752; HRMS (ESI-TOF) m/z Calcd for $\mathrm{C}_{30} \mathrm{H}_{22} \mathrm{BrFNO}_{3} \mathrm{~S}[\mathrm{M}+\mathrm{H}]^{+}: 574.0488$, found 574.0491 (one of the major peaks).<smiles>[NH2+]=C(C1=Cc2ccccc2C(=O)C1c1ccc(F)cc1)c1ccc(Br)cc1</smiles>

$\mathrm{N}$-((3-(4-Chlorophenyl)-4-oxo-3,4-dihydronaphthalen-2-yl)(p-tolyl)methylene)-4-

methylbenzenesulfonamide (6q): Compound $\mathbf{6 q}$ was prepared following the GP-1 using 
phthalaldehyde (5a, $1 \mathrm{mmol}) \quad$ and $\quad N-((E)-3-(4-c h l o r o p h e n y l)-1-(p-t o l y l) a l l y l i d e n e)-4-$ methylbenzenesulfonamide (1q, $1 \mathrm{mmol})$ as starting material and afforded the title compound $\mathbf{6 q}$ (using $15 \%$ ethyl acetate-petroleum ether $(\mathrm{v} / \mathrm{v})$ as an eluent for purification) as colourless thick liquid, Yield: 83\% (435.0 mg); ${ }^{1} \mathbf{H}$ NMR (400 MHz, $\left.\mathbf{C D C l}_{3}\right): \delta 8.33-8.31(\mathrm{~m}, 1 \mathrm{H}), 7.93-7.88$ (m, $3 \mathrm{H}), 7.73(\mathrm{~s}, 1 \mathrm{H}), 7.64-7.60(\mathrm{~m}, 2 \mathrm{H}), 7.37-7.35(\mathrm{~m}, 3 \mathrm{H}), 7.28-7.22(\mathrm{~m}, 5 \mathrm{H}), 6.98-6.96(\mathrm{~m}, 2 \mathrm{H}), 5.63$ $(\mathrm{s}, 1 \mathrm{H}), 2.47(\mathrm{~s}, 3 \mathrm{H}), 2.31(\mathrm{~s}, 3 \mathrm{H}),{ }^{13} \mathbf{C}$ NMR (100 MHz, $\left.\mathbf{C D C l}_{3}\right): \delta 178.6,148.5,144.2,143.5$, $138.7,134.8,134.4,132.9,132.4,132.2,129.9,129.5,129.1,128.9,128.5,127.3,127.0,124.9$, 122.6, 120.8, 118.0, 21.7; FT-IR (neat, $\mathrm{cm}^{-1}$ ): 3456, 3243, 3015, 2870, 2239, 1831, 1713, 1655, 1121, 798; HRMS (ESI-TOF) $\mathrm{m} / \mathrm{z}$ Calcd for $\mathrm{C}_{31} \mathrm{H}_{25} \mathrm{ClNO}_{3} \mathrm{~S}[\mathrm{M}+\mathrm{H}]^{+}:$:526.1244, found 526.1242 (one of the major peaks)<smiles>Cc1ccc(C(=[NH2+])C2=Cc3ccccc3C(=O)C2c2ccc(Cl)cc2)cc1</smiles>

N-((4-Bromophenyl)(3-(furan-2-yl)-4-oxo-3,4-dihydronaphthalen-2-yl)methylene)-4methylbenzenesulfonamide (6r): Compound $\mathbf{6 r}$ was prepared following the GP-1 using phthalaldehyde (5a, $1 \mathrm{mmol})$ and $N$-((E)-1-(4-bromophenyl)-3-(furan-2-yl)allylidene)-4methylbenzenesulfonamide $(\mathbf{1 r}, 1 \mathrm{mmol})$ as starting material and afforded the title compound $\mathbf{6 r}$ (using $15 \%$ ethyl acetate-petroleum ether $(\mathrm{v} / \mathrm{v})$ as an eluent for purification) as brownish viscous liquid, Yield: 62\% (337.0 mg); ${ }^{1} \mathbf{H}$ NMR (400 MHz, $\left.\mathbf{C D C l}_{3}\right): \delta 8.38-8.35(\mathrm{~m}, 1 \mathrm{H}), 7.83-7.80(\mathrm{~m}$, 2H), $7.75(\mathrm{~d}, J=8.4 \mathrm{~Hz}, 2 \mathrm{H}), 7.63-7.59(\mathrm{~m}, 3 \mathrm{H}), 7.50(\mathrm{~s}, 1 \mathrm{H}), 7.41-7.34(\mathrm{~m}, 4 \mathrm{H}), 7.19(\mathrm{~d}, J=8.0$ $\mathrm{Hz}, 2 \mathrm{H}), 6.40(\mathrm{~d}, J=3.2 \mathrm{~Hz}, 1 \mathrm{H}), 6.32-6.31(\mathrm{~m}, 1 \mathrm{H}), 2.38(\mathrm{~s}, 3 \mathrm{H}),{ }^{13} \mathbf{C}$ NMR (100 MHz, CDCl $\left.\mathbf{3}\right): \delta$ $176.5,149.8,143.7,142.1,135.8,132.7,131.6,131.1,129.7,129.3,128.3,128.1,127.7,127.2$, 125.4, 122.9, 121.2, 112.0, 110.8, 21.6; FT-IR (neat, $\mathrm{cm}^{-1}$ ): 3531, 3326, 2576, 2301, 2132, 2089, 1876, 1711, 1376, 1233, 1037; HRMS (ESI-TOF) m/z Calcd for $\mathrm{C}_{28} \mathrm{H}_{21} \mathrm{BrNO}_{4} \mathrm{~S}[\mathrm{M}+\mathrm{H}]^{+}:$546.0375, found 546.0377 (one of the major peaks).<smiles>[NH2+]=C(C1=Cc2ccccc2C(=O)C1c1ccco1)c1ccc(Br)cc1</smiles>

$N$-((4-Bromophenyl)(4-oxo-3-(thiophen-2-yl)-3,4-dihydronaphthalen-2-yl)methylene)-4methylbenzenesulfonamide (6s): Compound $\mathbf{6 s}$ was prepared following the GP-1 using phthalaldehyde (5a, $1 \mathrm{mmol})$ and $N$-((E)-1-(4-bromophenyl)-3-(thiophen-2-yl)allylidene)-4methylbenzenesulfonamide $(\mathbf{1 s}, 1 \mathrm{mmol})$ as starting material and afforded the title compound $\mathbf{6 s}$ (using 14\% ethyl acetate-petroleum ether (v/v) as an eluent for purification) as Pale yellow semi solid, Yield: 68\% (381.0 mg); ${ }^{1} \mathbf{H}$ NMR (400 MHz, $\left.\mathbf{C D C l}_{3}\right): \delta 8.34-8.33(\mathrm{~m}, 1 \mathrm{H}), 7.92-7.70(\mathrm{~m}, 3 \mathrm{H})$, 7.64-7.62 (m, 3H), 7.35-7.24 (m, 6H), $7.02(\mathrm{~d}, J=8.0 \mathrm{~Hz}, 2 \mathrm{H}), 6.95-6.93(\mathrm{~m}, 1 \mathrm{H}), 6.18(\mathrm{~s}, 1 \mathrm{H}), 2.45$ $(\mathrm{s}, 3 \mathrm{H}) ;{ }^{13} \mathbf{C N M R}\left(\mathbf{1 0 0} \mathbf{~ M H z}, \mathbf{C D C l}_{3}\right): \delta 178.7,148.4,143.9,139.6,138.5,137.5,134.0,133.2$, $132.5,131.7,129.7,128.8,128.2,128.1,127.5,127.3,127.1,125.2,122.6,121.8,118.4,117.7$, 112.0, 21.7; FT-IR (KBr, cm $\left.{ }^{-1}\right): 3123,3086,2486,1826,1691,1513,1353,1267,878,567$; HRMS (ESI-TOF) $\mathrm{m} / \mathrm{z}$ Calcd for $\mathrm{C}_{28} \mathrm{H}_{21} \mathrm{BrNO}_{3} \mathrm{~S}_{2}[\mathrm{M}+\mathrm{H}]^{+}:$562.0146, found 562.0148.(one of the major peaks)

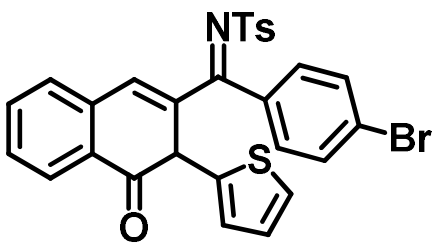


$N$-((3-(4-Chlorophenyl)-6-methoxy-4-oxo-3,4-dihydronaphthalen-2-yl)(phenyl)methylene)-4-

methylbenzenesulfonamide (6t): Compound 6t was prepared following the GP-1 using 4-methoxyphthalaldehyde (5b, $1 \mathrm{mmol})$ and $N$-((E)-3-(4-Chlorophenyl)-1-phenylallylidene)-4methylbenzenesulfonamide (1t, $1 \mathrm{mmol})$ as starting material and afforded the title compound $\mathbf{6 t}$ (using 20\% ethyl acetate-petroleum ether (v/v) as an eluent for purification) as pale yellow solid, Yield: 75\% (405.0 mg); mp 92- $94{ }^{\circ} \mathrm{C}$; ${ }^{1} \mathbf{H}$ NMR (300 $\mathbf{M H z}, \mathbf{C D C l}_{3}$ ): $\delta 7.90-7.87$ (m, 3H), 7.75 (s, $5 \mathrm{H}), 7.54(\mathrm{~d}, J=1.8 \mathrm{~Hz}, \mathrm{~m}, 1 \mathrm{H}), 7.36-7.12(\mathrm{~m}, 12 \mathrm{H}), 5.47(\mathrm{~s}, 1 \mathrm{H}), 3.97(\mathrm{~s}, 3 \mathrm{H}), 2.46(\mathrm{~s}, 3 \mathrm{H}) ;{ }^{13} \mathbf{C}$ NMR (75 MHz, $\left.\mathbf{C D C l}_{3}\right): \delta$ 179.4, 159.0, 147.4, 143.5, 137.9, 134.4, 132.8, 132.6, 132.1, 130.3, 129.6, 129.5, 129.1, 128.2, 128.0, 127.3, 126.3, 121.3, 120.4, 100.9, 55.6, 21.6; FT-IR (KBr, cm $\left.{ }^{-1}\right)$ : 3072, 2977, 2816, 2747, 1672, 1564, 1245, 989, 751; HRMS (ESI-TOF) $\mathrm{m} / \mathrm{z}$ Calcd for $\mathrm{C}_{31} \mathrm{H}_{25} \mathrm{ClNO}_{4} \mathrm{~S}[\mathrm{M}+\mathrm{H}]^{+}: 542.1193$, found 542.1191 (one of the major peaks)<smiles>COc1ccc2c(c1)C(=O)C(c1ccc(Cl)cc1)C(C(=[NH2+])c1ccccc1)=C2</smiles>

\section{Section III:}

Table S1. Optimization of the reaction conditions for isoquinoline synthesis ${ }^{\text {a,b }}$

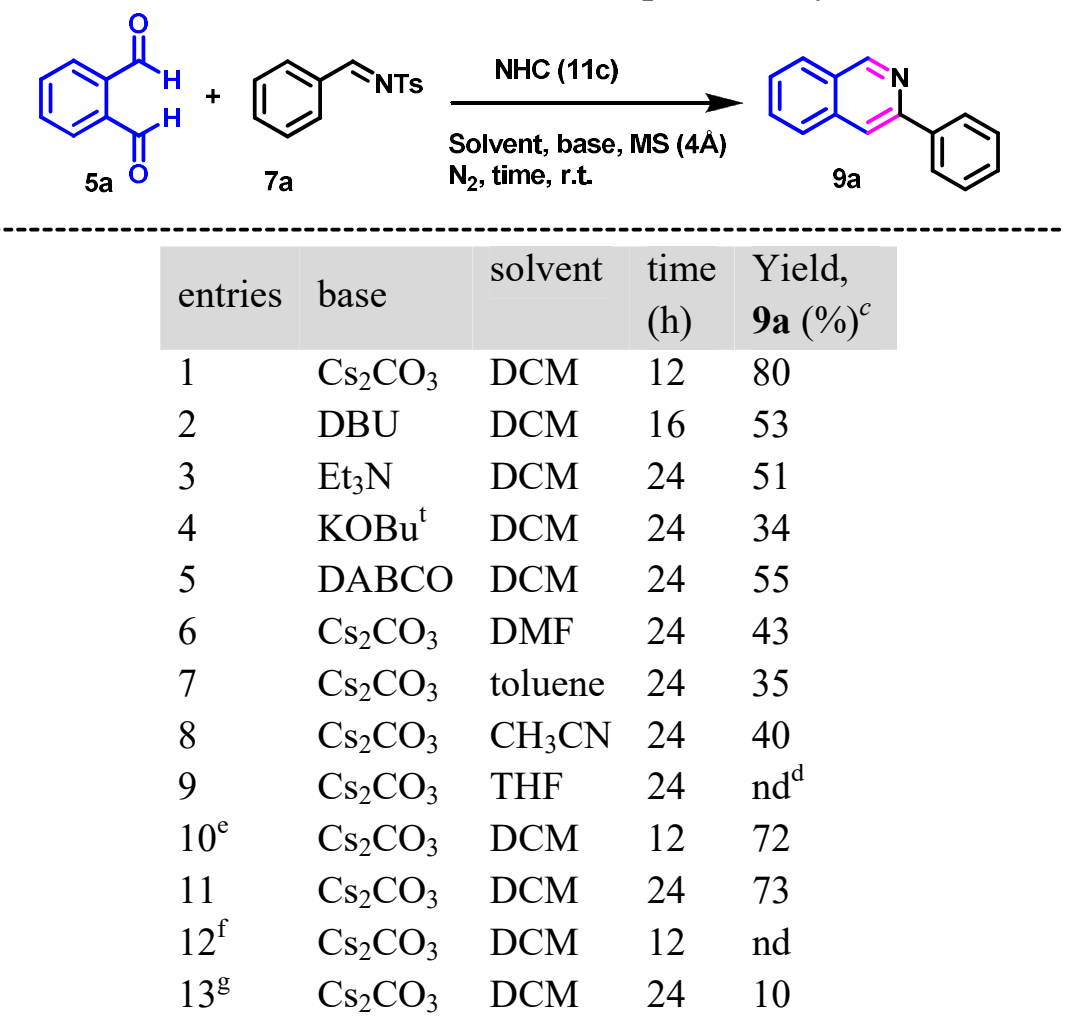

${ }^{a}$ Reaction conditions: Phthalaldehyde $(5 \mathbf{a}, 1.0 \mathrm{mmol})$, imine $(7 \mathbf{a}, 1.2 \mathrm{mmol})$, solvent $(5 \mathrm{~mL})$, NHC precursor 11c $(1.15 \mathrm{mmol})$, base $(1.2 \mathrm{mmol})$, stirred at ambient temperature. ${ }^{\mathrm{b}} \mathrm{MS}$ : Molecular sieves. ${ }^{c}$ Yield of the product obtained after purification by silica gel column chromatography. ${ }^{\mathrm{d}} \mathbf{9} \mathbf{a}$ not detected. base: 1.0 mmol. ${ }^{\mathrm{f}}$ Without NHC. ${ }^{\mathrm{g}} \mathrm{NHC}$ : $15 \mathrm{~mol} \%$; base: $20 \mathrm{~mol} \%$. 
General procedure for the synthesis of 3-aryl substituted isoquinolines (GP-2): Under nitrogen atomsphere, phthalaldehyde $(\mathbf{5 a}, 1.0 \mathrm{mmol})$ and $N$-sulfinyl aldimines ${ }^{2}(\mathbf{7}, 1.2 \mathrm{mmol})$ were added in dry $\mathrm{CH}_{2} \mathrm{Cl}_{2}(5 \mathrm{~mL})$ in the presence of thiazolium bromide $11 \mathrm{c}(1.15 \mathrm{mmol})$. Activated MS- $4 \AA$ ( $\left.300 \mathrm{mg}\right)$ and $\mathrm{Cs}_{2} \mathrm{CO}_{3}(1.2 \mathrm{mmol})$ were added to the reaction mixture and stirred at ambient temperature for 10-16 $\mathrm{h}$. Upon completion (monitored through TLC), the reaction mixture was filtered through the cellite bed and the filtrate was extracted with $\mathrm{CH}_{2} \mathrm{Cl}_{2}(2 \times 15 \mathrm{~mL})$. The combined organic layer was washed with water $(3 \times 10 \mathrm{~mL})$ and dried over anhydrous $\mathrm{Na}_{2} \mathrm{SO}_{4}$, filtered and evaporated in a rotary evaporator under reduced pressure. The residue was chromatographed on a silica gel column (60-120 mesh) using ethyl acetate-petroleum ether $(5 \%$ to $15 \%, \mathrm{v} / \mathrm{v})$ as an eluent, which afforded the corresponding 3-aryl substituted isoquinoline (9).

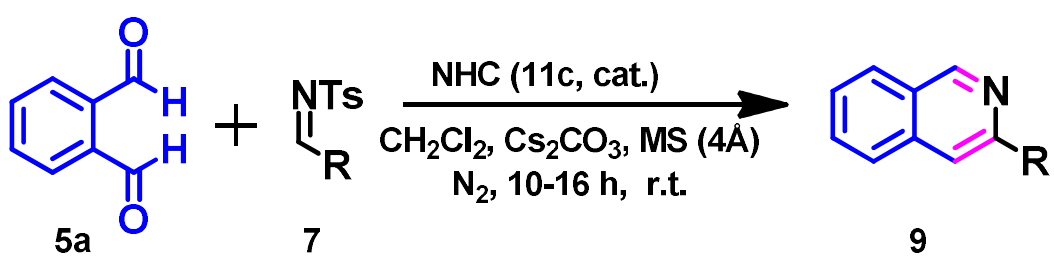

\section{Section IIIa. Characterization data of compound 9a-q:}

3-Phenylisoquinoline (9a): ${ }^{3}$ Compound 9a, was prepared following the GP-2 using phthalaldehyde (5a, $1 \mathrm{mmol})$ and $N$-benzylidene-4-methylbenzenesulfonamide (7a, $1.2 \mathrm{mmol}$ ) as starting material and afforded the title compound 9a (using 5\% ethyl acetate-petroleum ether (v/v) as an eluent for purification) as white solid, Yield: $80 \%(164.0 \mathrm{mg})$; $\mathrm{mp} 85{ }^{\circ} \mathrm{C}\left(\right.$ lit. $\left.^{3} 82-84{ }^{\circ} \mathrm{C}\right) ;{ }^{1} \mathbf{H}$ NMR (400 MHz, $\mathbf{C D C l}$ ): $\delta 9.35$ (s, $1 \mathrm{H}), 8.13-8.07(\mathrm{~m}, 3 \mathrm{H}), 8.00(\mathrm{~d}, J=8 \mathrm{~Hz}, 1 \mathrm{H}), 7.88(\mathrm{~d}, J=8 \mathrm{~Hz}, 1 \mathrm{H}), 7.72-7.69(\mathrm{~m}, 1 \mathrm{H}), 7.61-7.57(\mathrm{~m}$, 1H), 7.53-7.49 (m, 2H), 7.44-7.40(m, 1H); ${ }^{13} \mathbf{C}$ NMR (100 MHz, $\left.\mathbf{C D C l}_{3}\right): \delta 152.5,151.4,139.7,136.9$, $130.7,129.0,128.7,127.9,127.8,127.3,127.2,127.1,116.7$.

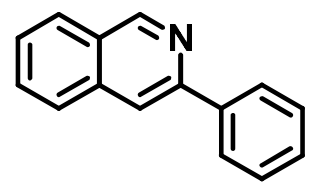

3-(4-Chlorophenyl)isoquinoline $(9 \mathbf{b}):^{3}$ Compound 9b, was prepared following the GP-2 using phthalaldehyde (5a, $1 \mathrm{mmol}$ ) and $N$-(4-chlorobenzylidene)-4-methylbenzenesulfonamide (7b, $1.2 \mathrm{mmol}$ ) as starting material and afforded the title compound $\mathbf{9 b}$ (using 7\% ethyl acetate-petroleum ether (v/v) as an eluent for purification) as white solid, Yield: $75 \%(180 \mathrm{mg}) ; \mathrm{mp} 129-130{ }^{\circ} \mathrm{C}$ (lit. $\left.{ }^{3} 132-134{ }^{\circ} \mathrm{C}\right) ;{ }^{1} \mathbf{H}$ NMR (400 MHz, CDCl $): \delta 9.32(\mathrm{~s}, 1 \mathrm{H}), 8.07-7.98(\mathrm{~m}, 4 \mathrm{H}), 7.86(\mathrm{~d}, J=8 \mathrm{~Hz}, 1 \mathrm{H}), 7.70(\mathrm{t}, J=7.2 \mathrm{~Hz}$, 1H), $7.59(\mathrm{t}, J=7.2 \mathrm{~Hz}, 1 \mathrm{H}), 7.46(\mathrm{~d}, J=8.4 \mathrm{~Hz}, 2 \mathrm{H}) ;{ }^{13} \mathbf{C}$ NMR (100 MHz, CDCl $\left.\mathbf{3}\right): \delta 152.6,150.2$, $138.2,136.7,134.8,130.8,129.1,128.4,128.0,127.8,127.4,127.1,116.6$.

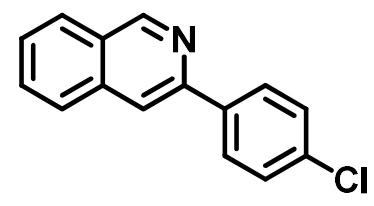


3-(3,4-Dichlorophenyl)isoquinoline (9c): Compound 9c, was prepared following the GP-2 using phthalaldehyde (5a, $1 \mathrm{mmol})$ and $N$-(3,4-dichlorobenzylidene)-4-methylbenzenesulfonamide (7c, 1.2 mmol) as starting material and afforded the title compound 9c (using 10\% ethyl acetate-petroleum ether $(\mathrm{v} / \mathrm{v})$ as an eluent for purification) as thick liquid, Yield: $70 \%(192.0 \mathrm{mg})$; mp $129-130{ }^{\circ} \mathrm{C}$ (lit. ${ }^{51} 132-134$ $\left.{ }^{\circ} \mathrm{C}\right) ;{ }^{1} \mathbf{H}$ NMR (400 MHz, CDCl 3$): \delta 9.30(\mathrm{~s}, 1 \mathrm{H}), 8.24(\mathrm{~s}, 1 \mathrm{H}), 8.01-7.93(\mathrm{~m}, 2 \mathrm{H}), 7.85(\mathrm{t}, J=8 \mathrm{~Hz}, 1 \mathrm{H})$, $7.71(\mathrm{t}, J=8 \mathrm{~Hz}, 1 \mathrm{H}), 7.61(\mathrm{t}, J=4 \mathrm{~Hz}, 1 \mathrm{H}), 7.53(\mathrm{~d}, J=4 \mathrm{~Hz}, 1 \mathrm{H}), 7.38(\mathrm{~d}, J=4 \mathrm{~Hz}, 1 \mathrm{H}) ;{ }^{13} \mathbf{C}$ NMR (100 $\left.\mathbf{M H z}, \mathbf{C D C l}_{3}\right): \delta 152.5,148.4,139.3,136.4,132.9,132.5,130.6,130.0,129.3,128.7,128.0,127.6$, 126.9, 125.9, 116.7; FT-IR $\left(\mathrm{KBr}, \mathrm{cm}^{-1}\right): 1565,1480,1420,1370,1322,1165,1115,1005,890,785,735$; HRMS (ESI-TOF) $\mathrm{m} / \mathrm{z}$ Calcd for $\mathrm{C}_{15} \mathrm{H}_{10} \mathrm{Cl}_{2} \mathrm{~N}[\mathrm{M}+\mathrm{H}]^{+}:$: 274.0190, found 274.0195 (one of the major peaks).

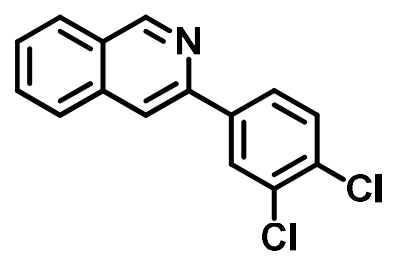

3-(4-Bromophenyl)isoquinoline (9d): ${ }^{3}$ Compound 9d, was prepared following the GP-2 using phthalaldehyde (5a, $1 \mathrm{mmol}$ ) and $\mathrm{N}$-(4-bromobenzylidene)-4-methylbenzenesulfonamide (7d, $1.2 \mathrm{mmol})$ as starting material and afforded the title compound 9d (using 7\% ethyl acetate-petroleum ether (v/v) as an eluent for purification) as brownish solid, Yield: $75 \%(213.0 \mathrm{mg}) ; \mathrm{mp} 150{ }^{\circ} \mathrm{C}$ (lit. $\left.{ }^{3} 145-147{ }^{\circ} \mathrm{C}\right) ;{ }^{1} \mathbf{H}$ NMR (400 MHz, $\left.\mathbf{C D C l}_{3}\right): \delta 9.32(\mathrm{~s}, 1 \mathrm{H}), 8.08-7.98(\mathrm{~m}, 4 \mathrm{H}), 7.92-7.86(\mathrm{~m}, 1 \mathrm{H}), 7.75-7.58(\mathrm{~m}, 4 \mathrm{H}) ;{ }^{13} \mathbf{C}$ NMR (100 MHz, $\left.\mathbf{C D C l}_{3}\right): \delta 152.4,150.0,138.4,136.5,131.9,130.7,128.5,127.8,127.6,127.3,126.9$, $122.9,116.4$.

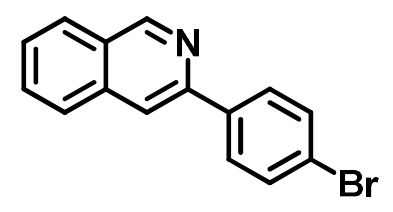

3-(4-Fluorophenyl)isoquinoline (9e): ${ }^{3}$ Compound 9e, was prepared following the GP-2 using phthalaldehyde (5a, $1 \mathrm{mmol})$ and $\mathrm{N}$-(4-fluorobenzylidene)-4-methylbenzenesulfonamide (7e, $1.2 \mathrm{mmol})$ as starting material and afforded the title compound 9e (using 7\% ethyl acetate-petroleum ether (v/v) as an eluent for purification) as pale yellow solid, Yield: $70 \%(156.0 \mathrm{mg}) ; \mathrm{mp} 112{ }^{\circ} \mathrm{C}\left(\right.$ lit. $\left.{ }^{3} 107-109{ }^{\circ} \mathrm{C}\right) ;{ }^{1} \mathbf{H}$ NMR (300 MHz, CDCl 3$): \delta 9.31(\mathrm{~s}, 1 \mathrm{H}), 8.11-8.06(\mathrm{~m}, 2 \mathrm{H}), 7.98(\mathrm{~d}, J=10.5 \mathrm{~Hz}, 2 \mathrm{H}), 7.85(\mathrm{~d}, J=8.1$ $\mathrm{Hz}, 1 \mathrm{H}), 7.72-7.66(\mathrm{~m}, 1 \mathrm{H}), 7.60-7.55(\mathrm{~m}, 1 \mathrm{H}), 7.24-7.14(\mathrm{~m}, 2 \mathrm{H}) ;{ }^{13} \mathbf{C} \mathbf{~ N M R}\left(\mathbf{7 5} \mathbf{~ M H z}, \mathbf{C D C l}_{3}\right): \delta 164.8$ $\left(\mathrm{C}-\mathrm{F},{ }^{1} \mathrm{~J}_{\mathrm{C}-\mathrm{F}}=246 \mathrm{~Hz}\right), 161.6\left(\mathrm{C}-\mathrm{F},{ }^{1} \mathrm{~J}_{\mathrm{C}-\mathrm{F}}=246 \mathrm{~Hz}\right), 152.4,150.3,136.6,135.7\left(\mathrm{C}-\mathrm{F},{ }^{4} \mathrm{~J}_{\mathrm{C}-\mathrm{F}}=3 \mathrm{~Hz}\right), 135.67$ $\left(\mathrm{C}-\mathrm{F},{ }^{4} \mathrm{~J}_{\mathrm{C}-\mathrm{F}}=3 \mathrm{~Hz}\right), 130.8,130.5,128.7,128.6,127.6\left(\mathrm{C}-\mathrm{F},{ }^{3} \mathrm{~J}_{\mathrm{C}-\mathrm{F}}=7.5 \mathrm{~Hz}\right), 127.5\left(\mathrm{C}-\mathrm{F},{ }^{3} \mathrm{~J}_{\mathrm{C}-\mathrm{F}}=7.5 \mathrm{~Hz}\right)$, 127.0, 126.8, 116.1, 115.7 (C-F, $\left.{ }^{2} \mathrm{~J}_{\mathrm{C}-\mathrm{F}}=21.7 \mathrm{~Hz}\right), 115.4\left(\mathrm{C}-\mathrm{F},{ }^{2} \mathrm{~J}_{\mathrm{C}-\mathrm{F}}=21.7 \mathrm{~Hz}\right)$.

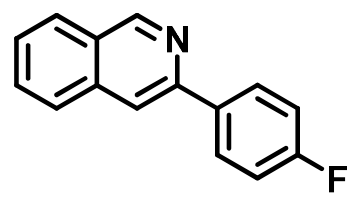

3-(3-Fluorophenyl)isoquinoline (9f): ${ }^{4}$ Compound 9f, was prepared following the GP-2 using phthalaldehyde (5a, $1 \mathrm{mmol}$ ) and $\mathrm{N}$-(3-fluorobenzylidene)-4-methylbenzenesulfonamide (7f, $1.2 \mathrm{mmol}$ ) as starting material and afforded the title compound 9 f (using $7 \%$ ethyl acetate-petroleum ether (v/v) as an 
eluent for purification) as white solid, Yield: 73\% (163.0 mg); mp $98{ }^{\circ} \mathrm{C}$ (lit. ${ }^{4}$ 95-97 $\left.{ }^{\circ} \mathrm{C}\right) ;{ }^{1} \mathbf{H}$ NMR (400 MHz, $\left.\mathbf{C D C l}_{3}\right): \delta 9.47(\mathrm{~s}, 1 \mathrm{H}), 8.05(\mathrm{~d}, J=8 \mathrm{~Hz}, 1 \mathrm{H}), 7.91-7.43(\mathrm{~m}, 3 \mathrm{H}), 7.41(\mathrm{t}, J=4 \mathrm{~Hz}, 1 \mathrm{H}), 7.38-7.27$ $(\mathrm{m}, 2 \mathrm{H}), 7.14(\mathrm{t}, J=4 \mathrm{~Hz}, 1 \mathrm{H}), 7.10(\mathrm{t}, J=8 \mathrm{~Hz}, 1 \mathrm{H}) ;{ }^{13} \mathbf{C}$ NMR $\left(\mathbf{7 5} \mathbf{M H z}, \mathbf{C D C l}_{3}\right): \delta 165.0\left(\mathrm{C}-\mathrm{F},{ }^{1} J_{\mathrm{C}-\mathrm{F}}=\right.$ $243.7 \mathrm{~Hz}), 161.8\left(\mathrm{C}-\mathrm{F},{ }^{1} J_{\mathrm{C}-\mathrm{F}}=243.7 \mathrm{~Hz}\right), 152.5,149.9\left(\mathrm{C}-\mathrm{F},{ }^{4} J_{\mathrm{C}-\mathrm{F}}=3 \mathrm{~Hz}\right), 149.8\left(\mathrm{C}-\mathrm{F},{ }^{4} J_{\mathrm{C}-\mathrm{F}}=3 \mathrm{~Hz}\right)$, $142.0\left(\mathrm{C}-\mathrm{F},{ }^{3} J_{\mathrm{C}-\mathrm{F}}=7.5 \mathrm{~Hz}\right), 141.9\left(\mathrm{C}-\mathrm{F},{ }^{3} J_{\mathrm{C}-\mathrm{F}}=7.5 \mathrm{~Hz}\right), 136.5,130.7,130.3\left(\mathrm{C}-\mathrm{F},{ }^{3} J_{\mathrm{C}-\mathrm{F}}=8.2 \mathrm{~Hz}\right), 130.2$ $\left(\mathrm{C}-\mathrm{F},{ }^{3} J_{\mathrm{C}-\mathrm{F}}=8.2 \mathrm{~Hz}\right), 128.0,127.6,127.4,127.0,122.5,116.8,115.5\left(\mathrm{C}-\mathrm{F},{ }^{2} J_{\mathrm{C}-\mathrm{F}}=21 \mathrm{~Hz}\right), 115.2(\mathrm{C}-\mathrm{F}$, $\left.{ }^{2} J_{\mathrm{C}-\mathrm{F}}=21 \mathrm{~Hz}\right), 114.1\left(\mathrm{C}-\mathrm{F},{ }^{2} J_{\mathrm{C}-\mathrm{F}}=22.5 \mathrm{~Hz}\right), 113.8\left(\mathrm{C}-\mathrm{F},{ }^{2} J_{\mathrm{C}-\mathrm{F}}=22.5 \mathrm{~Hz}\right)$.

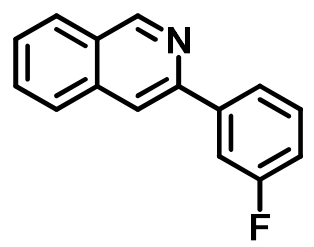

4-(Isoquinolin-3-yl)benzonitrile (9g): ${ }^{3}$ Compound $9 \mathrm{~g}$, was prepared following the GP-2 using phthalaldehyde (5a, $1 \mathrm{mmol})$ and $N$-(4-cyanobenzylidene)-4-methylbenzenesulfonamide (7g, $1.2 \mathrm{mmol})$ as starting material and afforded the title compound $9 \mathrm{~g}$ (using $8 \%$ ethyl acetate-petroleum ether (v/v) as an eluent for purification) as brownish solid, Yield: $65 \%(150.0 \mathrm{mg}) ; \mathrm{mp} 172{ }^{\circ} \mathrm{C}$ (lit. $\left.{ }^{3} 166-168{ }^{\circ} \mathrm{C}\right) ;{ }^{1} \mathbf{H}$ NMR (400 MHz, CDCl $\left.\mathbf{~}_{3}\right): \delta 9.34(\mathrm{~s}, 1 \mathrm{H}), 8.21(\mathrm{~d}, J=8 \mathrm{~Hz}, 2 \mathrm{H}), 8.10(\mathrm{~s}, 1 \mathrm{H}), 8.00(\mathrm{~d}, J=8 \mathrm{~Hz}, 1 \mathrm{H})$, $7.90(\mathrm{t}, J=8 \mathrm{~Hz}, 1 \mathrm{H}), 7.76-7.72(\mathrm{~m}, 2 \mathrm{H}), 7.67-7.64(\mathrm{~m}, 1 \mathrm{H}) ;{ }^{13} \mathbf{C}$ NMR (100 MHz, CDCl $): \delta 152.6$, $148.7,146.4,143.5,136.3,132.5,129.0,131.0,128.0,127.6,127.4,127.0,118.8,117.7,111.8$.

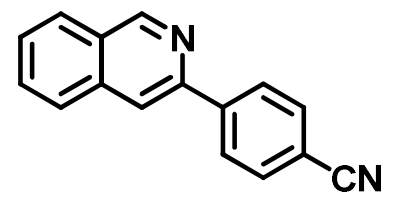

3-(2,5-Dimethylphenyl)isoquinoline (9h): Compound 9h, was prepared following the GP-2 using phthalaldehyde (5a, $1 \mathrm{mmol})$ and $N$-(2,5-dimethylbenzylidene)-4-methylbenzenesulfonamide (7h, 1.2 mmol) as starting material and afforded the title compound $\mathbf{9 h}$ (using 6\% ethyl acetate-petroleum ether (v/v) as an eluent for purification) as viscous liquid, Yield: $85 \%(198.0 \mathrm{mg}) ;{ }^{1} \mathbf{H}$ NMR (400 MHz, $\left.\mathbf{C D C l}_{3}\right): \delta 9.13(\mathrm{~s}, 1 \mathrm{H}), 8.02(\mathrm{~d}, J=8 \mathrm{~Hz}, 2 \mathrm{H}), 7.36(\mathrm{~d}, J=8.4 \mathrm{~Hz}, 2 \mathrm{H}), 7.26(\mathrm{~d}, J=13.2 \mathrm{~Hz}, 2 \mathrm{H}), 7.15(\mathrm{~d}$, $J=7.6 \mathrm{~Hz}, 1 \mathrm{H}), 7.07$ (d, $J=7.6 \mathrm{~Hz}, 1 \mathrm{H}), 2.49(\mathrm{~s}, 3 \mathrm{H}), 2.29(\mathrm{~d}, J=8.4 \mathrm{~Hz}, 3 \mathrm{H}) ;{ }^{13} \mathbf{C} \mathbf{~ N M R}(\mathbf{7 5} \mathbf{M H z}$, $\left.\mathbf{C D C l}_{3}\right): \delta 153.0,152.0,137.4,135.8,134.3,133.8,132.1,131.5,131.3,130.4,128.5,127.6,127.2,126.3$, 116.9, 21.0, 20.9; FT-IR (KBr, cm $\left.{ }^{-1}\right): 2924,1577,1520,1450,1280,850,790,750$; HRMS (ESI-TOF) $\mathrm{m} / \mathrm{z}$ Calcd for $\mathrm{C}_{17} \mathrm{H}_{16} \mathrm{~N}[\mathrm{M}+\mathrm{H}]^{+}: 234.1283$, found 234.1286 .

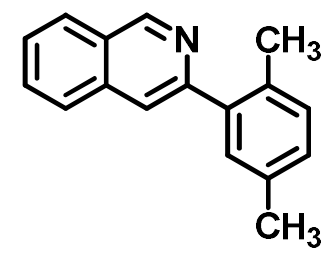

3-(2-Methoxyphenyl)isoquinoline (9i): ${ }^{4}$ Compound 9i, was prepared following the GP-2 using phthalaldehyde (5a, $1 \mathrm{mmol}$ ) and $N$-(2-methoxybenzylidene)-4-methylbenzenesulfonamide (7i, 1.2 mmol) as starting material and afforded the title compound 9i (using 7\% ethyl acetate-petroleum ether (v/v) as an eluent for purification) as viscous liquid, Yield: 83\% (195.0 mg); ${ }^{\mathbf{1}} \mathbf{H}$ NMR (400 MHz, 
$\left.\mathbf{C D C l}_{3}\right): \delta 9.35(\mathrm{~s}, 1 \mathrm{H}), 8.20(\mathrm{~s}, 1 \mathrm{H}), 7.99(\mathrm{~d}, J=8 \mathrm{~Hz}, 1 \mathrm{H}), 7.92-7.85(\mathrm{~m}, 2 \mathrm{H}), 7.68(\mathrm{t}, J=8 \mathrm{~Hz}, 1 \mathrm{H})$, $7.59(\mathrm{~d}, J=8 \mathrm{~Hz}, 1 \mathrm{H}), 7.38(\mathrm{t}, J=8.4 \mathrm{~Hz}, 1 \mathrm{H}), 7.13(\mathrm{~d}, J=7.2 \mathrm{~Hz}, 1 \mathrm{H}), 7.05(\mathrm{~d}, J=8.4 \mathrm{~Hz}, 1 \mathrm{H}), 3.90(\mathrm{~s}$, 3H); ${ }^{13}$ C NMR (100 MHz, $\left.\mathbf{C D C l}_{3}\right): \delta 157.3,152.0,149.4,136.4,134.0,131.6,130.4,130.1,129.6,129.5$, $129.3,127.6,127.5,127.2,127.1,123.4,121.3,121.2,111.7,55.9$.

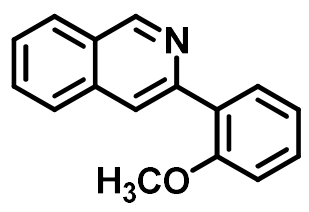

3-(4-Methoxyphenyl)isoquinoline (9j): Compound 9j, was prepared following the GP-2 using phthalaldehyde (5a, $1 \mathrm{mmol})$ and $N$-(4-methoxybenzylidene)-4-methylbenzenesulfonamide (7j, 1.2 mmol) as starting material and afforded the title compound $\mathbf{9 j}$ (using $10 \%$ ethyl acetate-petroleum ether $(\mathrm{v} / \mathrm{v})$ as an eluent for purification) as yellow liquid, Yield: $85 \%(200.0 \mathrm{mg}) ;{ }^{1} \mathbf{H}$ NMR (400 $\mathbf{~ M H z}$, $\left.\mathbf{C D C l}_{3}\right): \delta 9.35(\mathrm{~s}, 1 \mathrm{H}), 8.13(\mathrm{t}, J=1.2 \mathrm{~Hz}, 1 \mathrm{H}), 8.07(\mathrm{~s}, 1 \mathrm{H}), 8.00(\mathrm{~d}, J=8 \mathrm{~Hz}, 1 \mathrm{H}), 7.88(\mathrm{~d}, J=8 \mathrm{~Hz}$, $1 \mathrm{H}), 7.70(\mathrm{~s}, 1 \mathrm{H}), 7.60(\mathrm{~d}, J=8 \mathrm{~Hz}, 1 \mathrm{H}), 7.51(\mathrm{t}, J=7.6 \mathrm{~Hz}, 2 \mathrm{H}), 7.43(\mathrm{~d}, J=7.2 \mathrm{~Hz}, 1 \mathrm{H}), 3.90(\mathrm{~s}, 3 \mathrm{H})$; ${ }^{13} \mathbf{C}$ NMR (100 MHz, $\left.\mathbf{C D C l}_{3}\right): \delta$ 152.5. 151.5, 139.7, 136.8, 130.7, 128.9, 128.7, 127.9, 127.7, 127.2, 127.1, 116.7, 55.8; HRMS (ESI-TOF) m/z Calcd for $\mathrm{C}_{16} \mathrm{H}_{14} \mathrm{NO}[\mathrm{M}+\mathrm{H}]^{+}:$236.1075, found 236.1079.

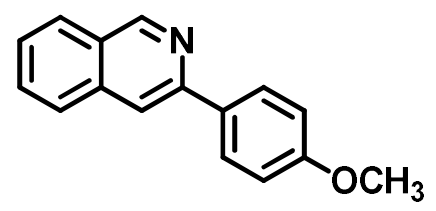

3-p-Tolylisoquinoline (9k): ${ }^{3}$ Compound 9k, was prepared following the GP-2 using phthalaldehyde (5a, $1 \mathrm{mmol})$ and 4-Methyl- $N$-(4-methylbenzylidene)benzenesulfonamide $(7 \mathbf{k}, 1.2 \mathrm{mmol})$ as starting material and afforded the title compound $9 \mathbf{k}$ (using $5 \%$ ethyl acetate-petroleum ether $(\mathrm{v} / \mathrm{v})$ as an eluent for purification) as white solid, Yield: $83 \%(182.0 \mathrm{mg})$; mp $76{ }^{\circ} \mathrm{C}$ (lit. $\left.{ }^{3} 71-73{ }^{\circ} \mathrm{C}\right) ;{ }^{1} \mathbf{H}$ NMR (400 MHz, $\left.\mathbf{C D C l}_{3}\right): \delta 9.33(\mathrm{~s}, 1 \mathrm{H}), 8.04-7.97(\mathrm{~m}, 4 \mathrm{H}), 7.86(\mathrm{~d}, J=8 \mathrm{~Hz}, 1 \mathrm{H}), 7.69(\mathrm{t}, J=6.8 \mathrm{~Hz}, 1 \mathrm{H}), 7.57(\mathrm{t}, J=8$ $\mathrm{Hz}, 1 \mathrm{H}), 2.43(\mathrm{~s}, 3 \mathrm{H}), 7.32(\mathrm{~d}, J=8 \mathrm{~Hz}, 2 \mathrm{H}) ;{ }^{13} \mathbf{C}$ NMR (100 MHz, $\left.\mathbf{C D C l}_{3}\right): \delta 152.4,151.5,138.6$, $136.92,136.87,130.6,129.7,127.8,127.7,127.03,127.0,116.2,21.4$.

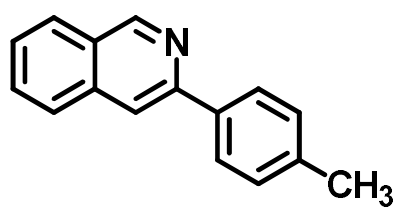

3-(Pyren-1-yl)isoquinoline (9l): Compound 9l, was prepared following the GP-2 using phthalaldehyde (5a, $1 \mathrm{mmol}$ ) and 4-Methyl- $N$-(pyren-1-ylmethylene)benzenesulfonamide (7l, $1.2 \mathrm{mmol}$ ) as starting material and afforded the title compound 91 (using 12\% ethyl acetate-petroleum ether $(\mathrm{v} / \mathrm{v})$ as an eluent for purification) as pale yellow viscous liquid, Yield: $80 \%(263.0 \mathrm{mg}) ;{ }^{1} \mathbf{H}$ NMR (400 $\left.\mathbf{~ M H z}, \mathbf{C D C l}_{3}\right): \delta$ $9.38(\mathrm{~s}, 1 \mathrm{H}), 8.41(\mathrm{~d}, J=8 \mathrm{~Hz}, 2 \mathrm{H}), 8.30-8.20(\mathrm{~m}, 7 \mathrm{H}), 8.14-8.05(\mathrm{~m}, 5 \mathrm{H}) ;{ }^{13} \mathbf{C}$ NMR (100 MHz, $\left.\mathbf{C D C l}_{3}\right): \delta 152.6,151.5,134.1,132.9,130.8,130.5,130.4,129.5,127.0,126.92,126.89,126.79,124.3$, 123.9, 123.4, 118.8; FT-IR (neat, $\mathrm{cm}^{-1}$ ): 1586, 1565, 1545, 1446, 1390, 1344, 840, 815, 795, 755, 720; HRMS (ESI-TOF) $\mathrm{m} / \mathrm{z}$ Calcd for $\mathrm{C}_{25} \mathrm{H}_{16} \mathrm{~N}[\mathrm{M}+\mathrm{H}]+:$ 330.1283, found 330.1281. 


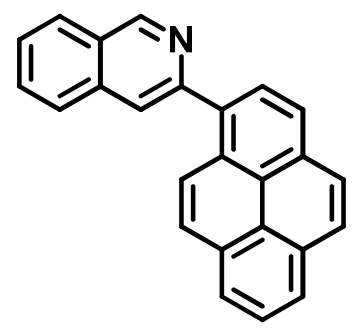

3-(Thiophen-2-yl)isoquinoline (9m): ${ }^{3}$ Compound $9 \mathrm{~m}$, was prepared following the GP-2 using phthalaldehyde (5a, $1 \mathrm{mmol}$ ) and $N$-(thiophen-2-ylmethylene)-4-methylbenzenesulfonamide (7m, 1.2 $\mathrm{mmol}$ ) as starting material and afforded the title compound $\mathbf{9 m}$ (using 15\% ethyl acetate-petroleum ether $(\mathrm{v} / \mathrm{v})$ as an eluent for purification) as brownish solid, Yield: $83 \%(175.0 \mathrm{mg}) ; \mathrm{mp} 110{ }^{\circ} \mathrm{C}$ (lit. ${ }^{3} 111-113$ $\left.{ }^{\circ} \mathrm{C}\right) ;{ }^{1} \mathbf{H}$ NMR (300 MHz, CDCl 3$): \delta 9.27(\mathrm{~s}, 1 \mathrm{H}), 7.98(\mathrm{~d}, J=10.5 \mathrm{~Hz}, 2 \mathrm{H}), 7.85(\mathrm{~d}, J=8.4 \mathrm{~Hz}, 1 \mathrm{H})$, $7.75-7.68(\mathrm{~m}, 2 \mathrm{H}), 7.58(\mathrm{t}, J=7.5 \mathrm{~Hz}, 1 \mathrm{H}), 7.43(\mathrm{~d}, J=5.1 \mathrm{~Hz}, 1 \mathrm{H}), 7.18(\mathrm{t}, J=3.9 \mathrm{~Hz}, 1 \mathrm{H}) ;{ }^{13} \mathbf{C} \mathbf{N M R}$ (75 MHz, $\left.\mathbf{C D C l}_{3}\right): \delta 152.3,146.3,144.6,136.6,131.1,128.3,127.9,127.7,127.0,126.98,126.7,124.2$, 114.7 .

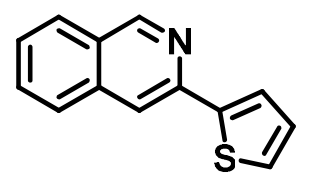

3-(Furan-2-yl)isoquinoline (9n): ${ }^{3}$ Compound 9n, was prepared following the GP-2 using phthalaldehyde (5a, $1 \mathrm{mmol}$ ) and $N$-(furan-2-ylmethylene)-4-methylbenzenesulfonamide $(\mathbf{7 n}, 1.2 \mathrm{mmol})$ as starting material and afforded the title compound 9n (using 15\% ethyl acetate-petroleum ether $(\mathrm{v} / \mathrm{v})$ as an eluent for purification) as yellow solid, Yield: $78 \%(152.0 \mathrm{mg})$; $\mathrm{mp} 71-73{ }^{\circ} \mathrm{C}$ (lit. $\left.{ }^{3} 66-68{ }^{\circ} \mathrm{C}\right) ;{ }^{1} \mathbf{H}$ NMR (400 MHz, $\left.\mathbf{C D C l}_{3}\right): \delta 9.23(\mathrm{~s}, 1 \mathrm{H}), 8.02(\mathrm{~s}, 1 \mathrm{H}), 7.94(\mathrm{~d}, J=8 \mathrm{~Hz}, 1 \mathrm{H}), 7.84(\mathrm{~d}, J=8 \mathrm{~Hz}, 1 \mathrm{H}), 7.68(\mathrm{t}, J=8$ $\mathrm{Hz}, 1 \mathrm{H}), 7.55$ (t, $J=8 \mathrm{~Hz}, 2 \mathrm{H}), 7.12(\mathrm{~d}, J=4 \mathrm{~Hz}, 1 \mathrm{H}), 6.56-6.55(\mathrm{~m}, 1 \mathrm{H}) ;{ }^{13} \mathbf{C} \mathbf{N M R}\left(\mathbf{1 0 0} \mathbf{M H z}, \mathbf{C D C l}_{3}\right)$ : $\delta 154.0,152.6,143.3,143.2,136.6,131.0,128.0,127.8,127.1,127.0,114.4,112.3,108.6$.

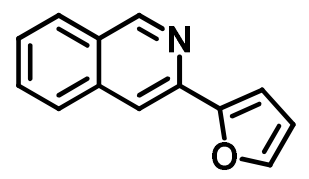

3-(Pyridin-3-yl)isoquinoline (90): ${ }^{3}$ Compound 9o, was prepared following the GP-2 using phthalaldehyde (5a, $1 \mathrm{mmol}$ ) and 4-Methyl- $N$-(pyridin-3-ylmethylene)benzenesulfonamide (7o, 1.2 mmol) as starting material and afforded the title compound 90 (using 15\% ethyl acetate-petroleum ether (v/v) as an eluent for purification) as yellow solid, Yield: $80 \%(165.0 \mathrm{mg}) ; \mathrm{mp} 76{ }^{\circ} \mathrm{C}\left(\mathrm{lit} .{ }^{3} 78-80{ }^{\circ} \mathrm{C}\right) ;{ }^{1} \mathbf{H}$ NMR (400 MHz, CDCl $\left.\mathbf{M}_{3}\right): \delta 9.34(\mathrm{~s}, 1 \mathrm{H}), 9.29(\mathrm{~s}, 1 \mathrm{H}), 8.62(\mathrm{~d}, J=3.2 \mathrm{~Hz}, 1 \mathrm{H}), 8.47(\mathrm{~d}, J=6.4 \mathrm{~Hz}, 1 \mathrm{H})$, $8.10(\mathrm{~s}, 1 \mathrm{H}), 8.01(\mathrm{~d}, J=6.4 \mathrm{~Hz}, 1 \mathrm{H}), 7.90(\mathrm{~d}, J=6.4 \mathrm{~Hz}, 1 \mathrm{H}), 7.78(\mathrm{t}, J=6.4 \mathrm{~Hz}, 1 \mathrm{H}), 7.73(\mathrm{t}, J=6.4$ $\mathrm{Hz}, 1 \mathrm{H}), 7.67-7.62(\mathrm{~m}, 1 \mathrm{H}), 7.53(\mathrm{t}, J=2.4 \mathrm{~Hz}, 1 \mathrm{H}), 7.46-7.44(\mathrm{~m}, 1 \mathrm{H}), 7.71-7.31(\mathrm{~m}, 1 \mathrm{H}) ;{ }^{13} \mathbf{C} \mathbf{N M R}$ (100 MHz, $\left.\mathbf{C D C l}_{3}\right): \delta 153.0,148.9,148.3,147.9,136.6,135.5,135.0,131.1,129.9,129.8,128.3,128.2$, $127.9,127.8,127.1,126.6,123.9,117.2$.<smiles>c1cncc(-c2cc3ccccc3cn2)c1</smiles> 
3-(Pyridin-4-yl)isoquinoline (9p): ${ }^{3}$ Compound $\mathbf{9 p}$, was prepared following the GP-2 using phthalaldehyde (5a, $1 \mathrm{mmol})$ and 4-Methyl- $N$-(pyridin-4-ylmethylene)benzenesulfonamide (7p, 1.2 mmol) as starting material and afforded the title compound $9 \mathbf{p}$ (using 15\% ethyl acetate-petroleum ether $(\mathrm{v} / \mathrm{v})$ as an eluent for purification) as yellow solid, Yield: $81 \%(167.0 \mathrm{mg})$; mp 130-132 ${ }^{\circ} \mathrm{C}$ (lit. ${ }^{3} 125-127$ $\left.{ }^{\circ} \mathrm{C}\right) ;{ }^{1} \mathbf{H}$ NMR (400 MHz, $\left.\mathbf{C D C l}_{3}\right): \delta 9.37(\mathrm{~s}, 1 \mathrm{H}), 8.74-8.71(\mathrm{~m}, 2 \mathrm{H}), 8.21(\mathrm{~s}, 1 \mathrm{H}), 8.09(\mathrm{~d}, J=8 \mathrm{~Hz}, 1 \mathrm{H})$, $8.04(\mathrm{~d}, J=8 \mathrm{~Hz}, 1 \mathrm{H}), 7.94(\mathrm{~d}, J=8 \mathrm{~Hz}, 1 \mathrm{H}), 7.79-7.75(\mathrm{~m}, 1 \mathrm{H}), 7.68(\mathrm{t}, J=4 \mathrm{~Hz}, 1 \mathrm{H}), 7.61-7.56(\mathrm{~m}$, $1 \mathrm{H}) ;{ }^{13} \mathbf{C}$ NMR (100 MHz, $\left.\mathbf{C D C l}_{3}\right): \delta 153.1,150.4,149.7,136.4,135.3,131.2,130.1,129.5,128.8,128.4$, $127.4,126.7,121.5,119.1,118.1$.

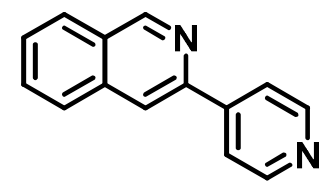

3-(Pyridin-4-yl)isoquinoline (9q): Compound 9q, was prepared following the GP-2 using 4methoxyphthalaldehyde $(\mathbf{5 b}, 1 \mathrm{mmol})$ and $N$-(4-chlorobenzylidene)-4-methylbenzenesulfonamide $(\mathbf{7 q}$, $1.2 \mathrm{mmol}$ ) as starting material and afforded the title compound $\mathbf{9 q}$ (using 12\% ethyl acetate-petroleum ether (v/v) as an eluent for purification) as pale yellow viscous liquid, Yield: $75 \%(202.0 \mathrm{mg}) ;{ }^{1} \mathbf{H}$ NMR $\left(300 \mathbf{M H z}, \mathbf{C D C l}_{3}\right): \delta 9.20(\mathrm{~s}, 1 \mathrm{H}), 8.06(\mathrm{~d}, J=8.7 \mathrm{~Hz}, 2 \mathrm{H}), 7.96(\mathrm{~s}, 1 \mathrm{H}), 7.90(\mathrm{~d}, J=9 \mathrm{~Hz}, 1 \mathrm{H}), 7.48(\mathrm{~d}$, $J=8.4 \mathrm{~Hz}, 2 \mathrm{H}), 7.28-7.23(\mathrm{~m}, 1 \mathrm{H}), 7.14(\mathrm{~d}, J=2.1 \mathrm{~Hz}, 1 \mathrm{H}), 4.00(\mathrm{~s}, 3 \mathrm{H}) ;{ }^{13} \mathbf{C} \mathbf{N M R}\left(75 \mathbf{~ M H z}, \mathbf{C D C l}_{3}\right)$ : $\delta$ 161.3, 151.6, 150.6, 138.6, 138.2, 134.6, 130.9, 129.3, 128.9, 128.3, 123.7, 120.4, 115.9, 104.5, 55.5; FT-IR (neat, $\mathrm{cm}^{-1}$ ): 1580, 1545, 1475, 1425, 1375, 1321, 1310, 1200, 1160, 1110, 1005, 890, 790, 740; HRMS (ESI-TOF) $\mathrm{m} / \mathrm{z}$ Calcd for $\mathrm{C}_{16} \mathrm{H}_{13} \mathrm{ClNO}[\mathrm{M}+\mathrm{H}]+:$ 270.0686, found 270.0690.

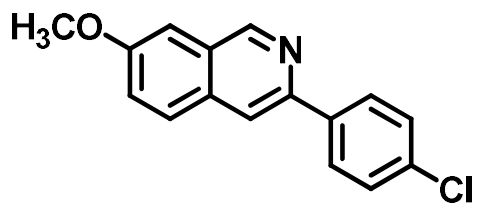


Section IV: ESI-MS kinetics data for detecting the intermediates during the formation of $6 f$

From our ESI-MS reaction kinetics spectrum (Fig. S1, S2, S3, S4) of the on-going reaction among 5a, 1f and 11c, symbolic ESI-MS peaks were detected for 'Breslow intermediate' I, i.e., 11c-5a adduct at e/m 306.1034, intermediate II at e/m 701.2092, intermediate III at e/m 701.2039 and $\mathbf{6 f}$ at e/m 534.0912.

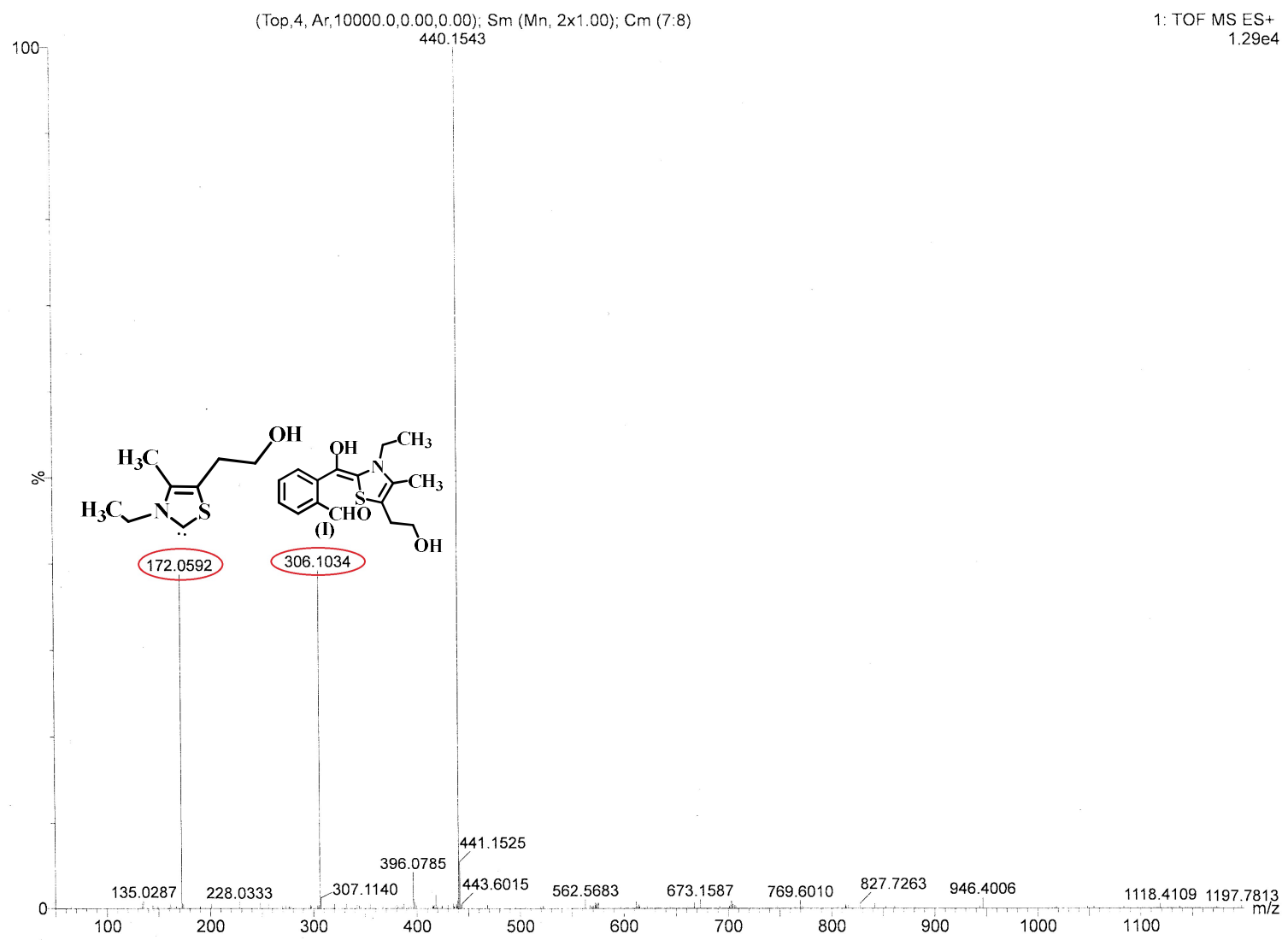

Figure S1. ESI-MS spectrum of the ongoing reaction after 3 min (intermediate I). 


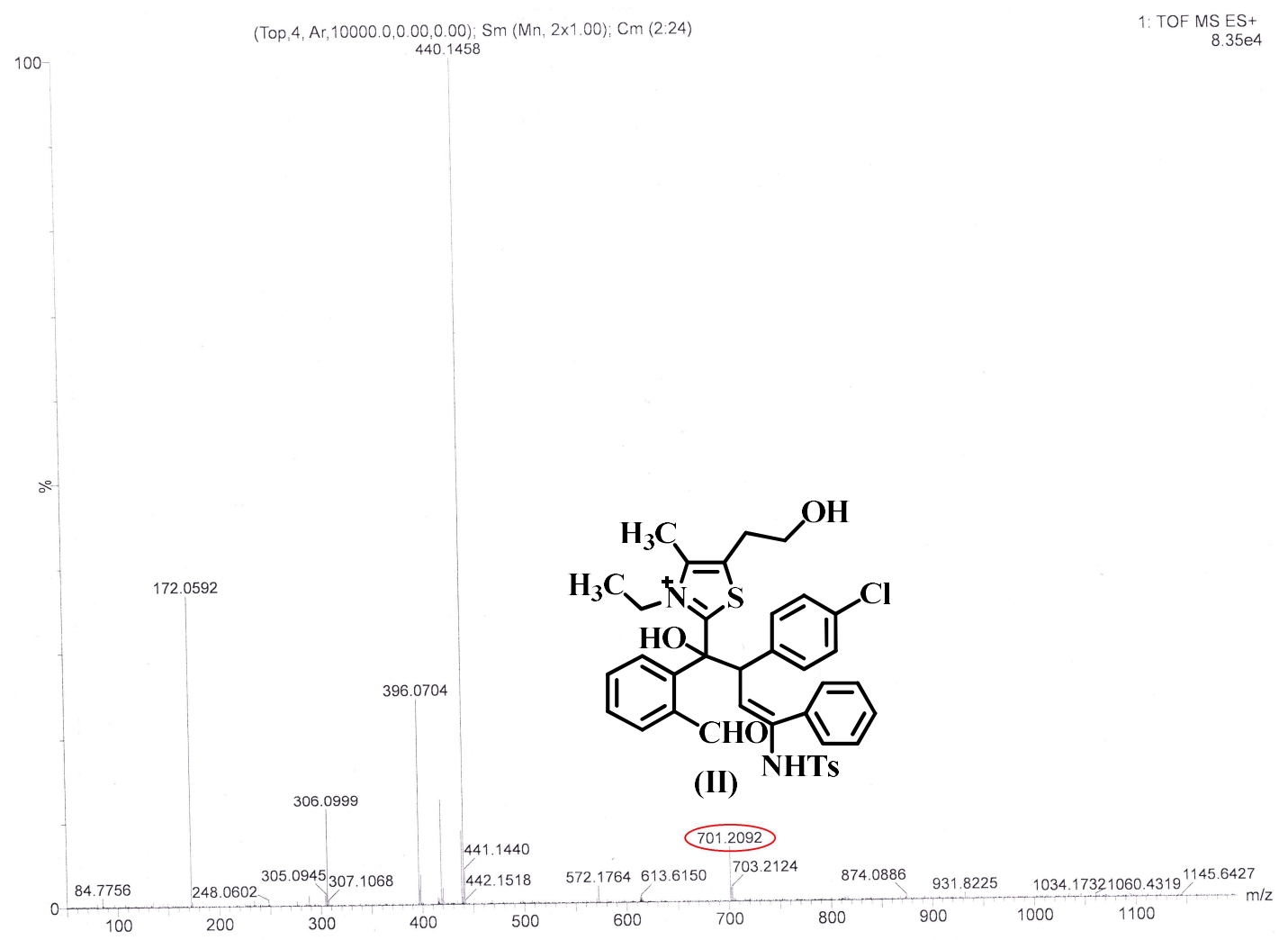

Figure S2. ESI-MS spectrum of the ongoing reaction after 30 min (intermediate II).

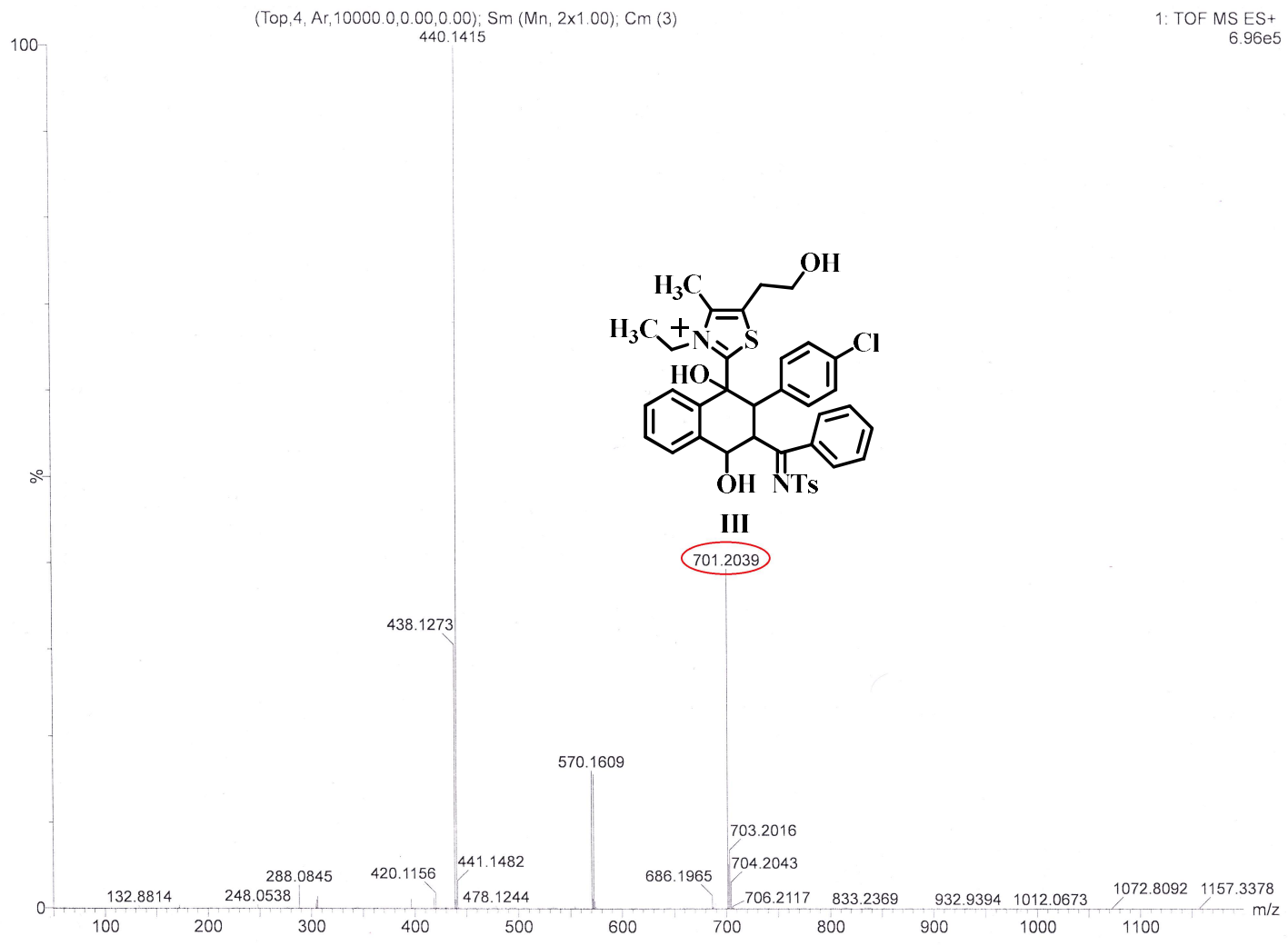

Figure S3. ESI-MS spectrum of the ongoing reaction after 60 min (intermediate III). 


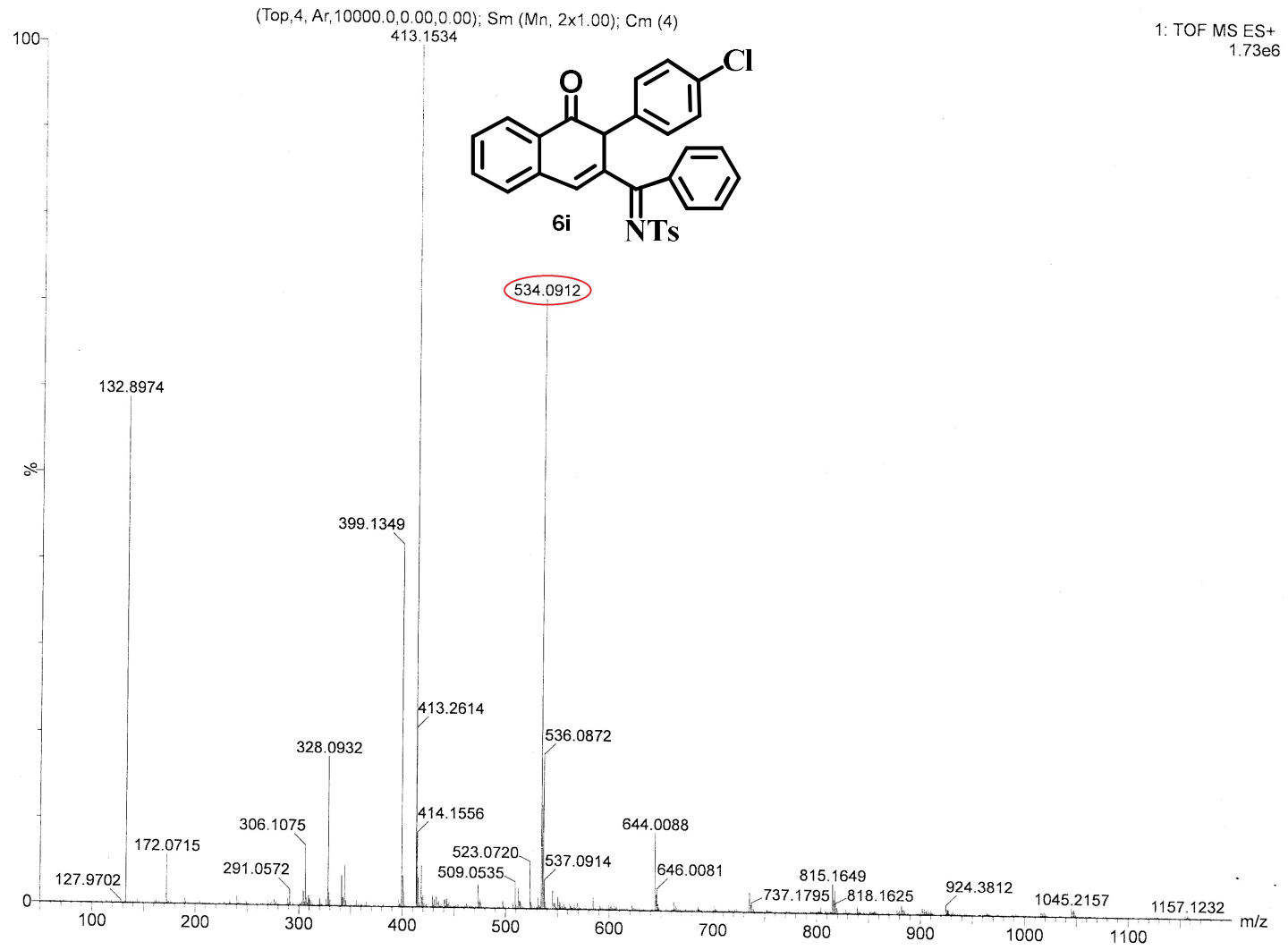

Figure S4. ESI-MS spectrum of the ongoing reaction after 120 min (intermediate 6i) (product formation started). 
Section V: ESI-MS data for detecting the intermediates during the formation of 9b

From our ESI-MS reaction kinetics (Fig. S5, S6, S7. S8, S9) of the ongoing reaction among compound 7 and NHC 11c $(\mathrm{R}=4-\mathrm{Cl})$, characteristic ESI-MS peaks were detected for intermediate $\mathrm{V}$ at e/m 465.1149 , intermediate VI at e/m 599.1497, VIII at e/m 433.0803, 12 at e/m 188.0561 and $\mathbf{9 b}$ at e/m 240.0384.

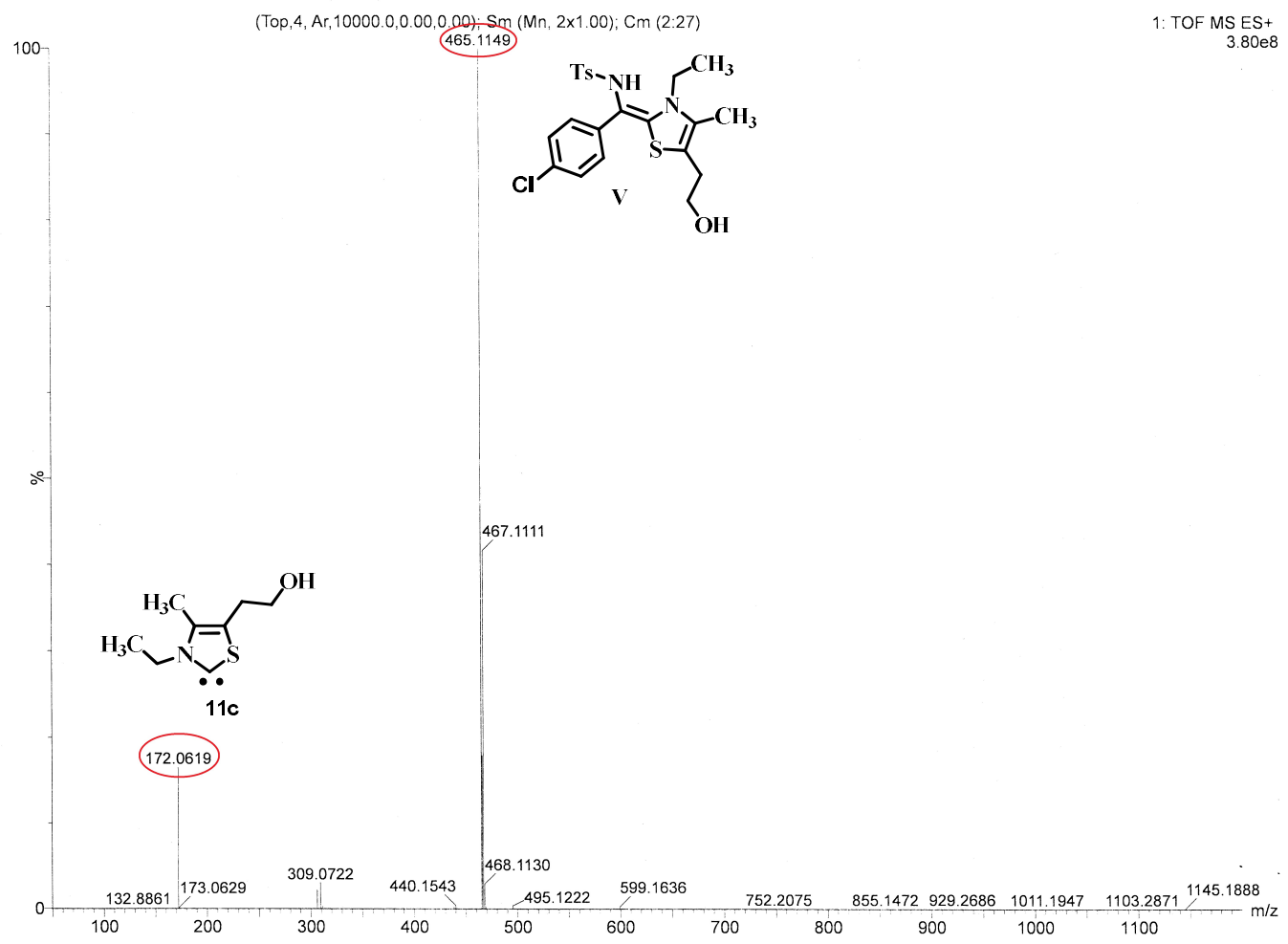

Figure S5. ESI-MS spectrum of the ongoing after 3 min (intermediate V). 


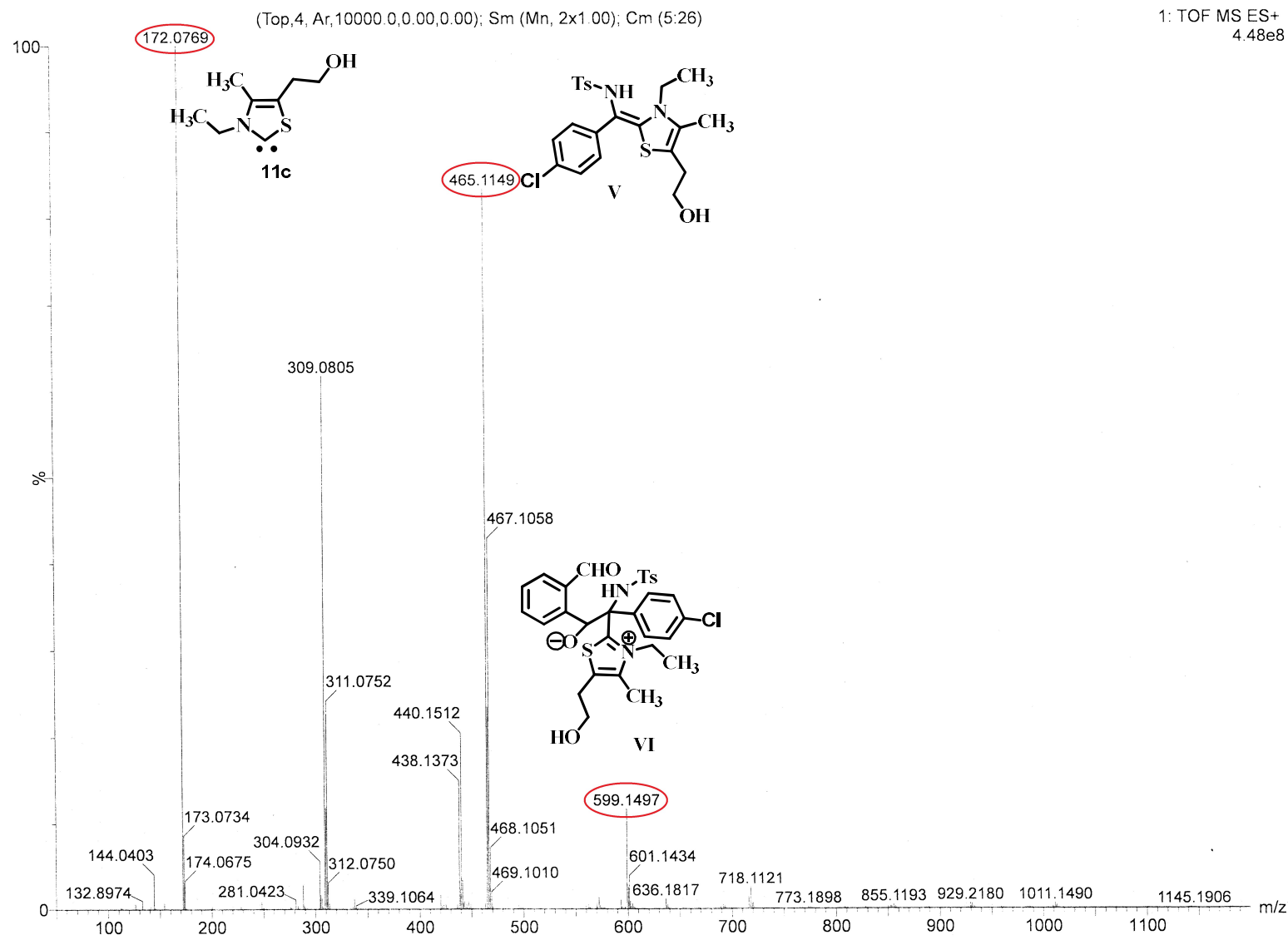

Figure S6. ESI-MS spectrum of the ongoing after $20 \mathrm{~min}$ (intermediate $\mathrm{V}$ and VI).

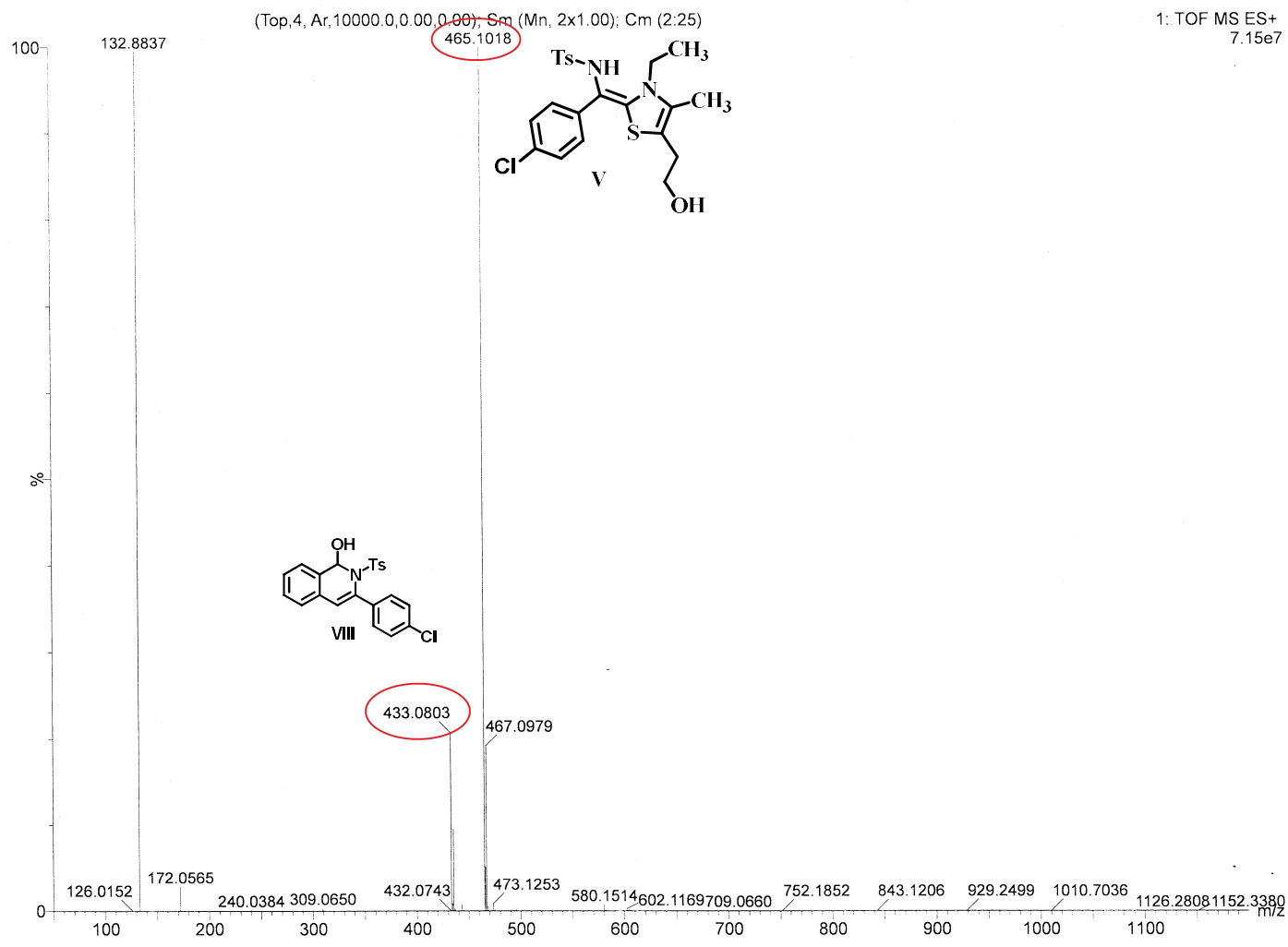

Figure S7. ESI-MS spectrum of the ongoing after $90 \mathrm{~min}$ (intermediate V and VIII). 


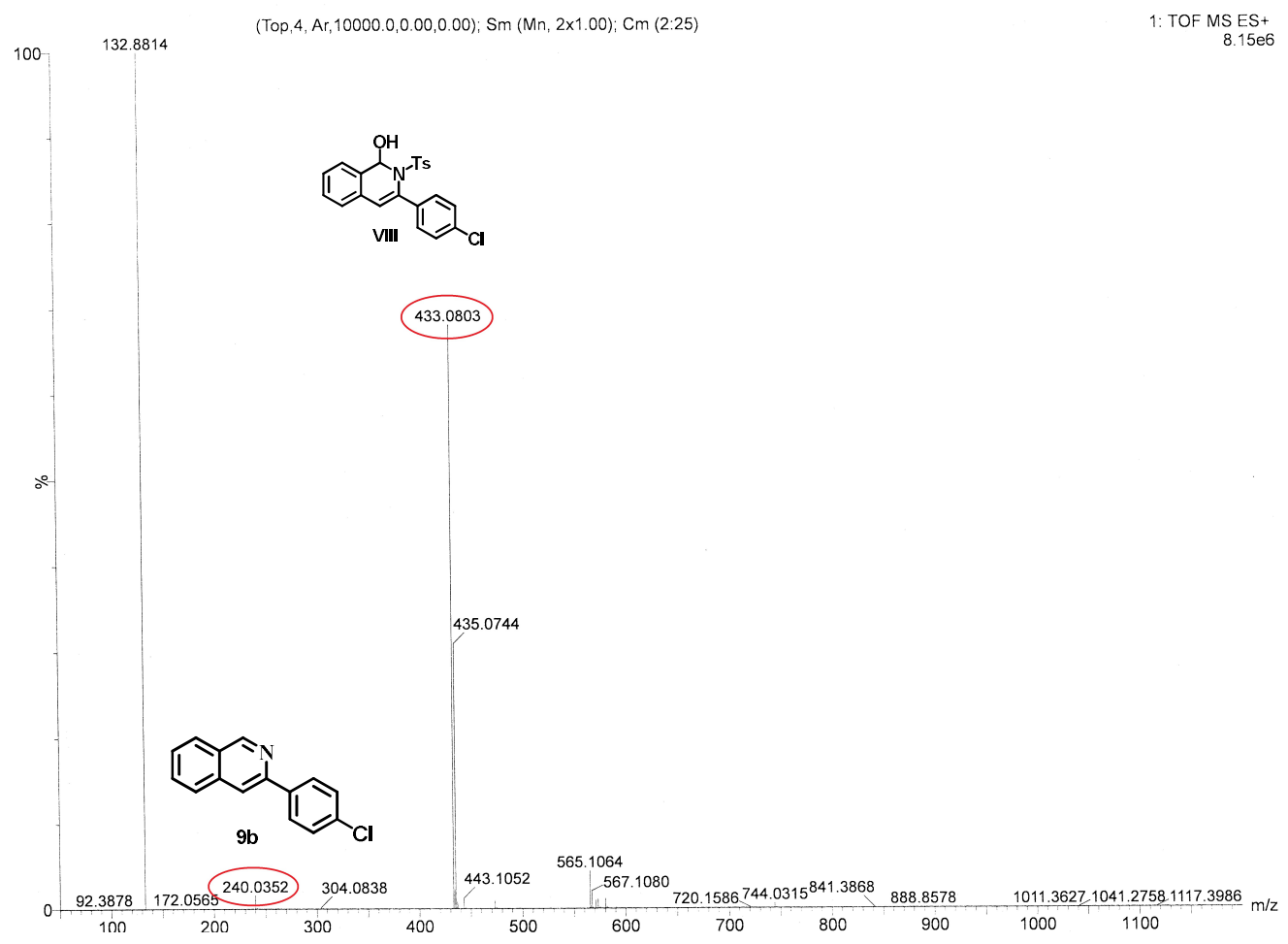

Figure S8. ESI-MS spectrum of the ongoing after $150 \mathrm{~min}$ (intermediate VIII and final compound $\mathbf{9 b}$ ).

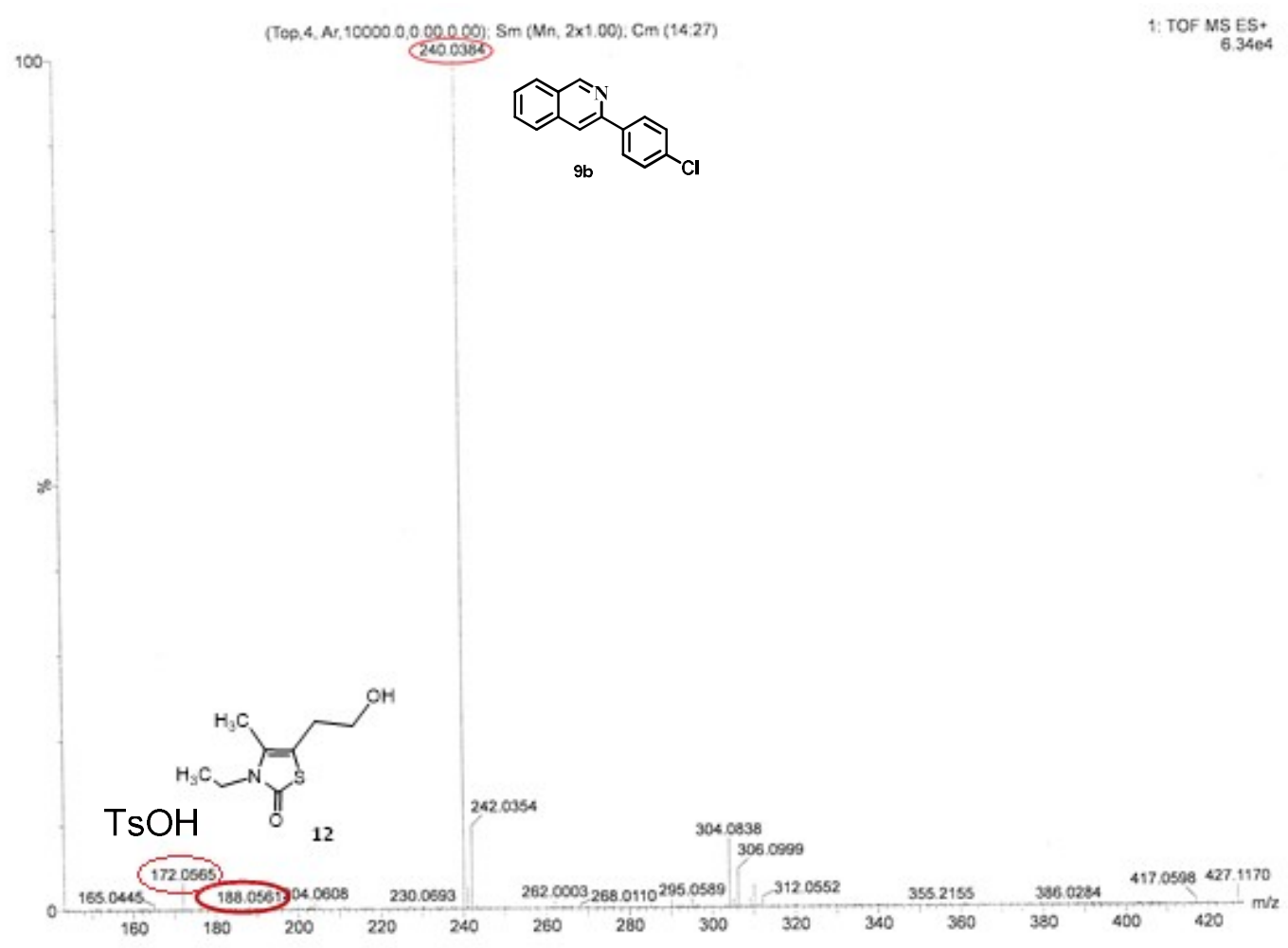

Figure S9. ESI-MS spectrum of the ongoing after $180 \mathrm{~min}$ (12 and final compound $\mathbf{9 b}$ ).

S-22 
Section VI: ${ }^{1} \mathrm{H}$ and ${ }^{13} \mathrm{C}-\mathrm{NMR}$ spectra of substituted naphthalen-1(2H)-one derivatives (6a-t) ${ }^{1} \mathrm{H}$ NMR (400 MHz) and ${ }^{13} \mathrm{C}$ NMR (100 MHz) spectra of compound 6a in $\mathrm{CDCl}_{3}$.

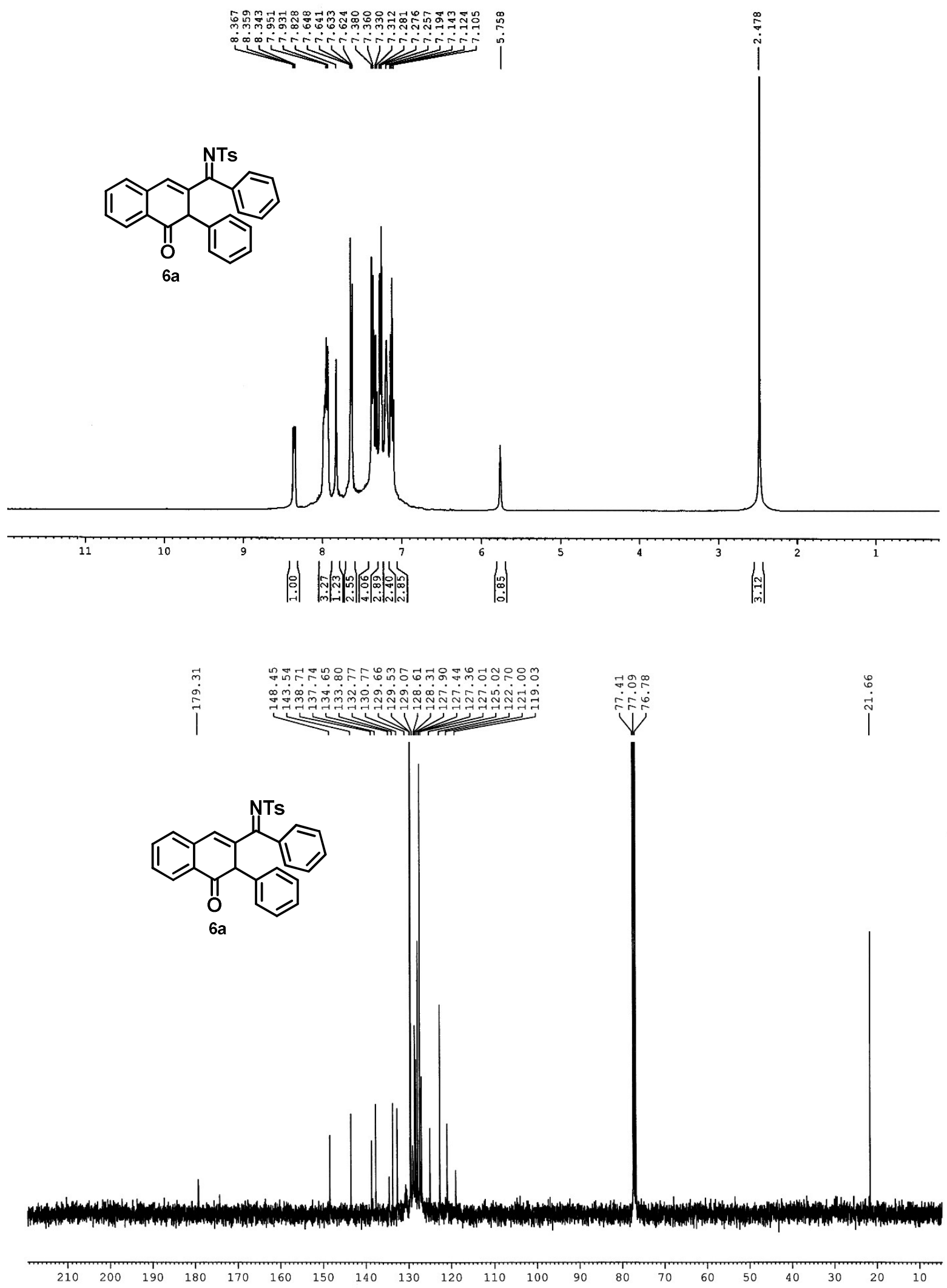


${ }^{1} \mathrm{H}$ NMR (400 MHz) and ${ }^{13} \mathrm{C}$ NMR (100 MHz) spectra of compound $6 \mathrm{~b}$ in $\mathrm{CDCl}_{3}$.
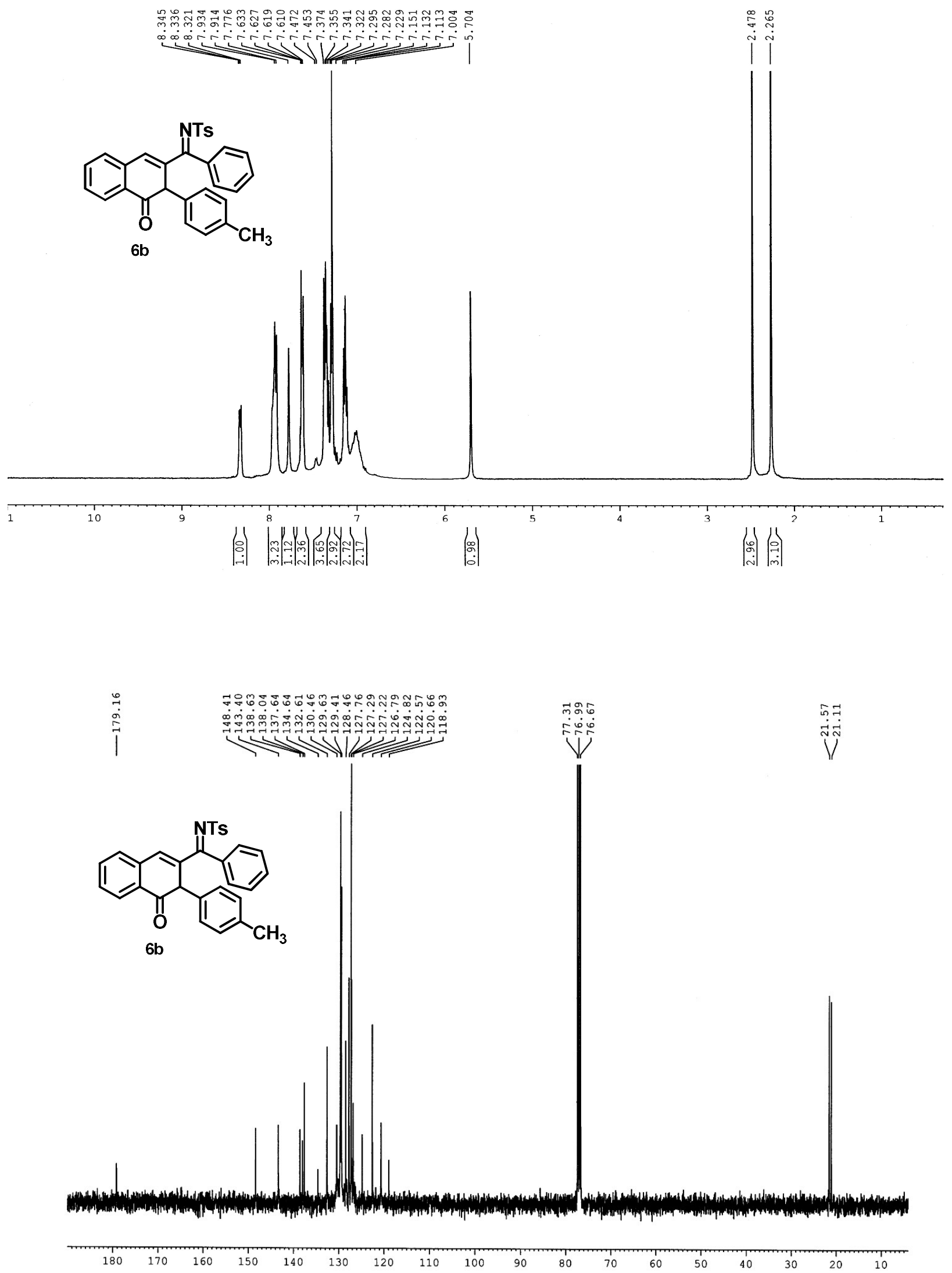
${ }^{1} \mathrm{H}$ NMR (400 MHz) and ${ }^{13} \mathrm{C}$ NMR (100 MHz) spectra of compound 6c in $\mathrm{CDCl}_{3}$.

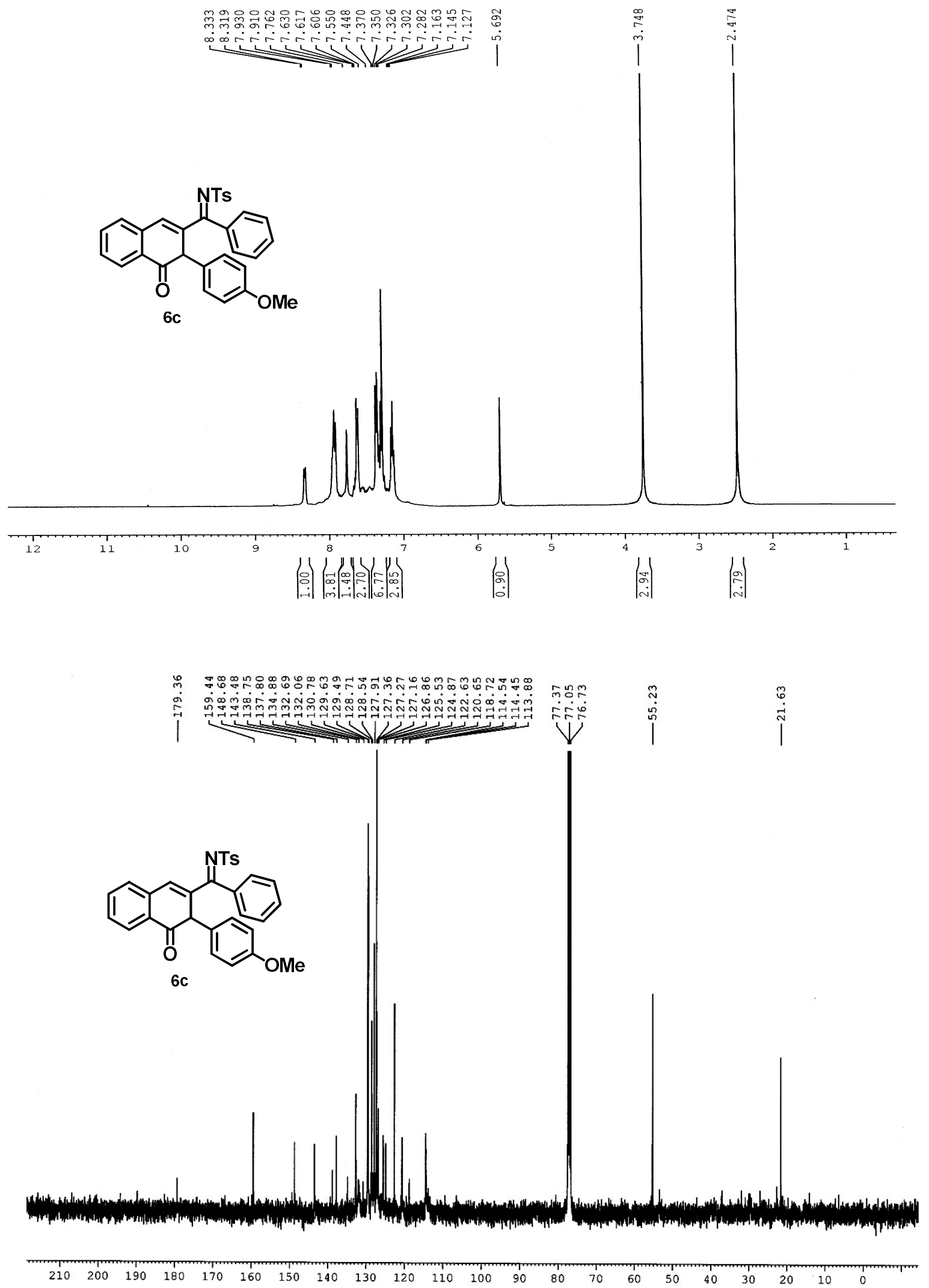


${ }^{1} \mathrm{H}$ NMR (400 MHz) and ${ }^{13} \mathrm{C}$ NMR (100 MHz) spectra of compound $6 \mathrm{~d}$ in $\mathrm{CDCl}_{3}$.
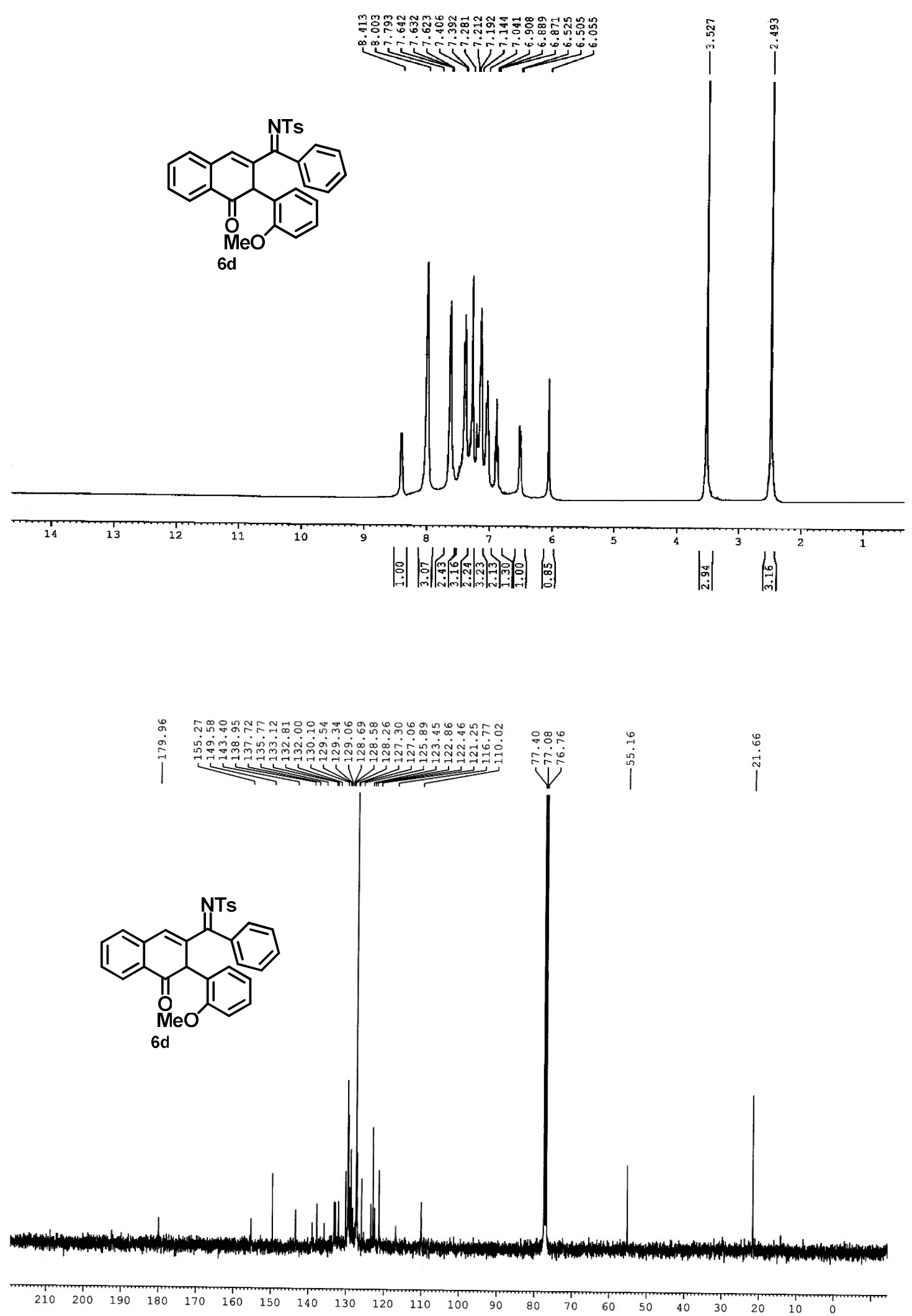
${ }^{1} \mathrm{H}$ NMR (400 MHz) and ${ }^{13} \mathrm{C}$ NMR (100 MHz) spectra of compound $6 \mathrm{e}$ in $\mathrm{CDCl}_{3}$.

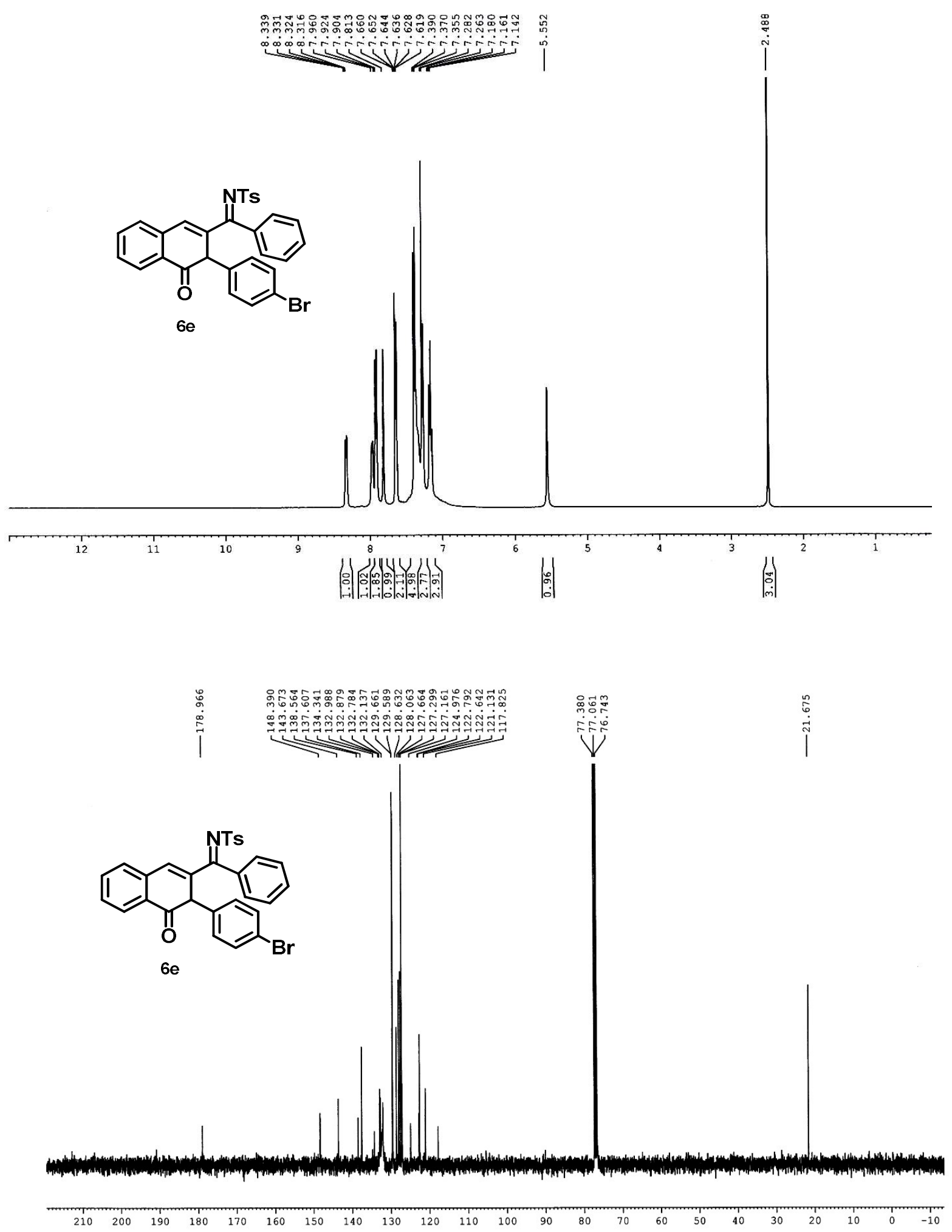


${ }^{1} \mathrm{H}$ NMR (400 MHz) and ${ }^{13} \mathrm{C}$ NMR (100 MHz) spectra of compound $6 \mathrm{f}$ in $\mathrm{CDCl}_{3}$.
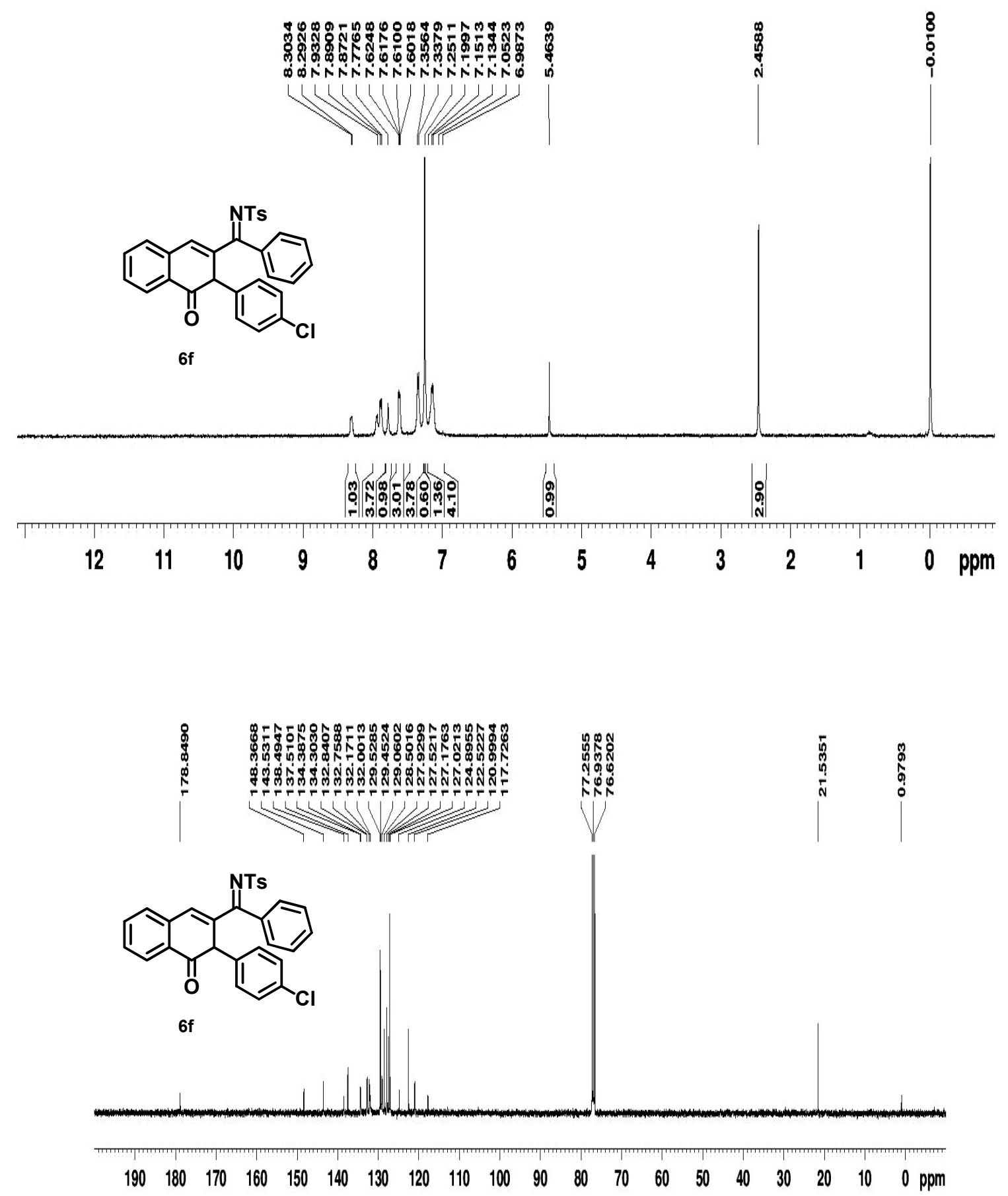
${ }^{1} \mathrm{H}$ NMR (400 MHz) and ${ }^{13} \mathrm{C}$ NMR (75 MHz) spectra of compound $6 \mathrm{~g}$ in $\mathrm{CDCl}_{3}$.
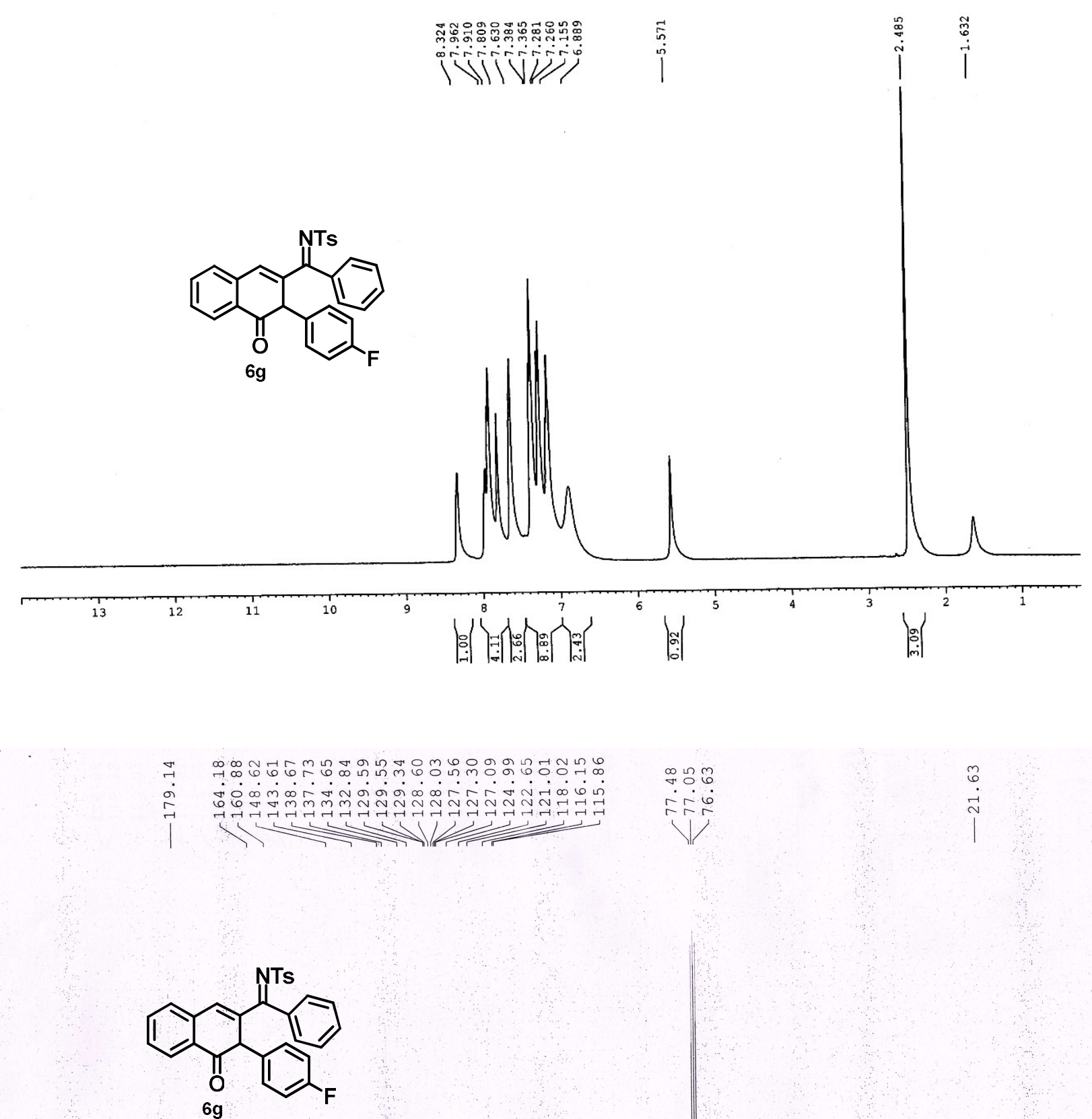

$6 \mathrm{~g}$

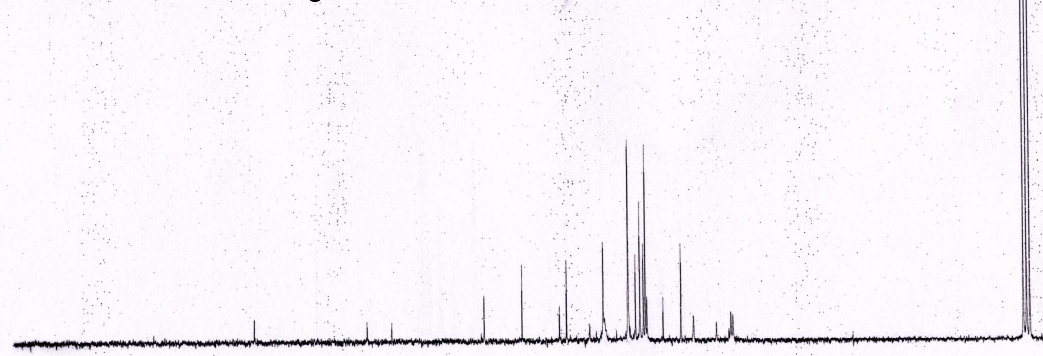

$\begin{array}{llllllllllllllllllllllll}200 & 190 & 180 & 170 & 160 & 150 & 140 & 130 & 120 & 110 & 100 & 90 & 80 & 70 & 60 & 50 & 40 & 30 & 20 & \text { ppm }\end{array}$ 
${ }^{1} \mathrm{H}$ NMR (400 MHz) and ${ }^{13} \mathrm{C}$ NMR (75 MHz) spectra of compound $6 \mathrm{~h}$ in $\mathrm{CDCl}_{3}$.
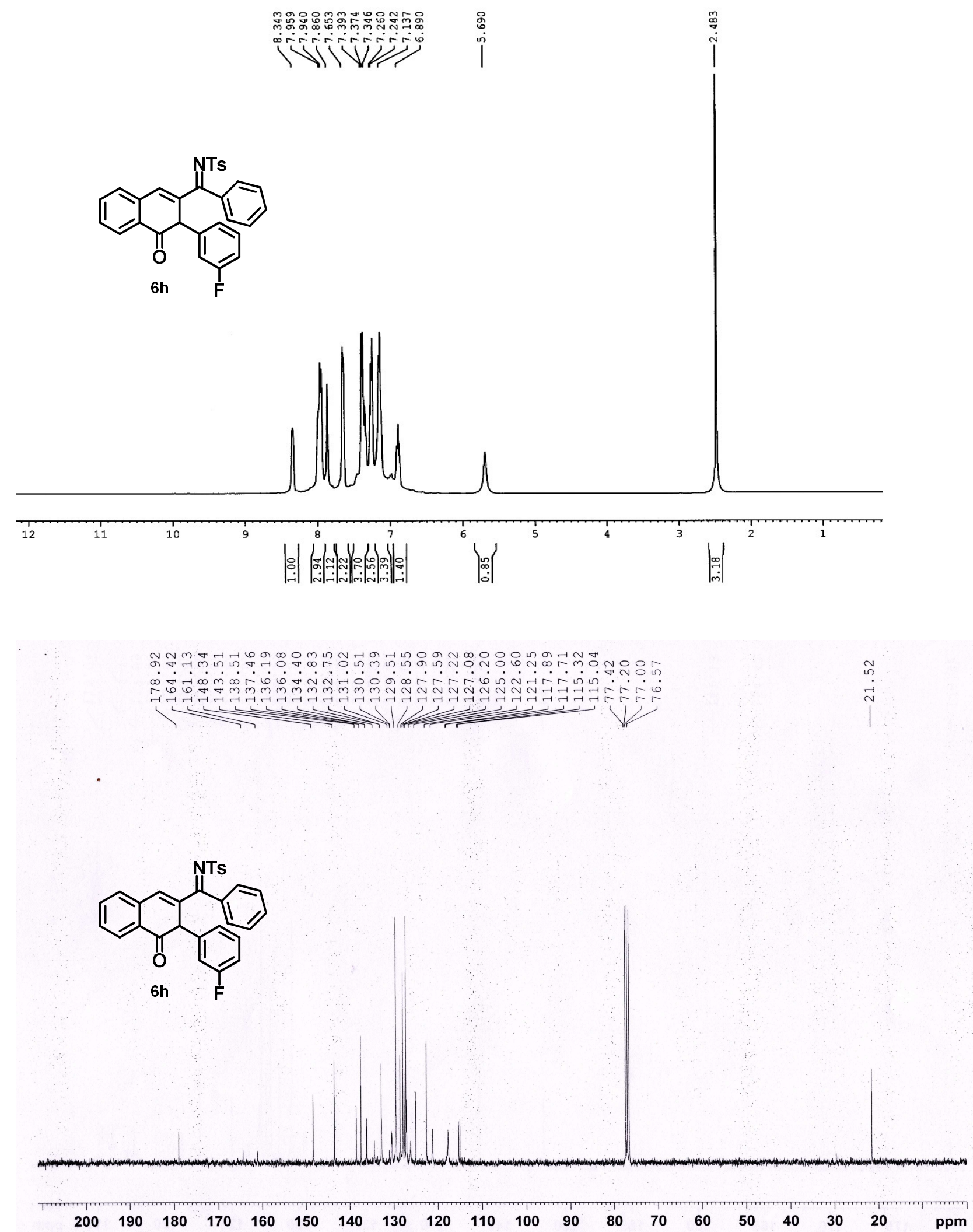
${ }^{1} \mathrm{H}$ NMR (400 MHz) and ${ }^{13} \mathrm{C}$ NMR (100 MHz) spectra of compound $6 \mathrm{i}$ in $\mathrm{CDCl}_{3}$.

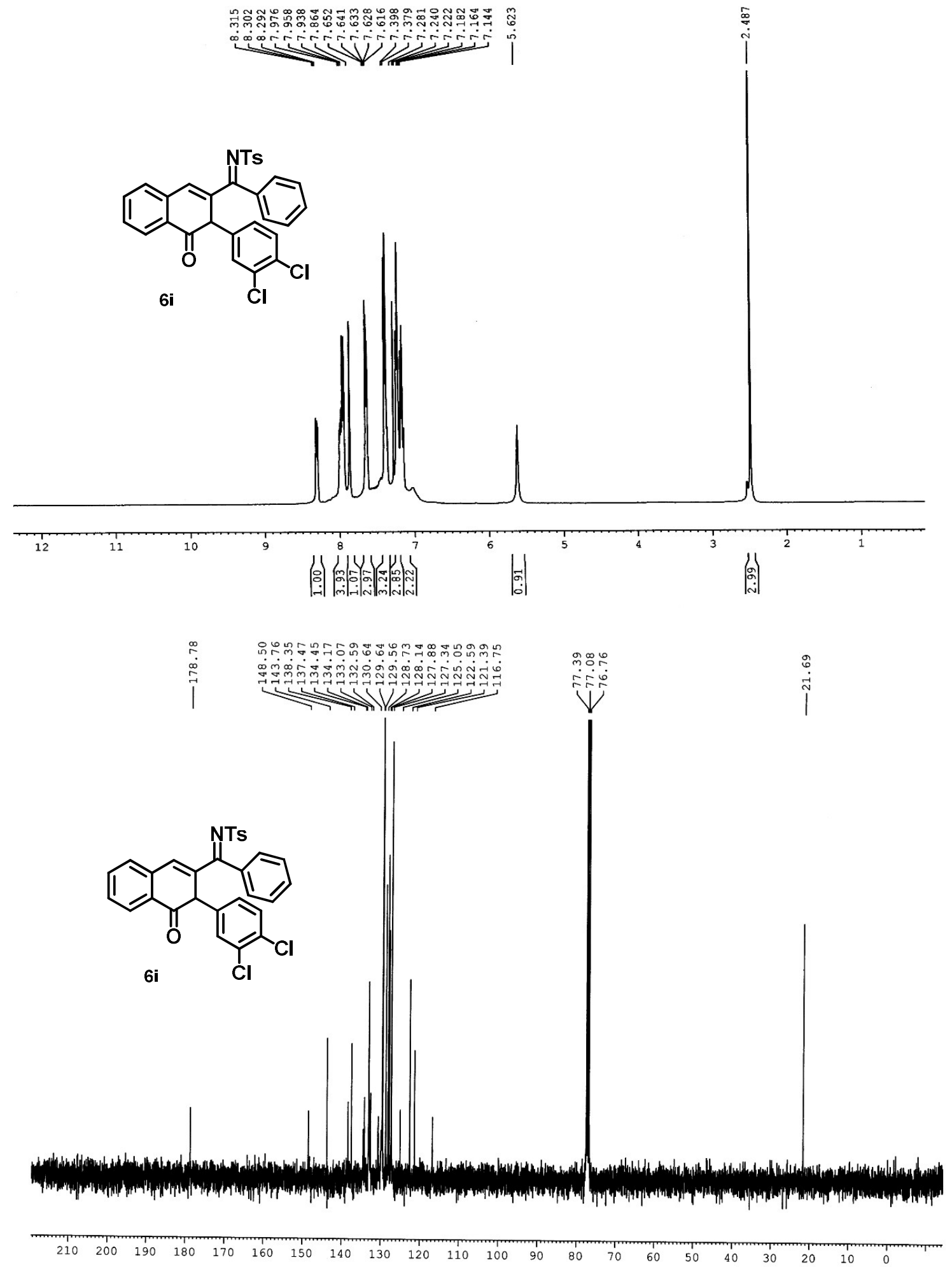


${ }^{1} \mathrm{H}$ NMR (400 MHz) and ${ }^{13} \mathrm{C}$ NMR (100 MHz) spectra of compound 6j in $\mathrm{CDCl}_{3}$.
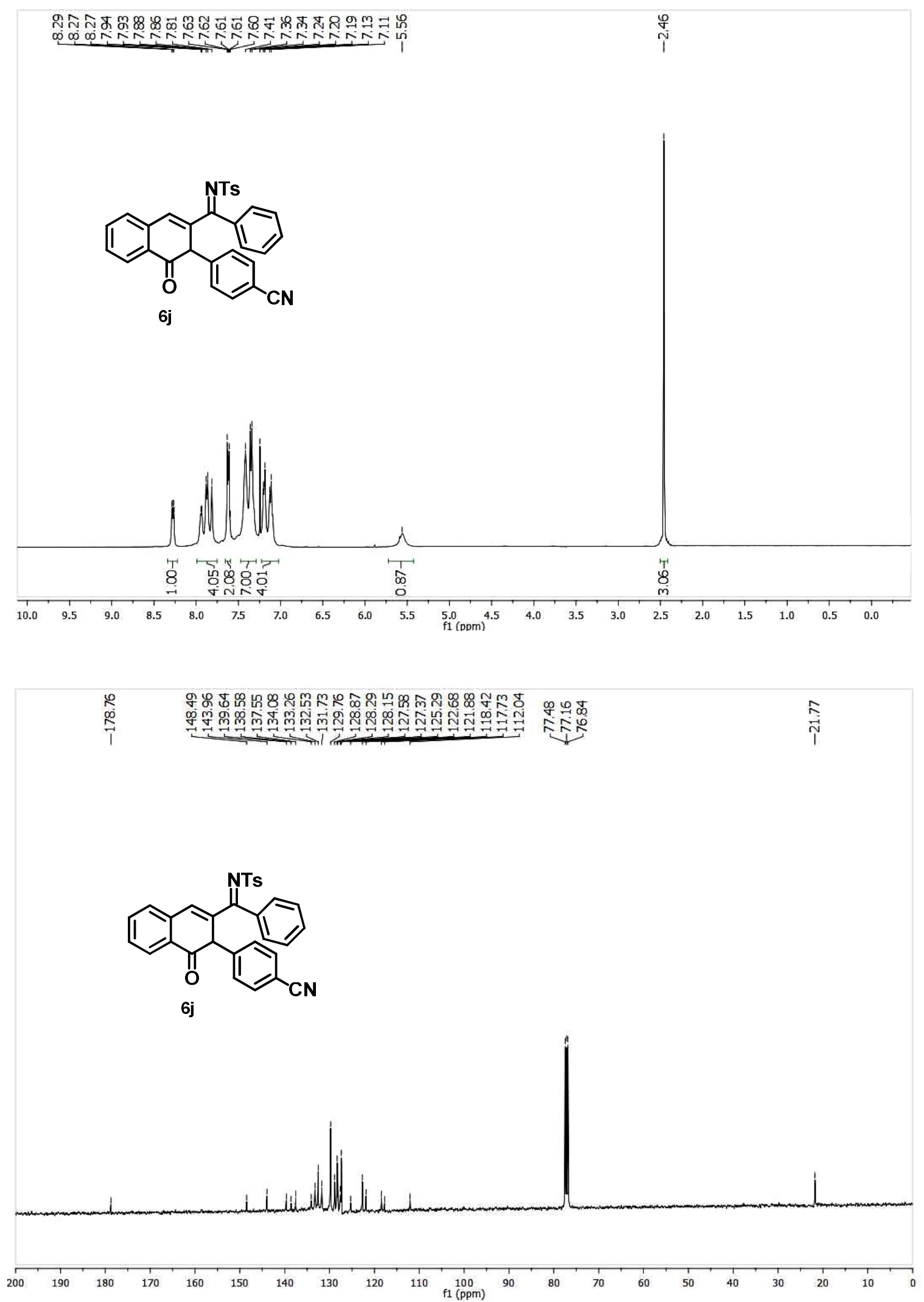
${ }^{1} \mathrm{H}$ NMR (400 MHz) and ${ }^{13} \mathrm{C}$ NMR (100 MHz) spectra of compound 6k in $\mathrm{CDCl}_{3}$.

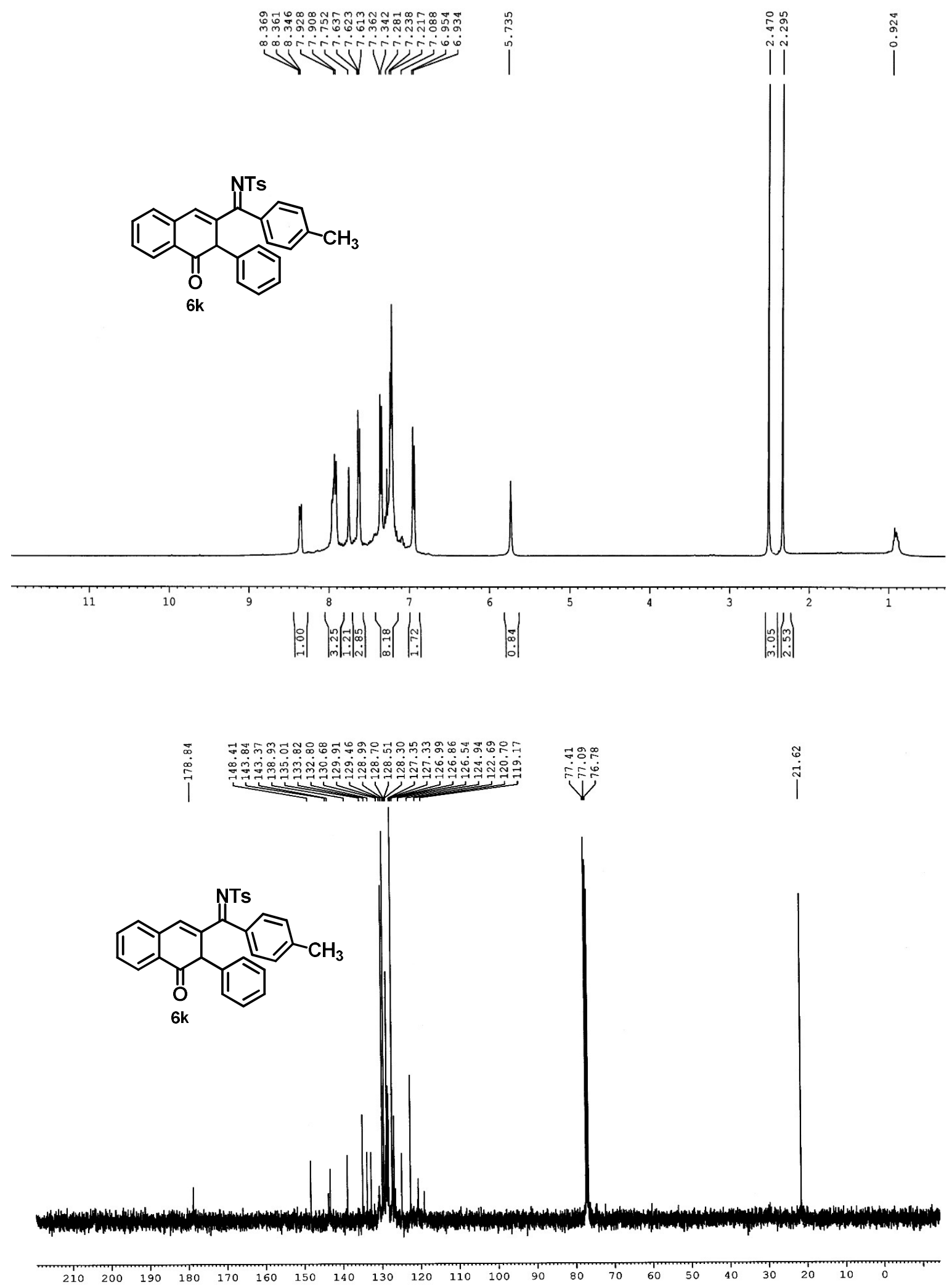


${ }^{1} \mathrm{H}$ NMR (400 MHz) and ${ }^{13} \mathrm{C}$ NMR (100 MHz) spectra of compound $6 \mathrm{I}$ in $\mathrm{CDCl}_{3}$.

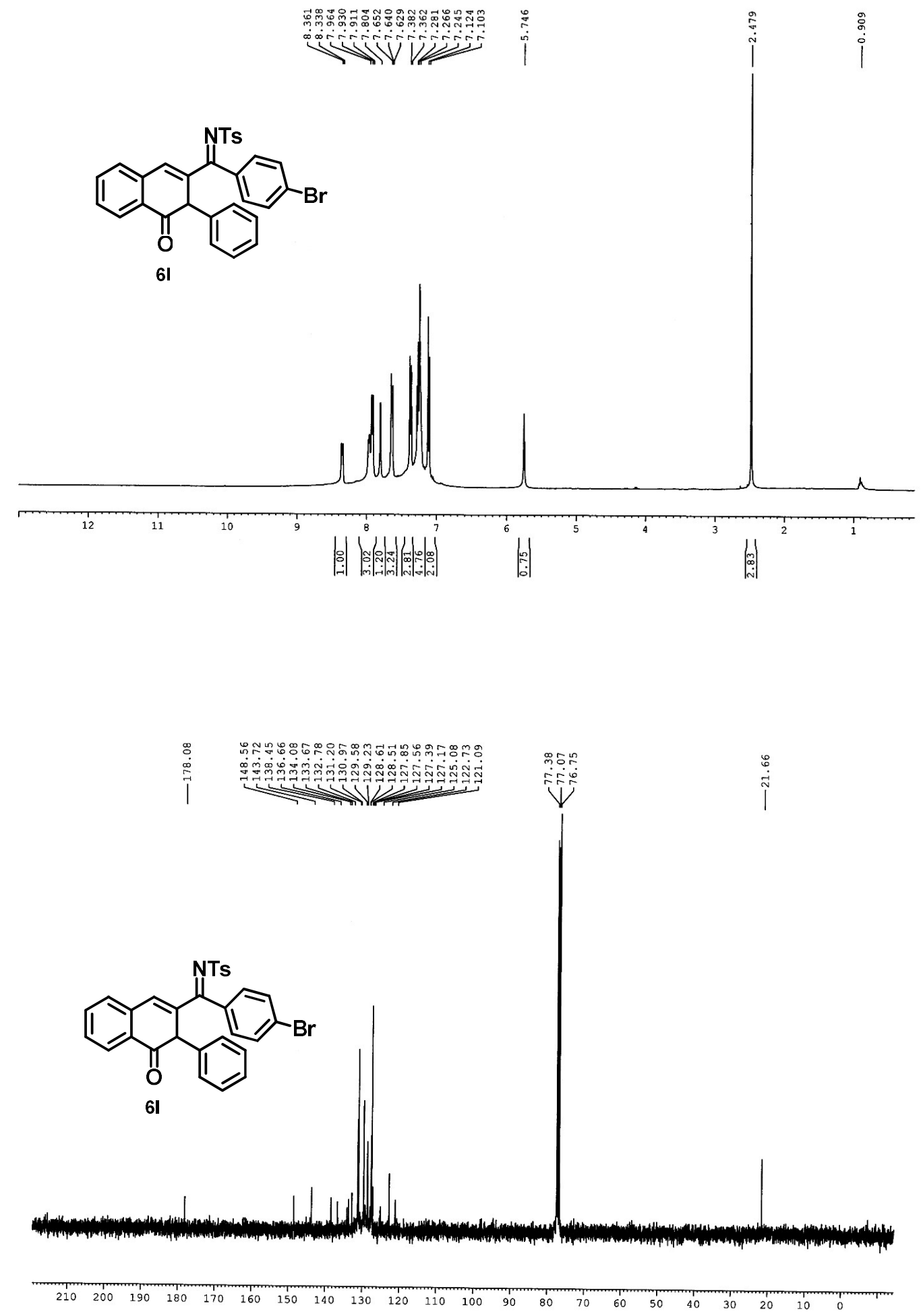


${ }^{1} \mathrm{H}$ NMR (400 MHz) and ${ }^{13} \mathrm{C}$ NMR (100 MHz) spectra of compound $6 \mathrm{~m}$ in $\mathrm{CDCl}_{3}$.

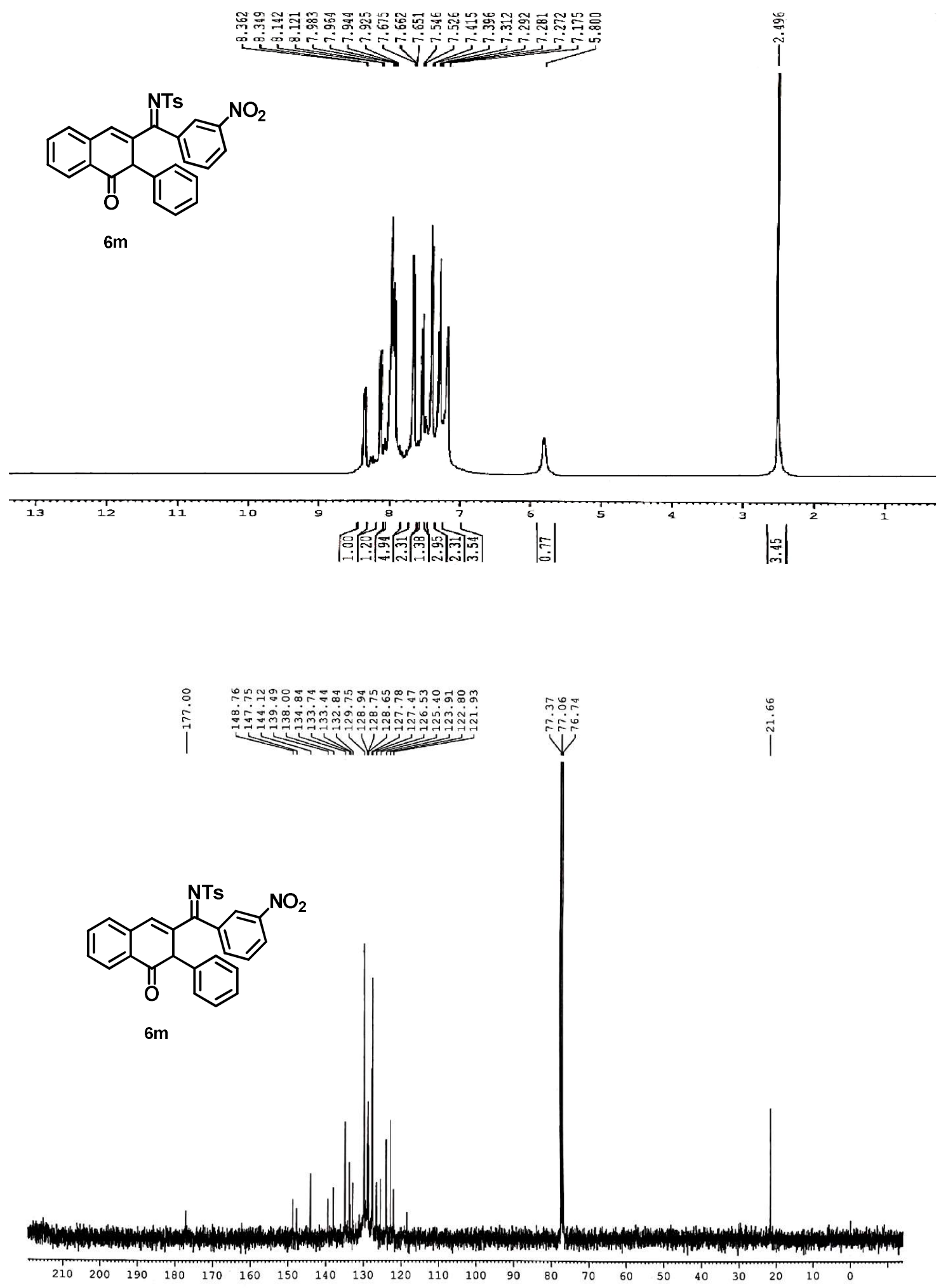


${ }^{1} \mathrm{H}$ NMR (400 MHz) and ${ }^{13} \mathrm{C}$ NMR (100 MHz) spectra of compound $6 \mathrm{n}$ in $\mathrm{CDCl}_{3}$.
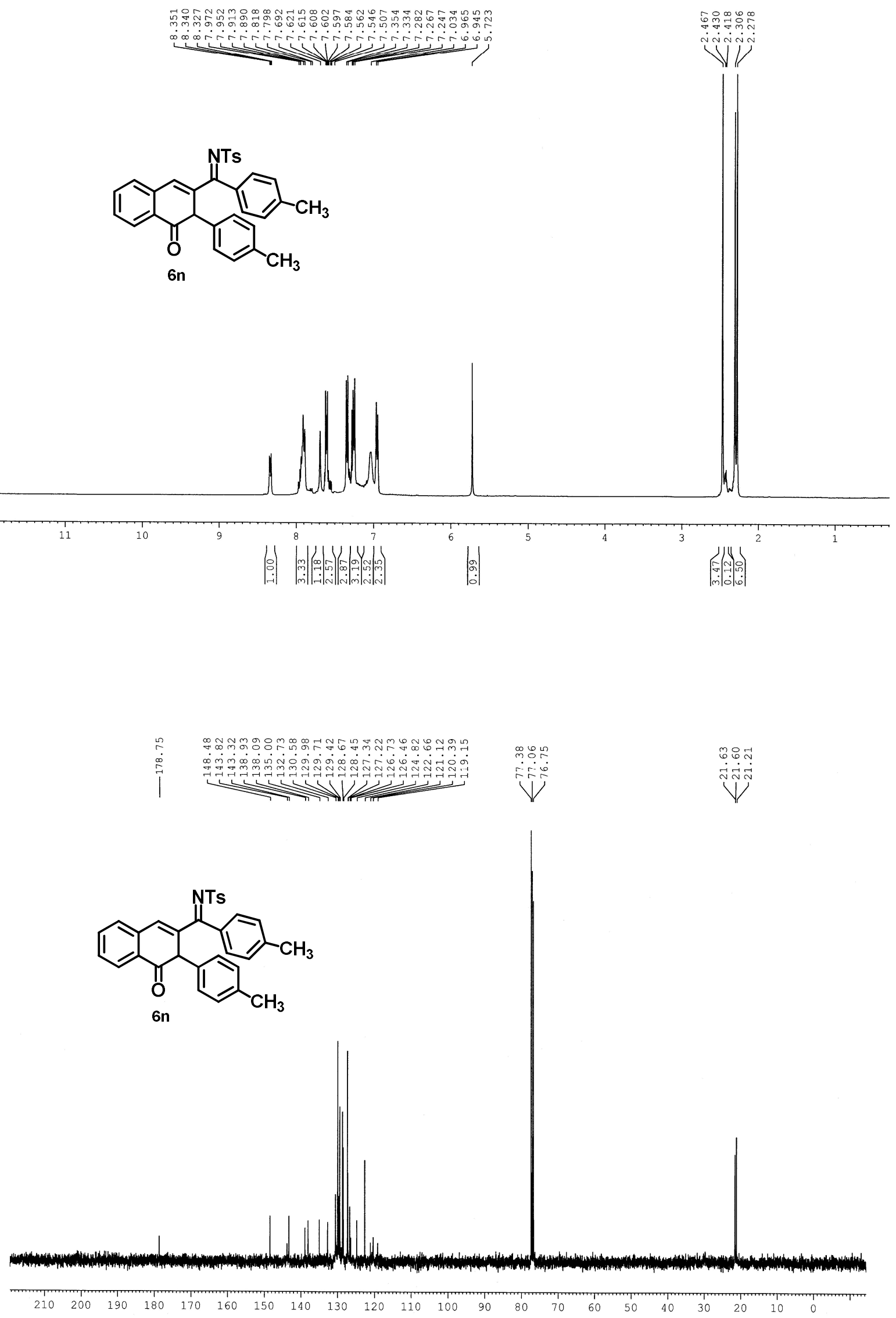

S-36 
${ }^{1} \mathrm{H}$ NMR (400 MHz) and ${ }^{13} \mathrm{C}$ NMR (100 MHz) spectra of compound 60 in $\mathrm{CDCl}_{3}$.

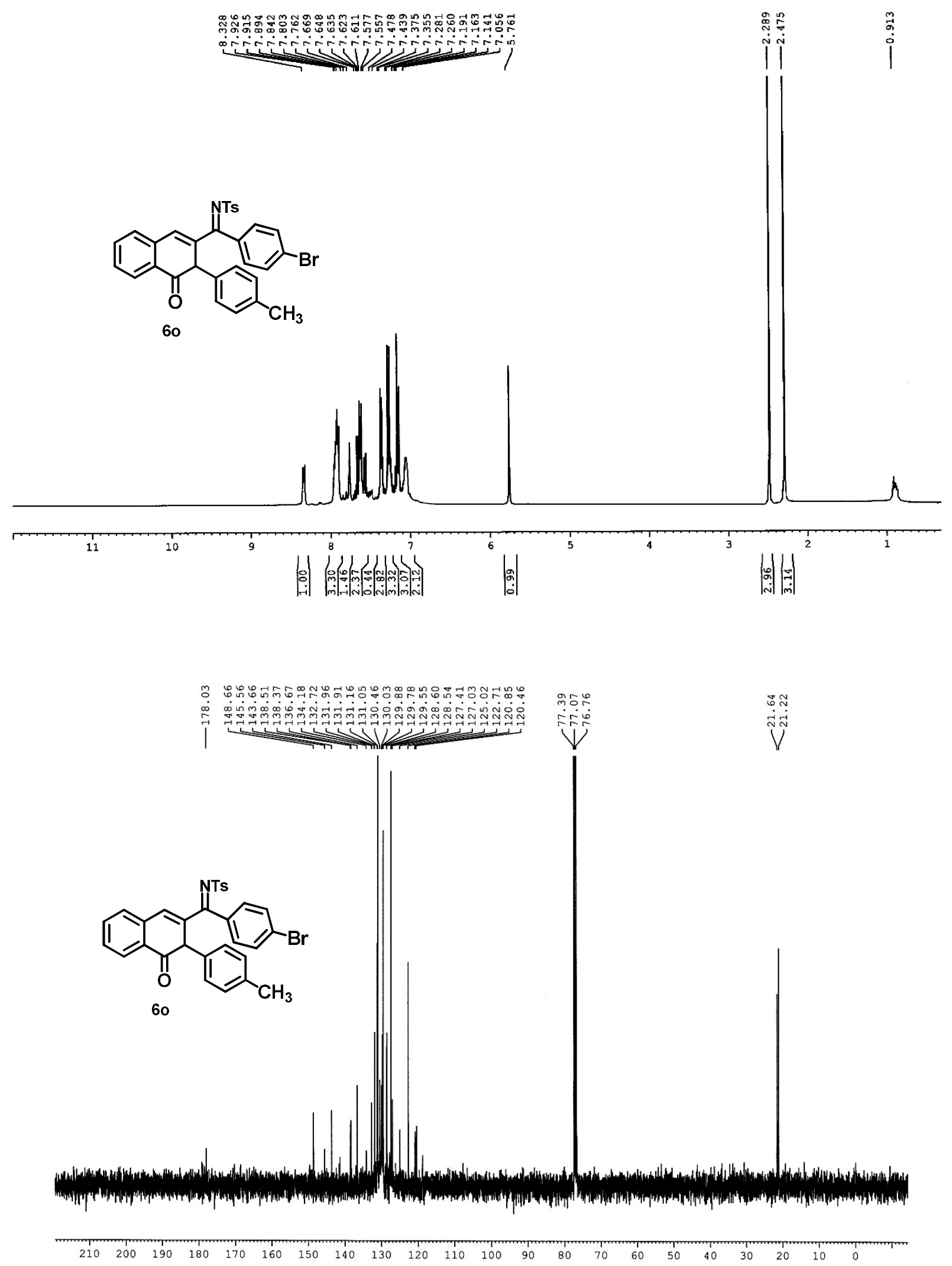


${ }^{1} \mathrm{H}$ NMR (400 MHz) and ${ }^{13} \mathrm{C}$ NMR (100 MHz) spectra of compound $6 \mathrm{p}$ in $\mathrm{CDCl}_{3}$.

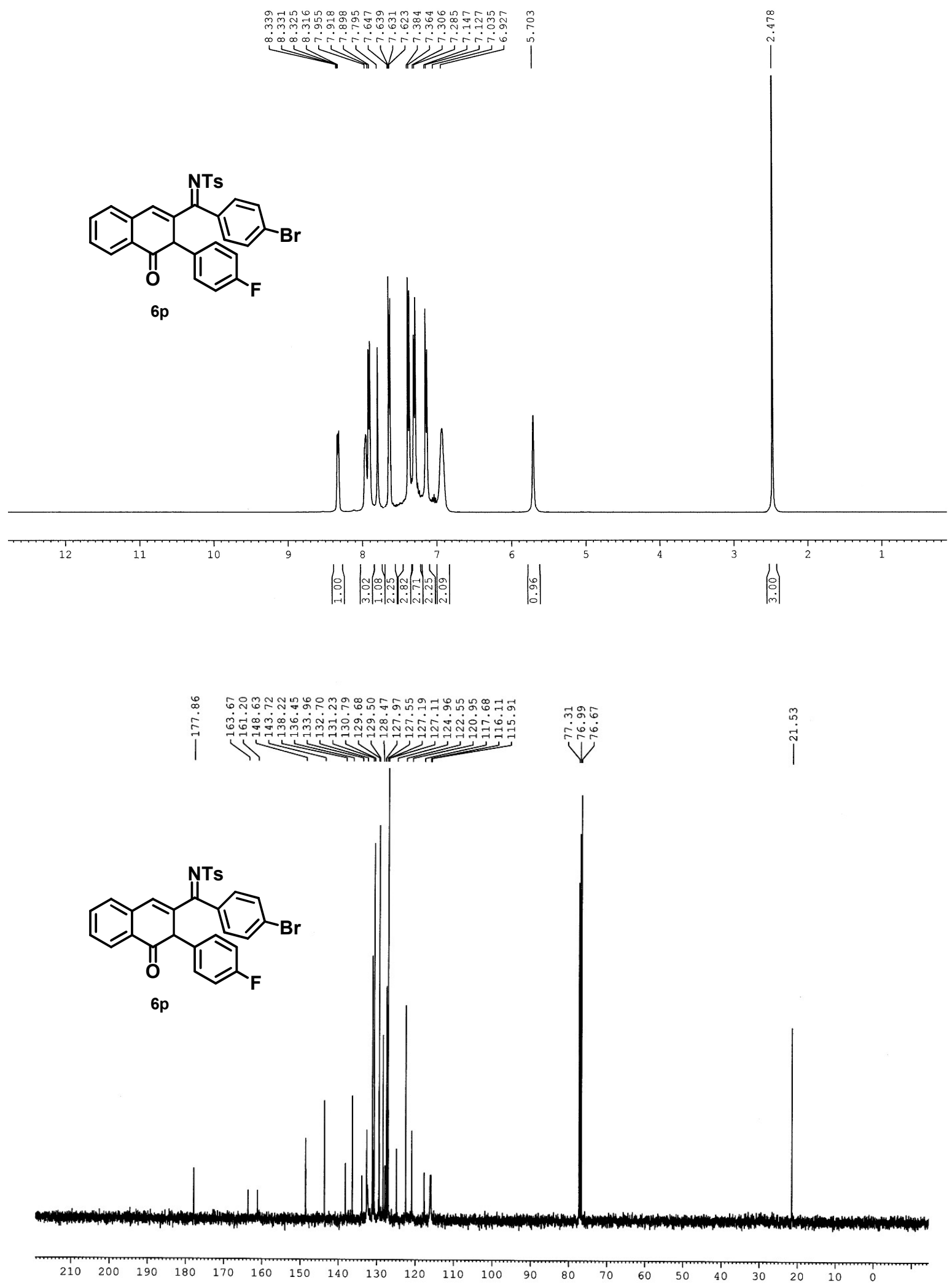


${ }^{1} \mathrm{H}$ NMR (400 MHz) and ${ }^{13} \mathrm{C}$ NMR (100 MHz) spectra of compound $6 q$ in $\mathrm{CDCl}_{3}$.
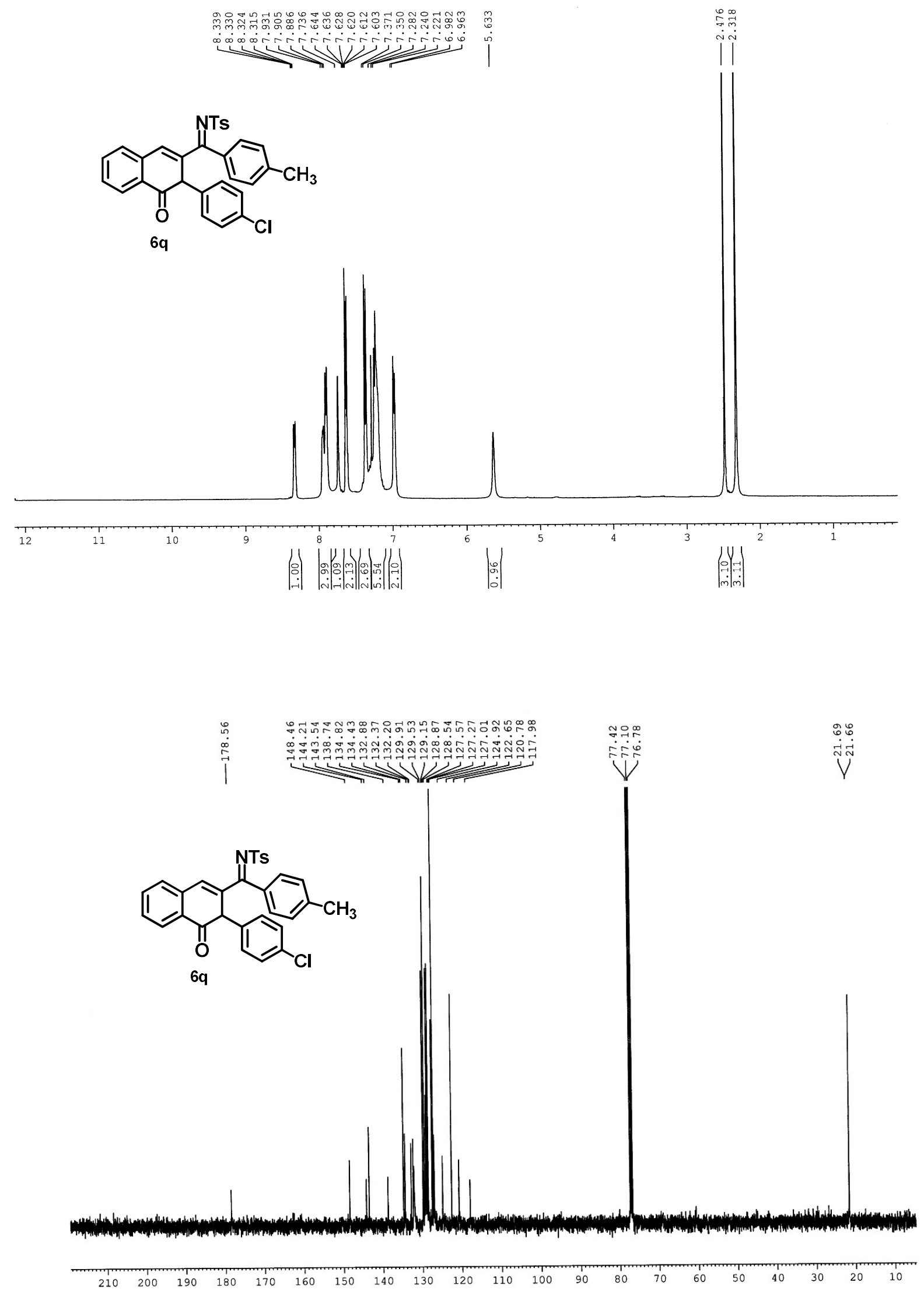
${ }^{1} \mathrm{H}$ NMR (400 MHz) and ${ }^{13} \mathrm{C}$ NMR (100 MHz) spectra of compound $6 \mathrm{r}$ in $\mathrm{CDCl}_{3}$.

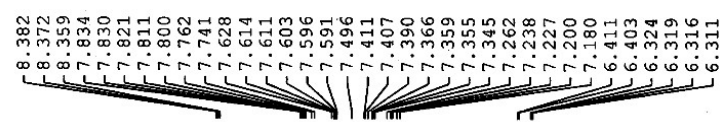<smiles>C[N+]=C(C1=Cc2ccccc2C(=O)C1c1ccco1)c1ccc(Br)cc1</smiles>

$6 r$
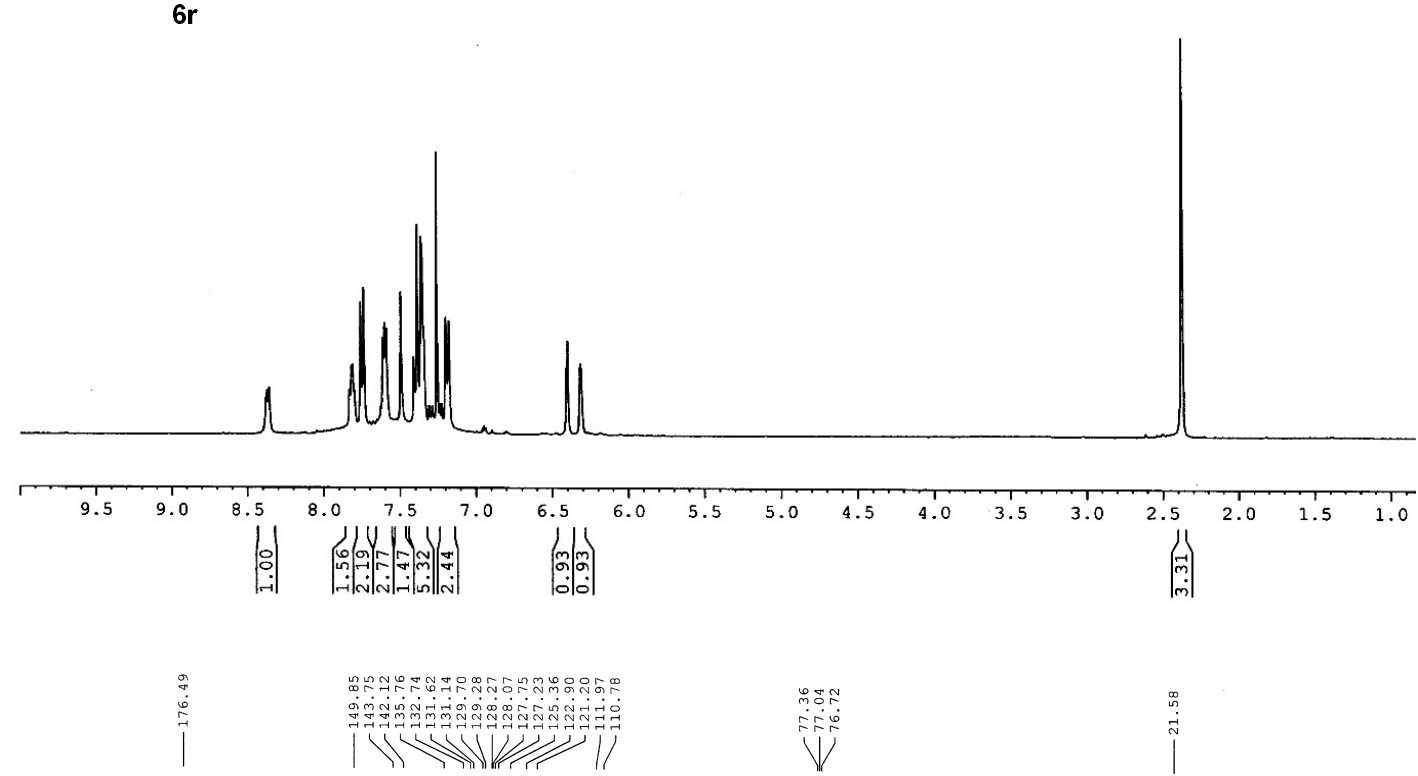<smiles></smiles>

$6 r$

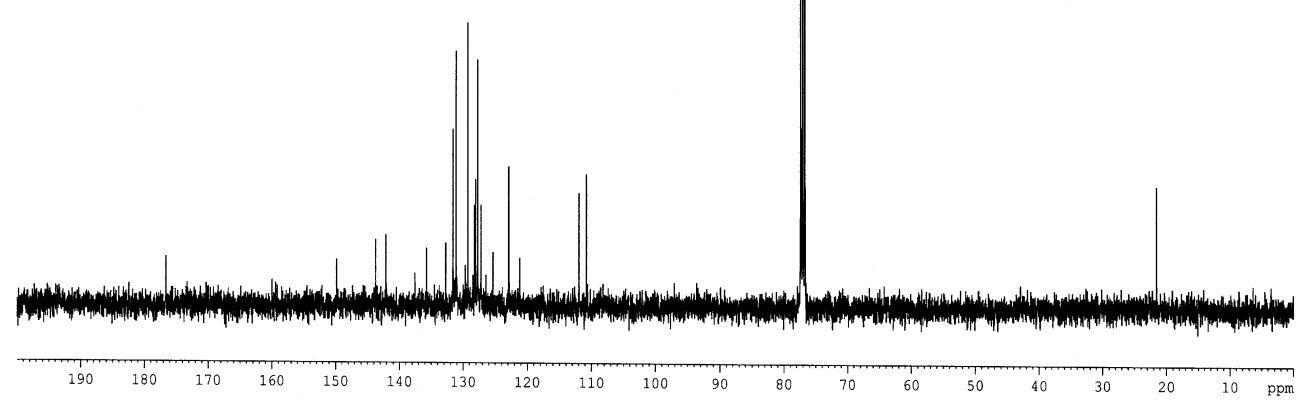


${ }^{1} \mathrm{H}$ NMR (400 MHz) and ${ }^{13} \mathrm{C}$ NMR (100 MHz) spectra of compound 6 s in $\mathrm{CDCl}_{3}$.

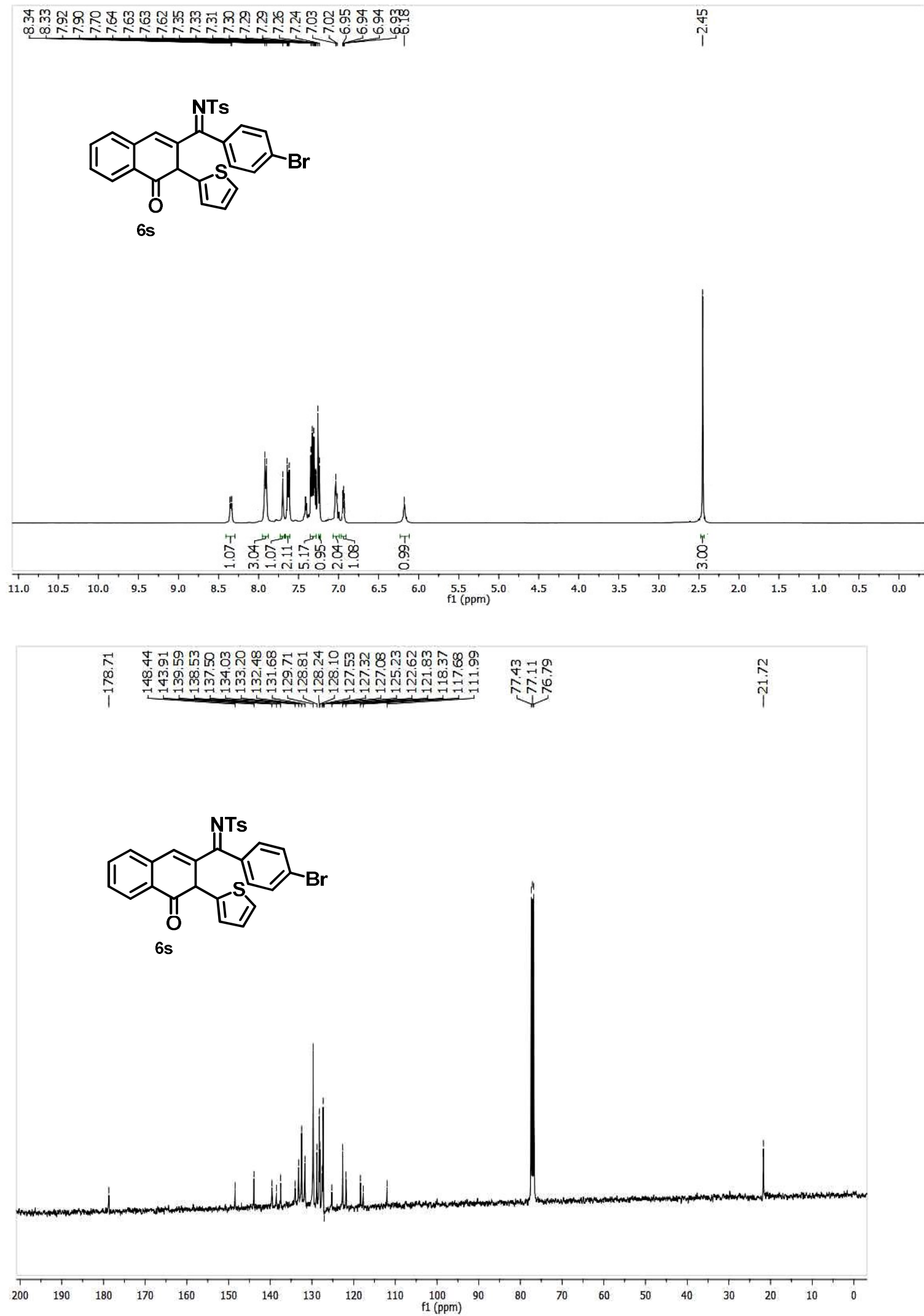


${ }^{1} \mathrm{H}$ NMR (300 MHz) and ${ }^{13} \mathrm{C}$ NMR (75 MHz) spectra of compound $6 \mathrm{t}$ in $\mathrm{CDCl}_{3}$.

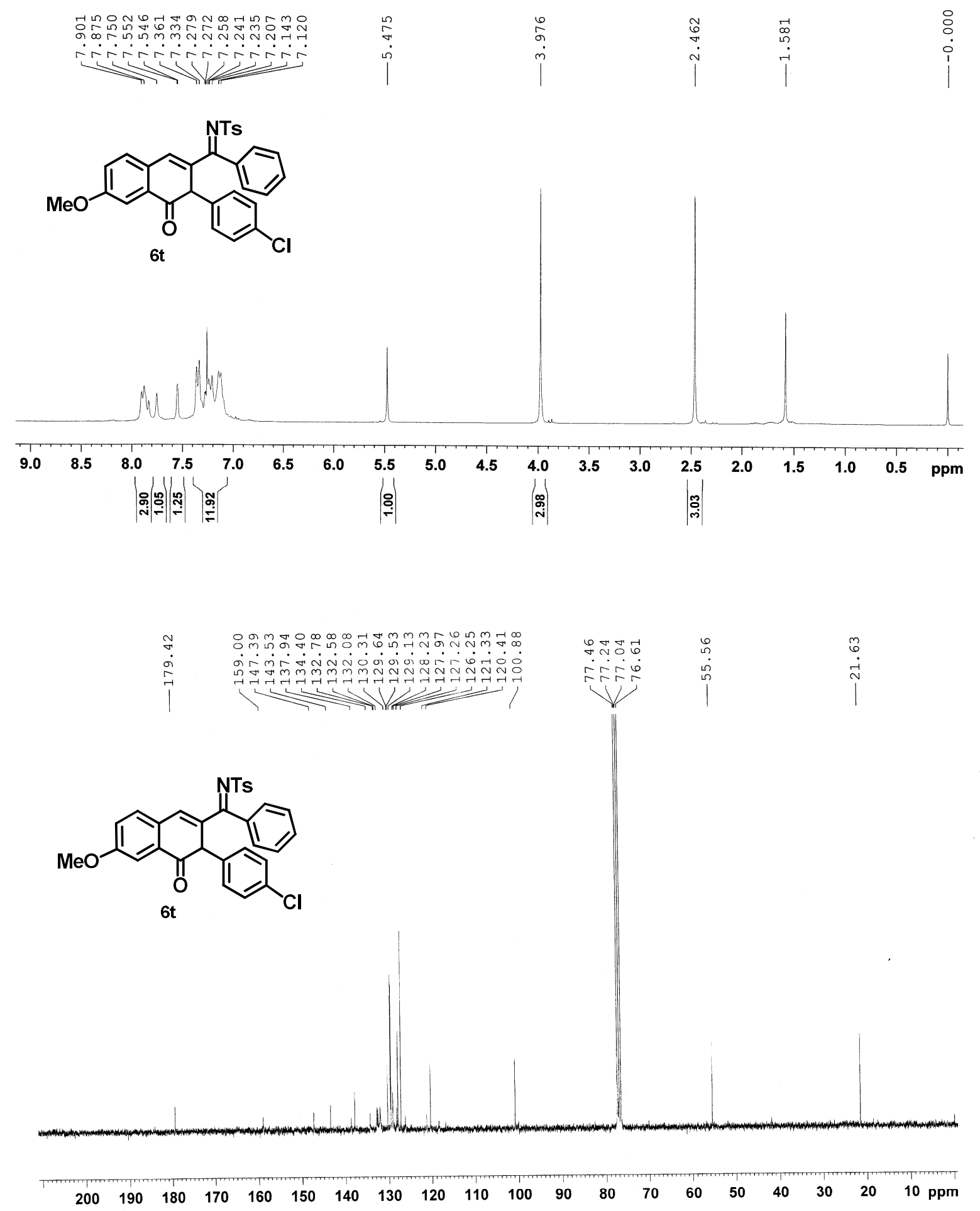


Section VII: ${ }^{1} \mathrm{H}$ and ${ }^{13} \mathrm{C}$-NMR spectra of substituted isoquinoline derivatives

${ }^{1} \mathrm{H}$ NMR (400 MHz) and ${ }^{13} \mathrm{C}$ NMR (100 MHz) spectra of compound 9a in $\mathrm{CDCl}_{3}$.
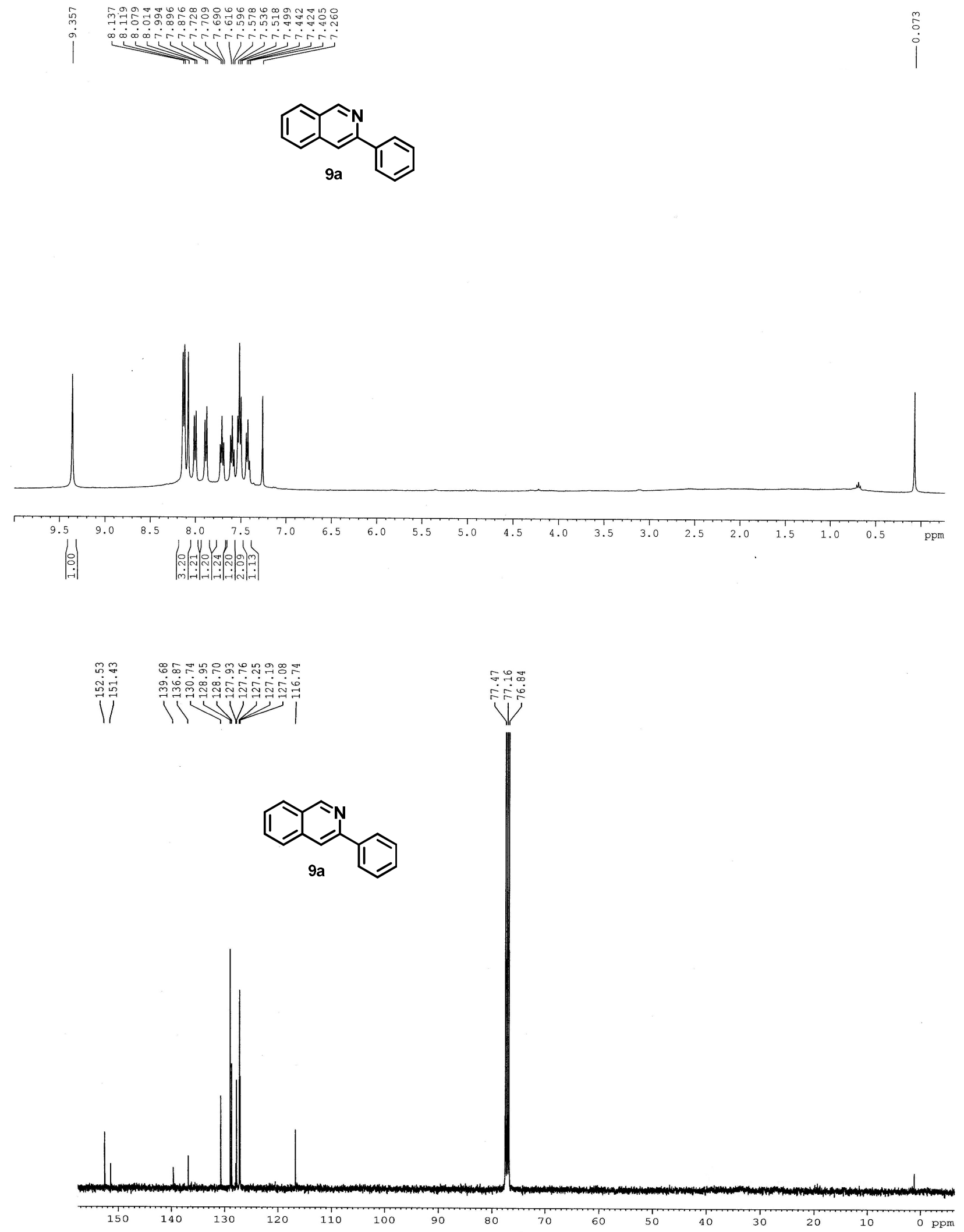
${ }^{1} \mathrm{H}$ NMR (400 MHz) and ${ }^{13} \mathrm{C}$ NMR (100 MHz) spectra of compound 9b in $\mathrm{CDCl}_{3}$.
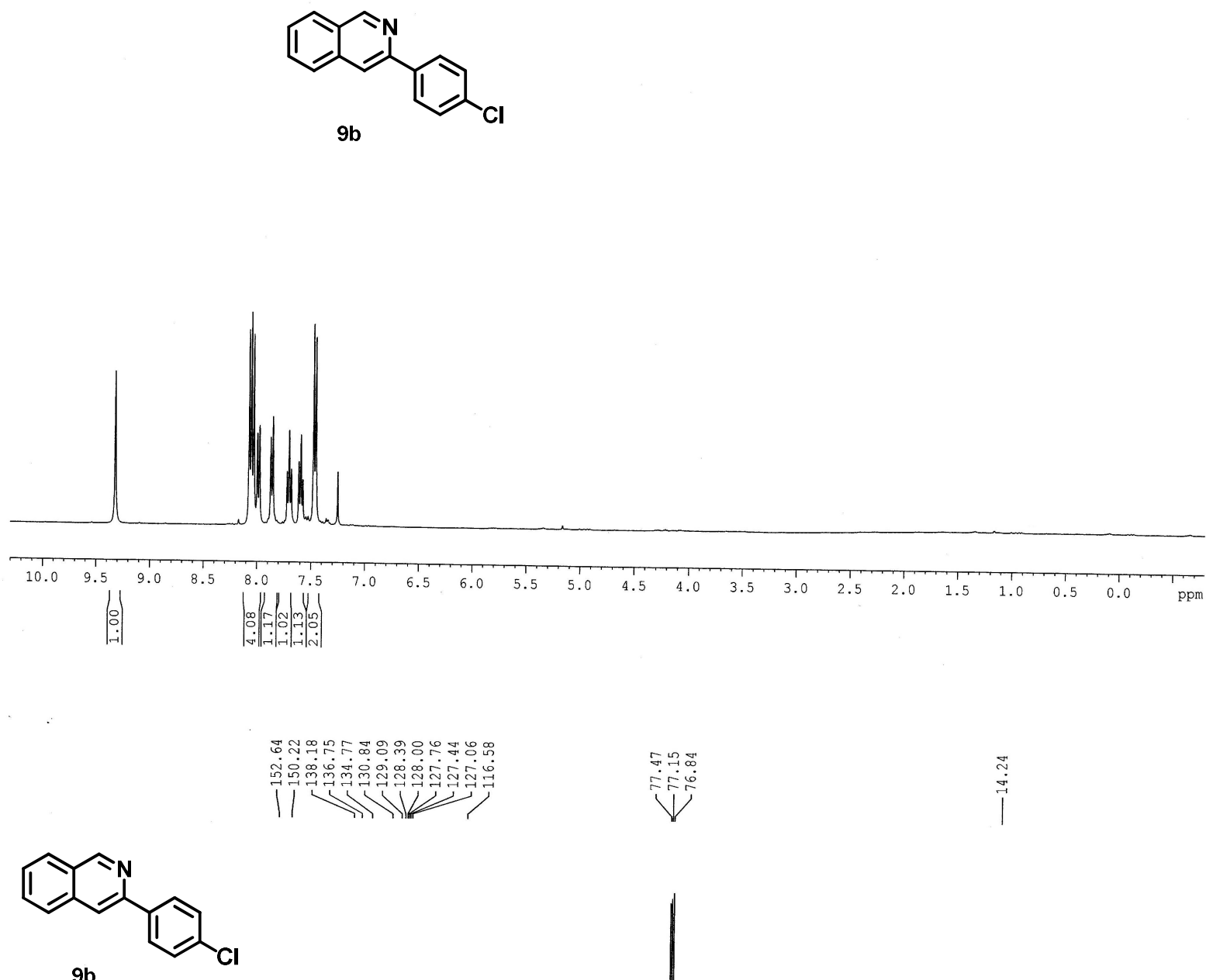

$9 b$

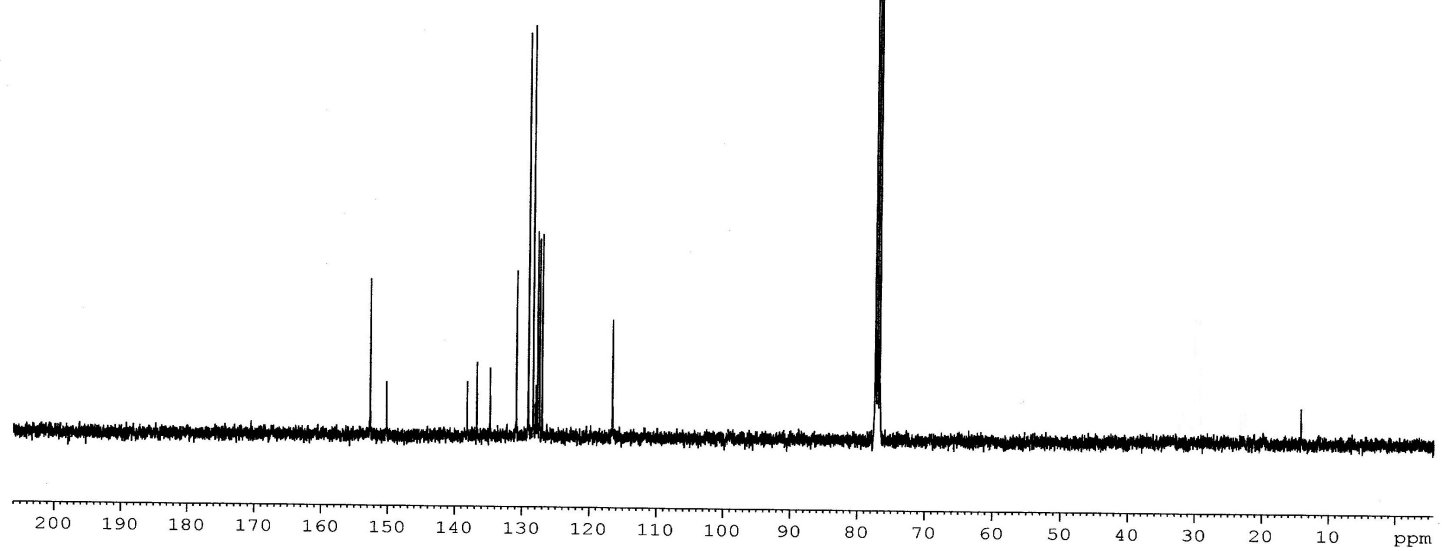


${ }^{1} \mathrm{H}$ NMR (400 MHz) and ${ }^{13} \mathrm{C}$ NMR (100 MHz) spectra of compound 9c in $\mathrm{CDCl}_{3}$.
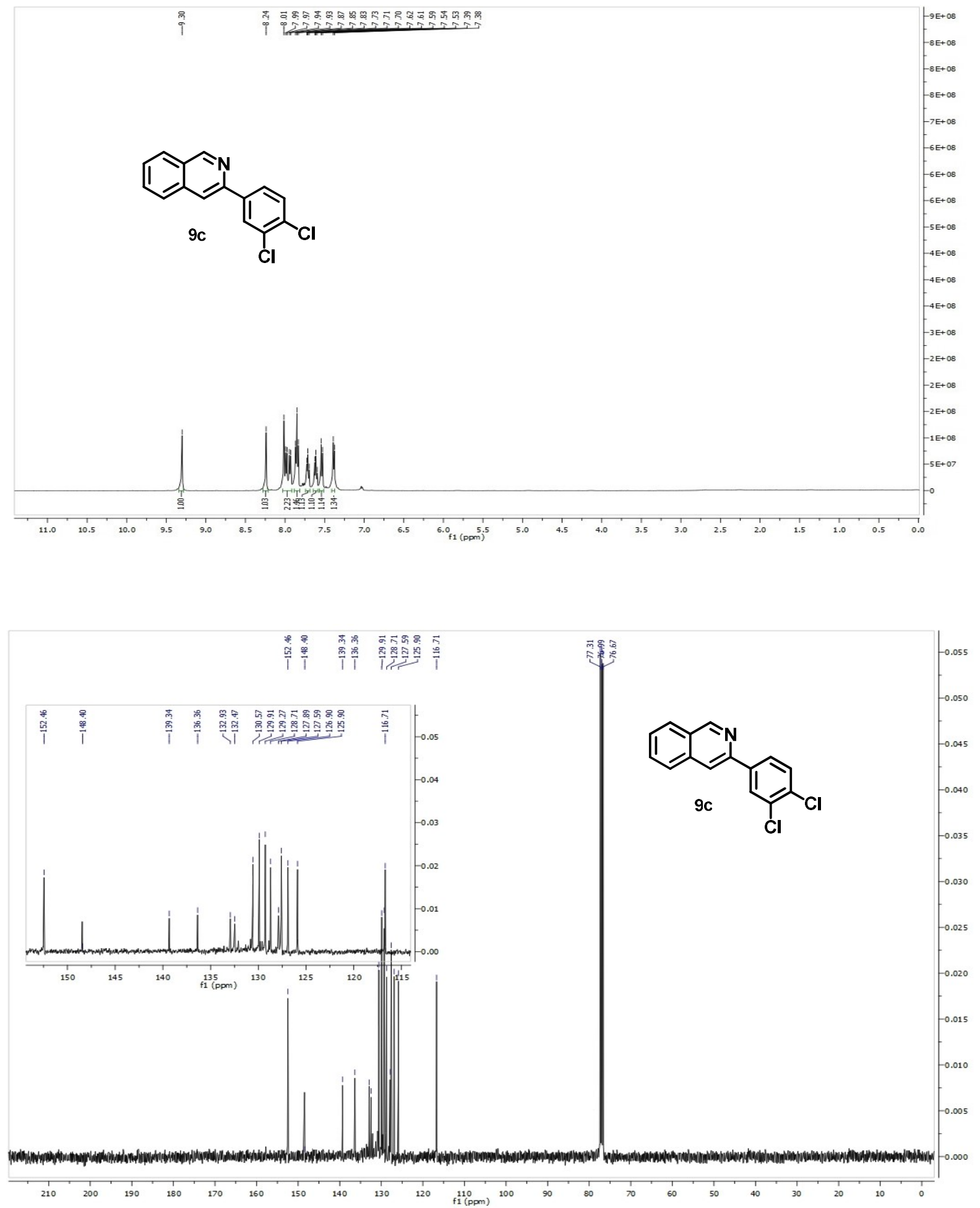
${ }^{1} \mathrm{H}$ NMR (400 MHz) and ${ }^{13} \mathrm{C}$ NMR (100 MHz) spectra of compound 9d in $\mathrm{CDCl}_{3}$.
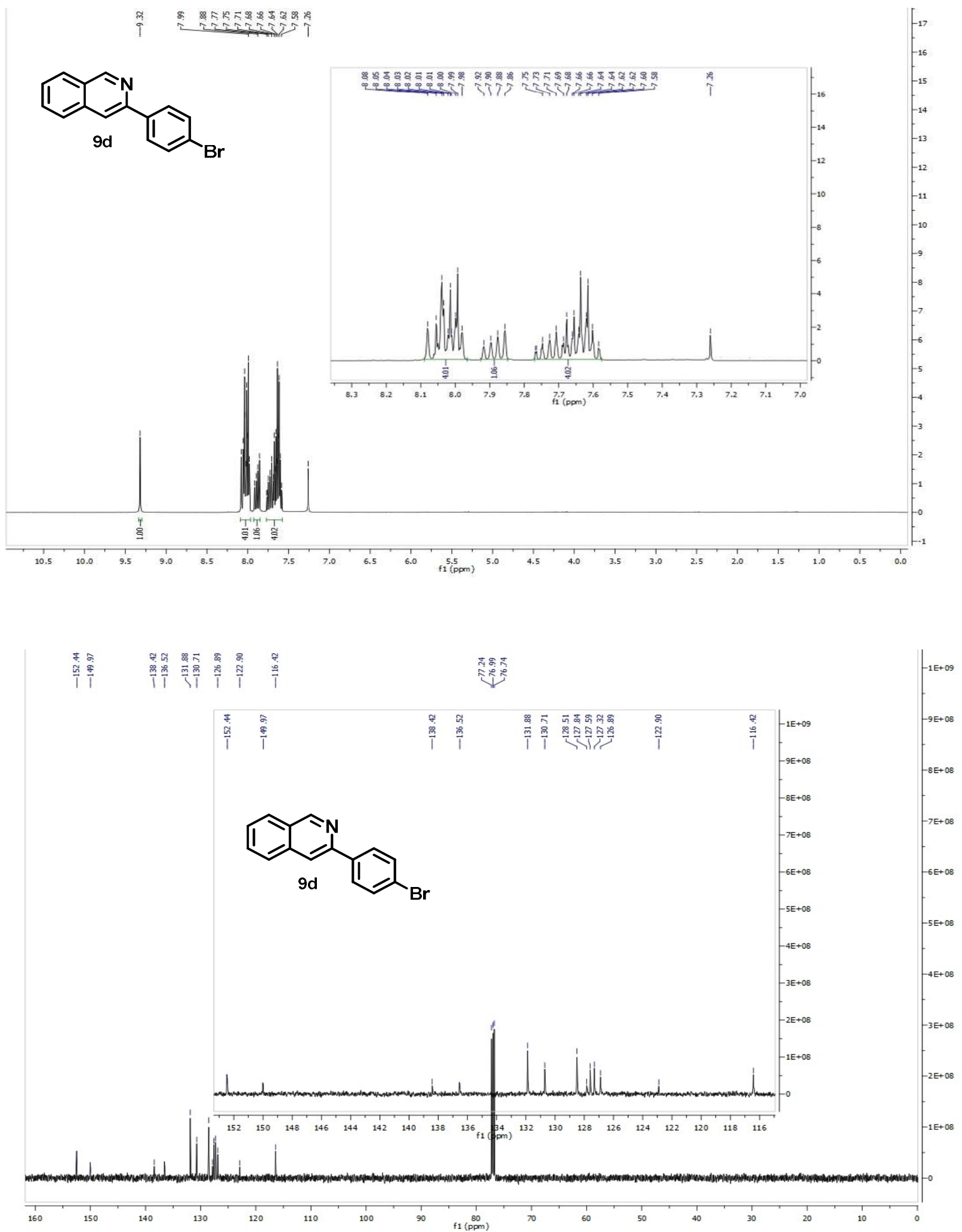
${ }^{1} \mathrm{H}$ NMR (300 MHz) and ${ }^{13} \mathrm{C}$ NMR (75 MHz) spectra of compound ge in $\mathrm{CDCl}_{3}$.
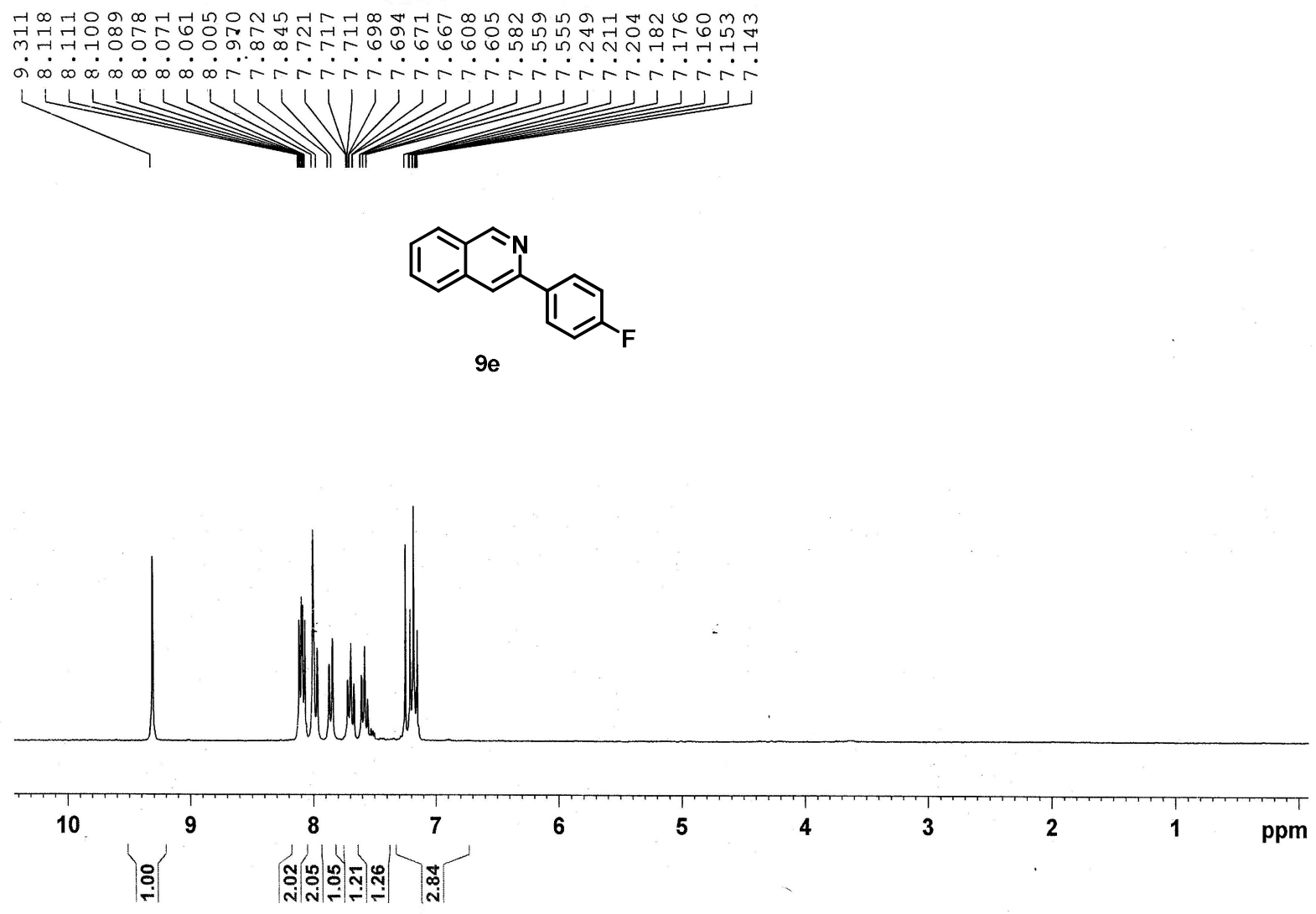<smiles>Fc1ccc(-c2cc3ccccc3cn2)cc1</smiles>
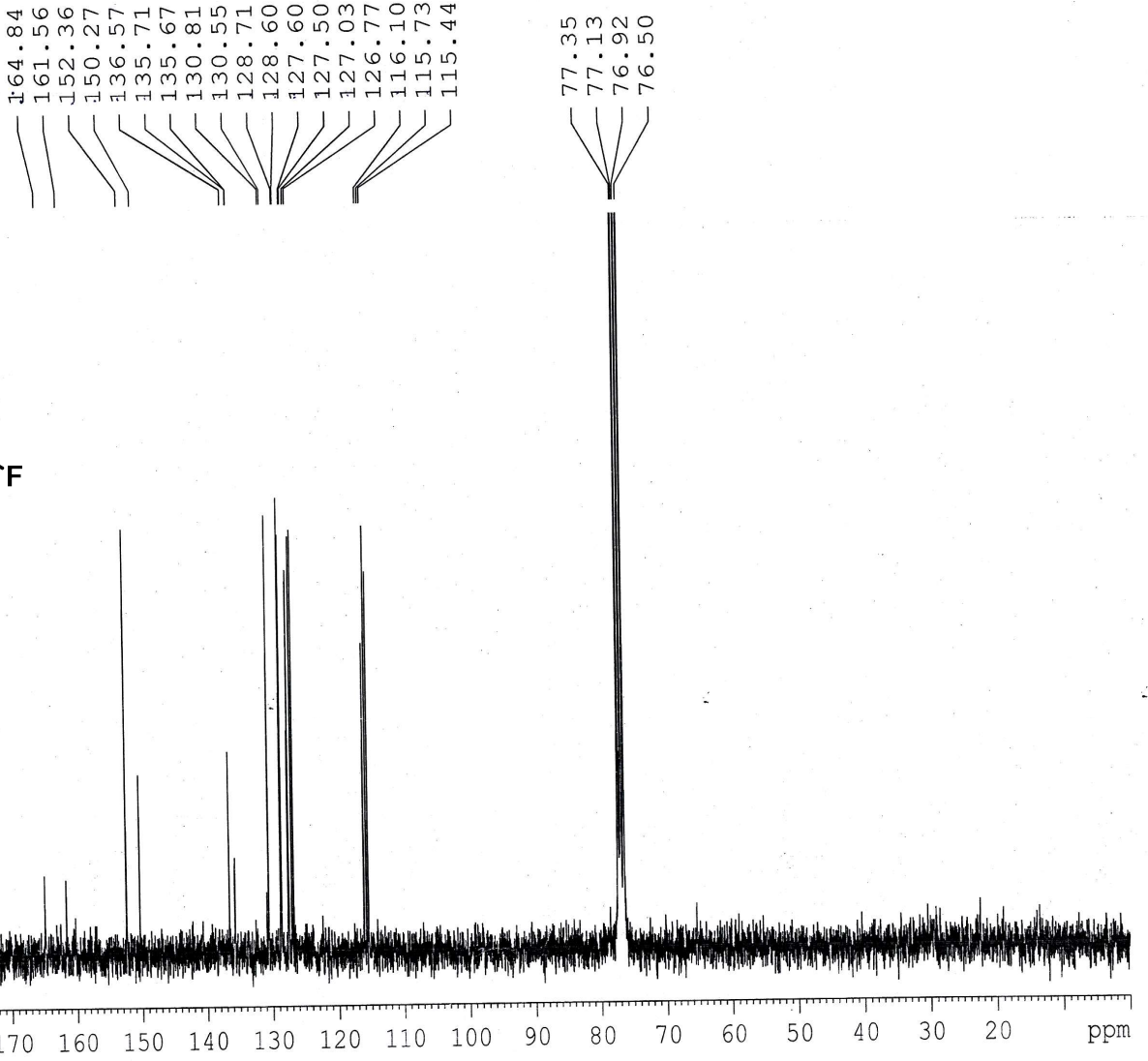
${ }^{1} \mathrm{H}$ NMR (400 MHz) and ${ }^{13} \mathrm{C}$ NMR (75 MHz) spectra of compound 9 in $\mathrm{CDCl}_{3}$.
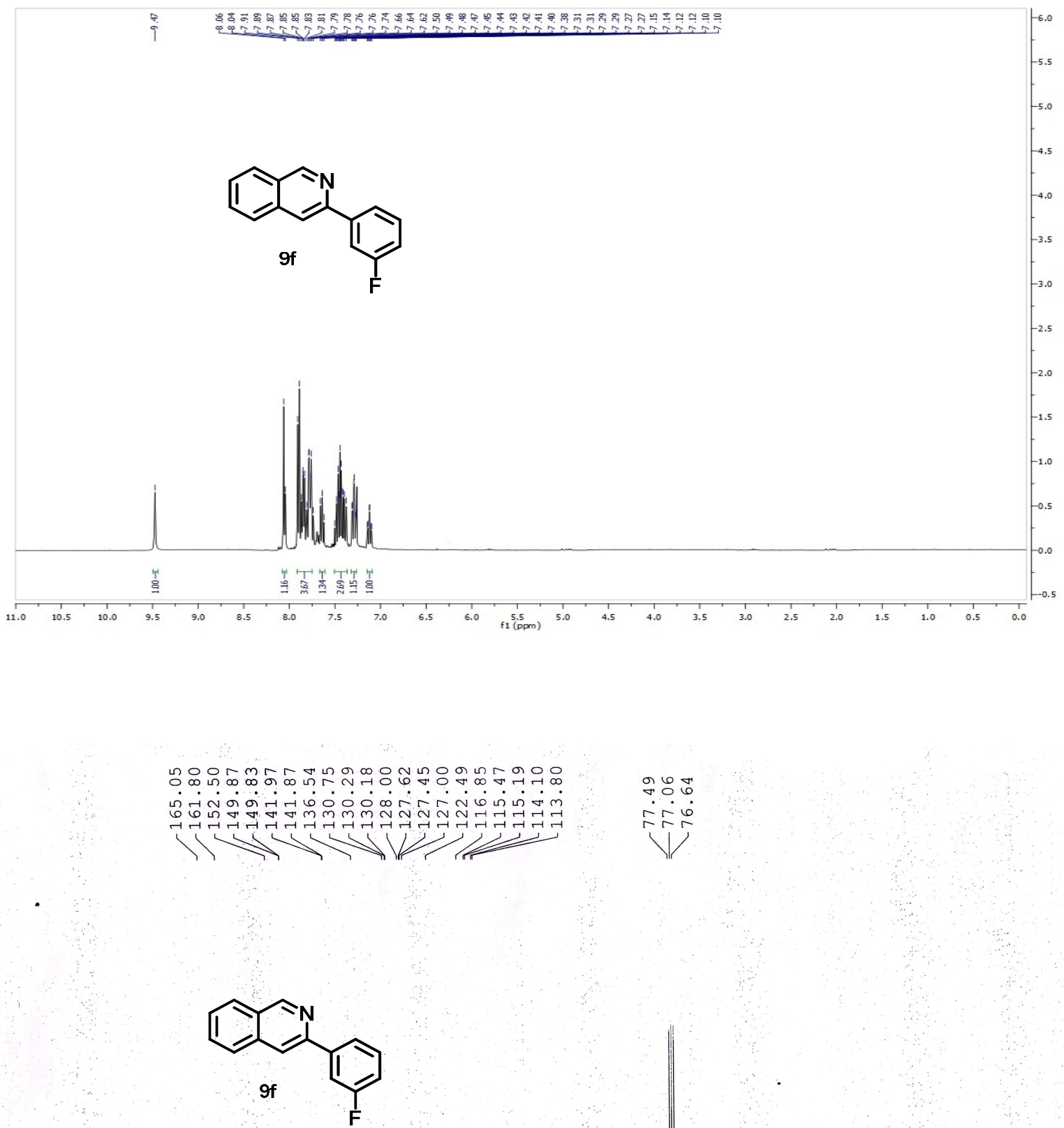

200 
${ }^{1} \mathrm{H}$ NMR (400 MHz) and ${ }^{13} \mathrm{C}$ NMR (100 MHz) spectra of compound 9g in $\mathrm{CDCl}_{3}$.
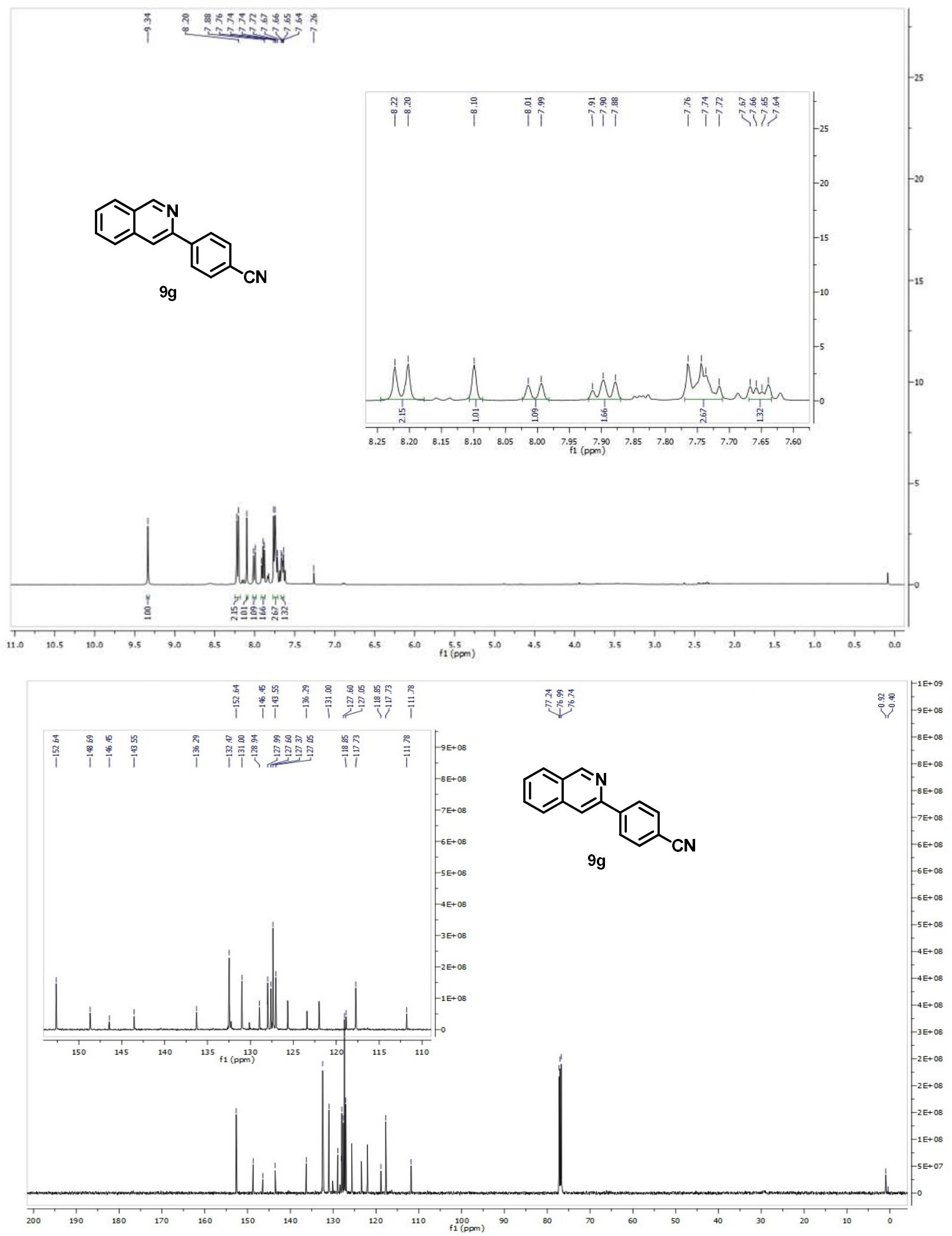
${ }^{1} \mathrm{H}$ NMR (400 MHz) and ${ }^{13} \mathrm{C}$ NMR (75 MHz) spectra of compound 9h in $\mathrm{CDCl}_{3}$.

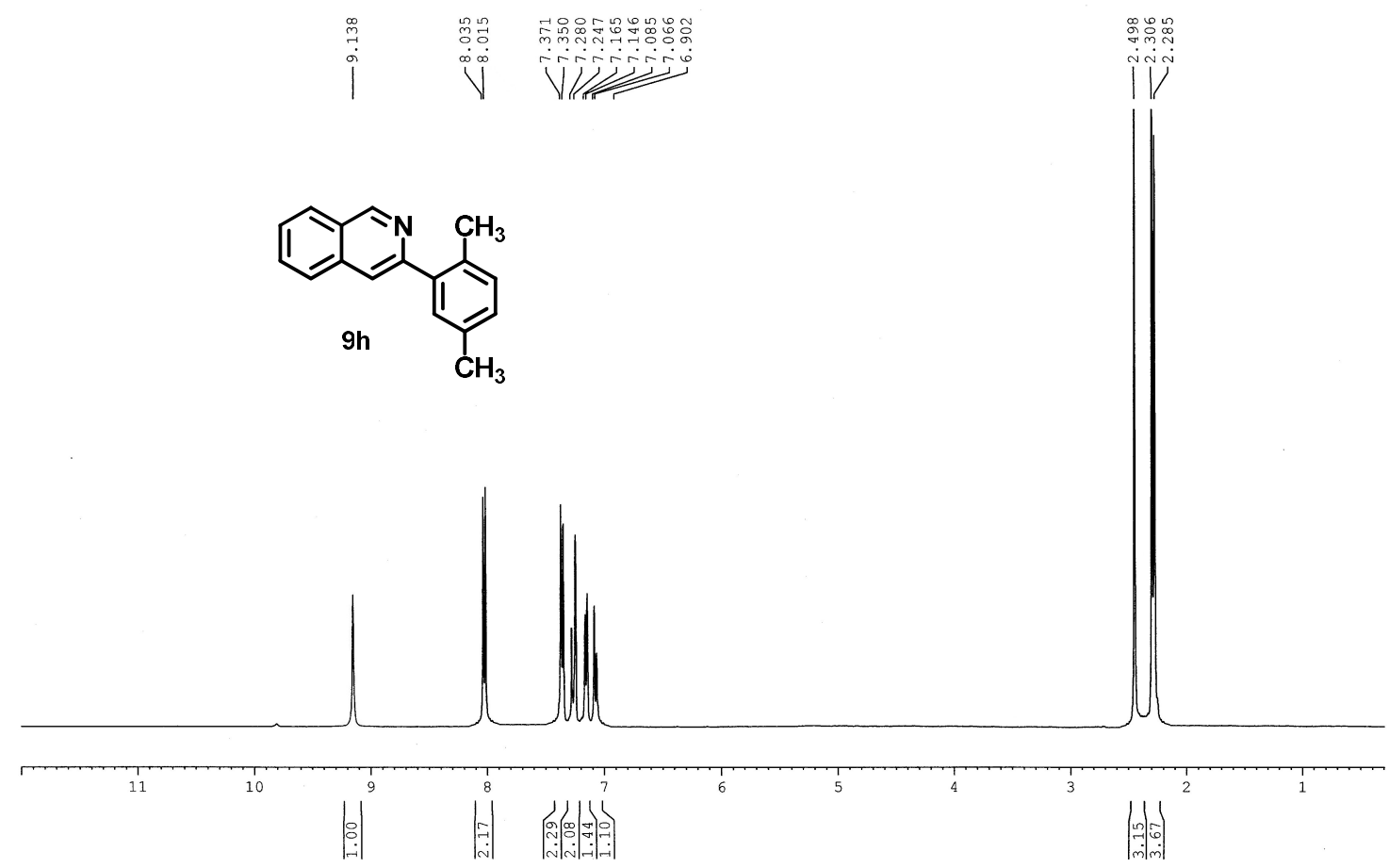

$$
\mathrm{H}_{3}
$$

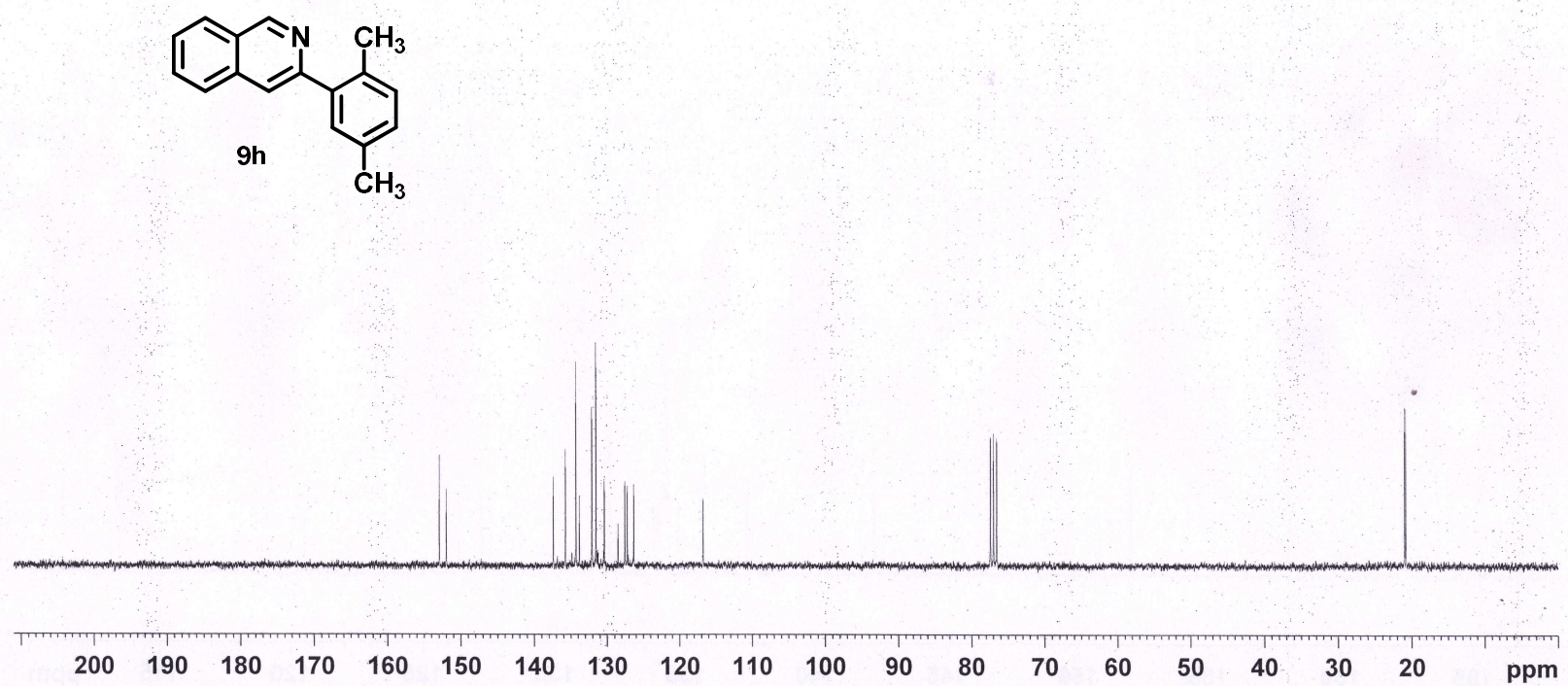

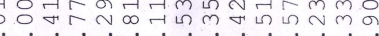

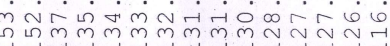

11 W

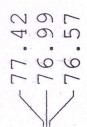

-12
$0 \%$
ํ.
ํ. 
${ }^{1} \mathrm{H}$ NMR (400 MHz) and ${ }^{13} \mathrm{C}$ NMR (100 MHz) spectra of compound 9i in $\mathrm{CDCl}_{3}$.<smiles>COc1ccccc1-c1cc2ccccc2cn1</smiles>
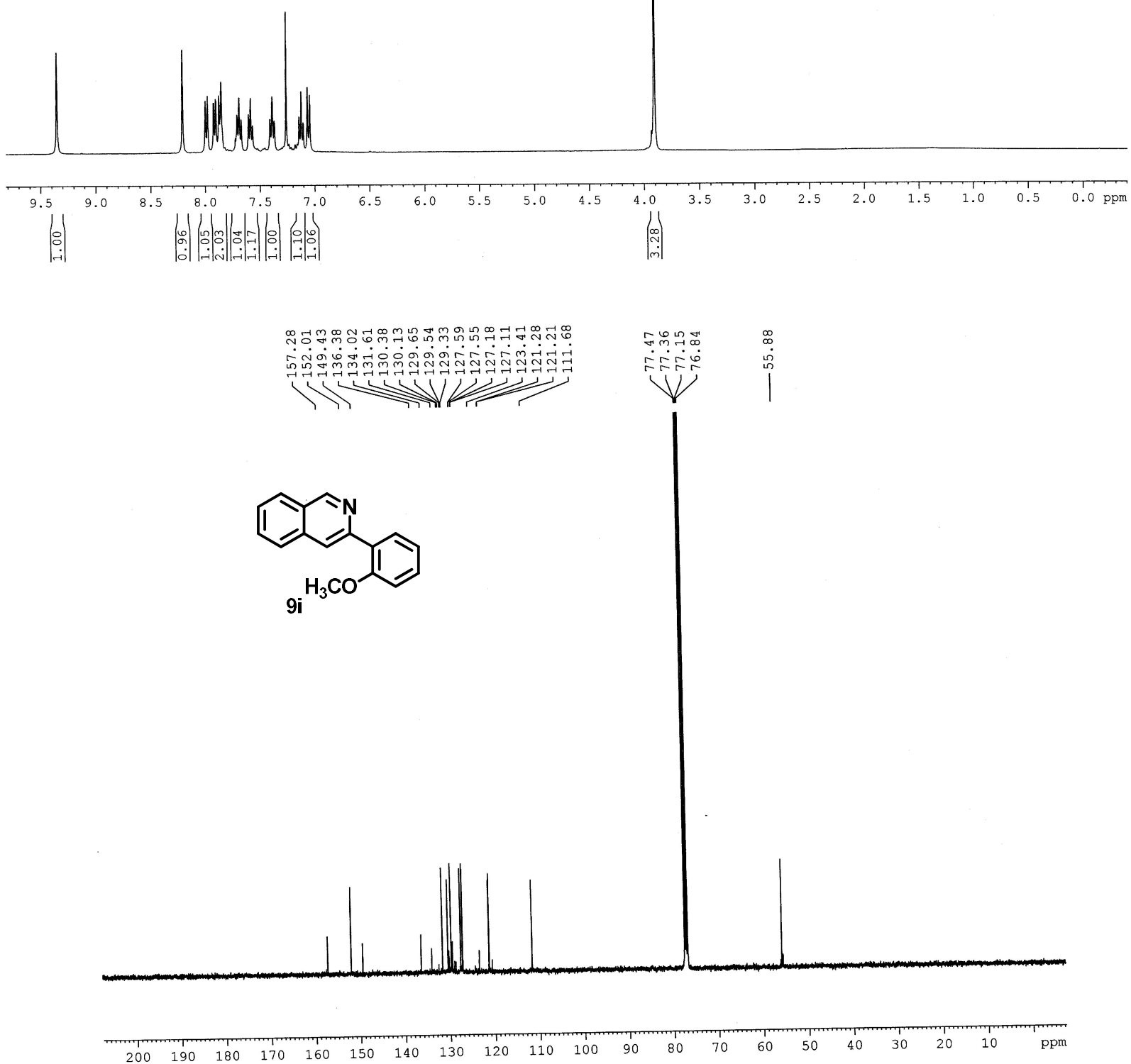

\section{S-51}


${ }^{1} \mathrm{H}$ NMR (400 MHz) and ${ }^{13} \mathrm{C}$ NMR (100 MHz) spectra of compound jj in $\mathrm{CDCl}_{3}$.
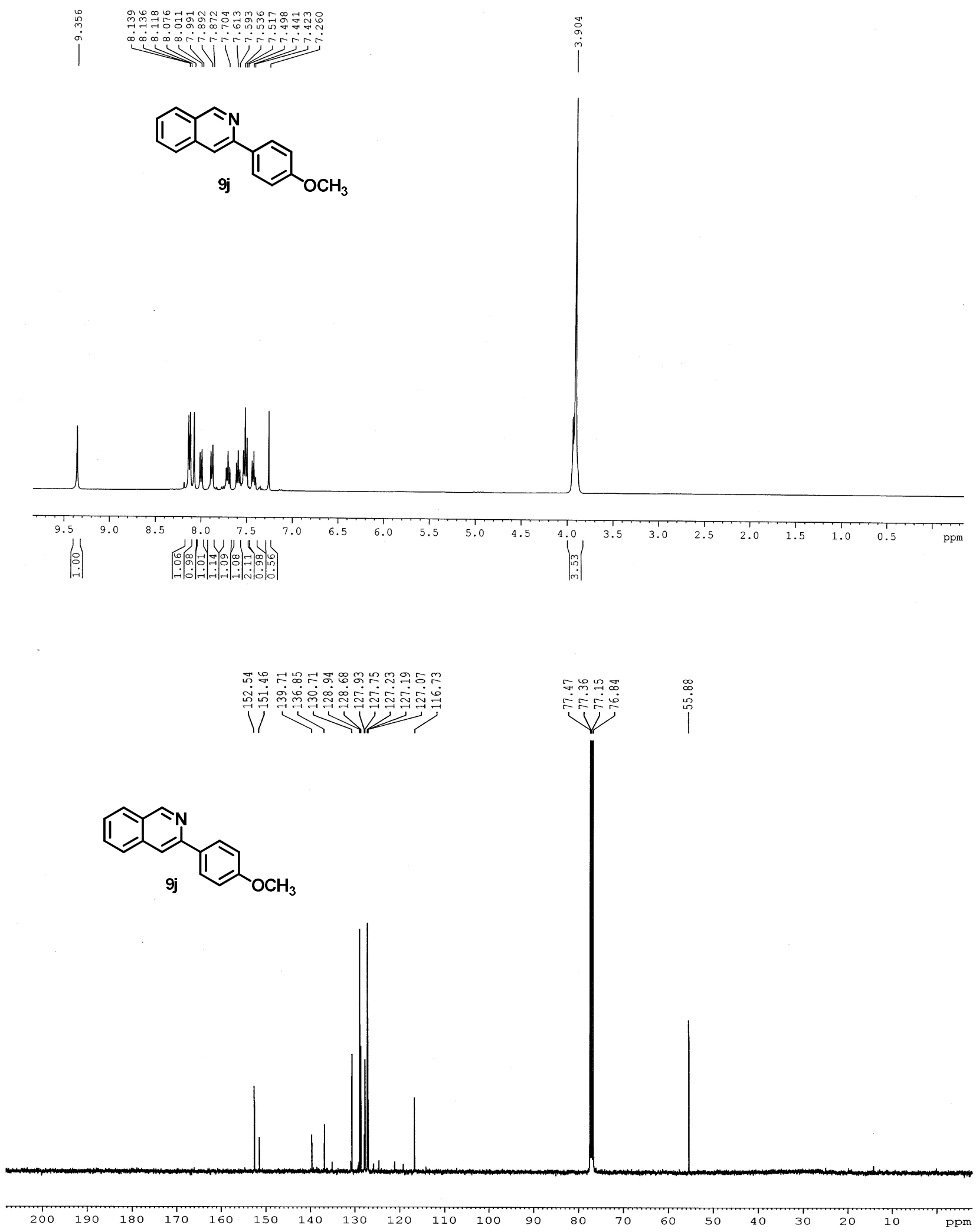

S-52 
${ }^{1} \mathrm{H}$ NMR (400 MHz) and ${ }^{13} \mathrm{C}$ NMR (100 MHz) spectra of compound 9k in $\mathrm{CDCl}_{3}$.
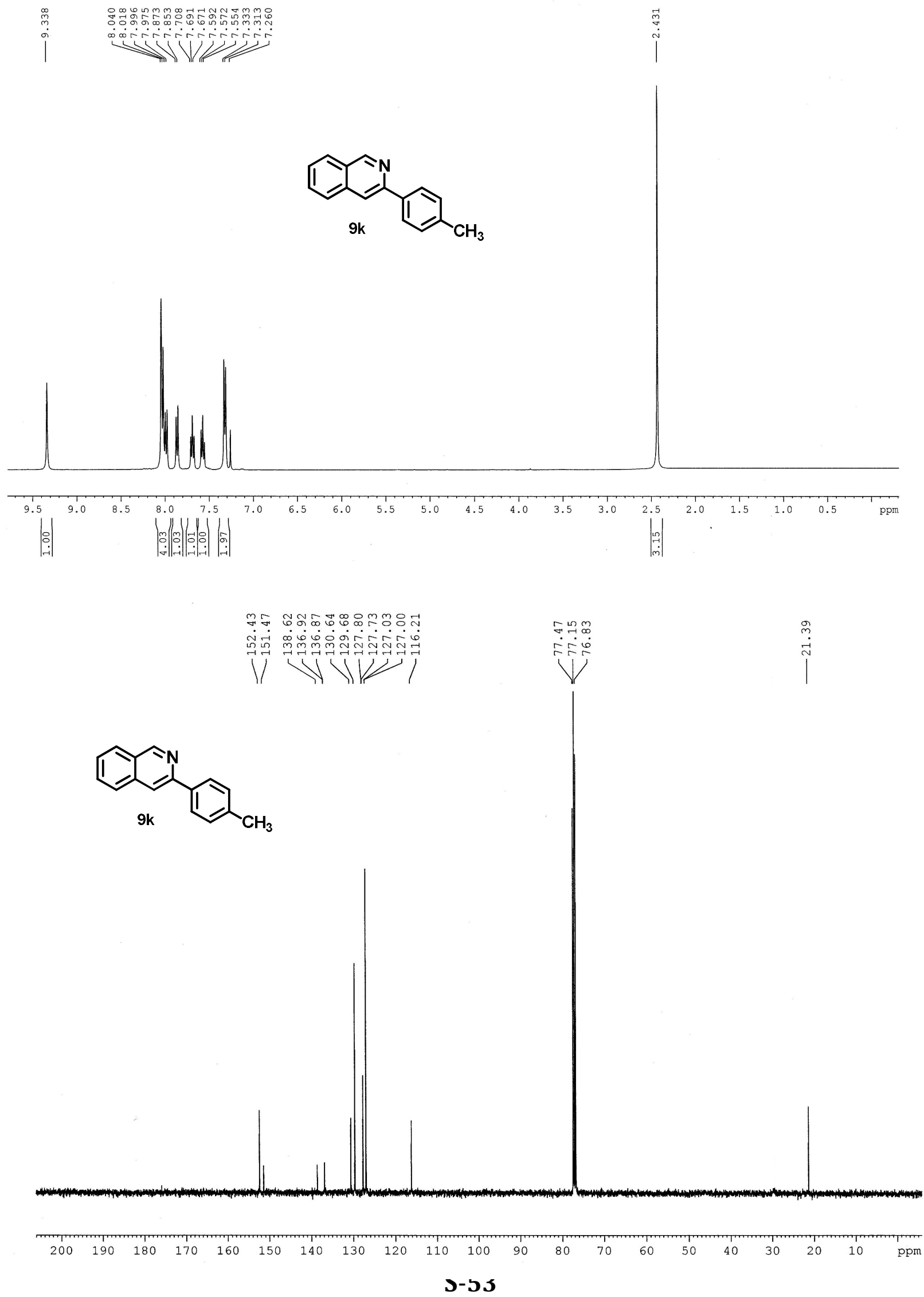
${ }^{1} \mathrm{H}$ NMR (400 MHz) and ${ }^{13} \mathrm{C}$ NMR (100 MHz) spectra of compound 9l in $\mathrm{CDCl}_{3}$.
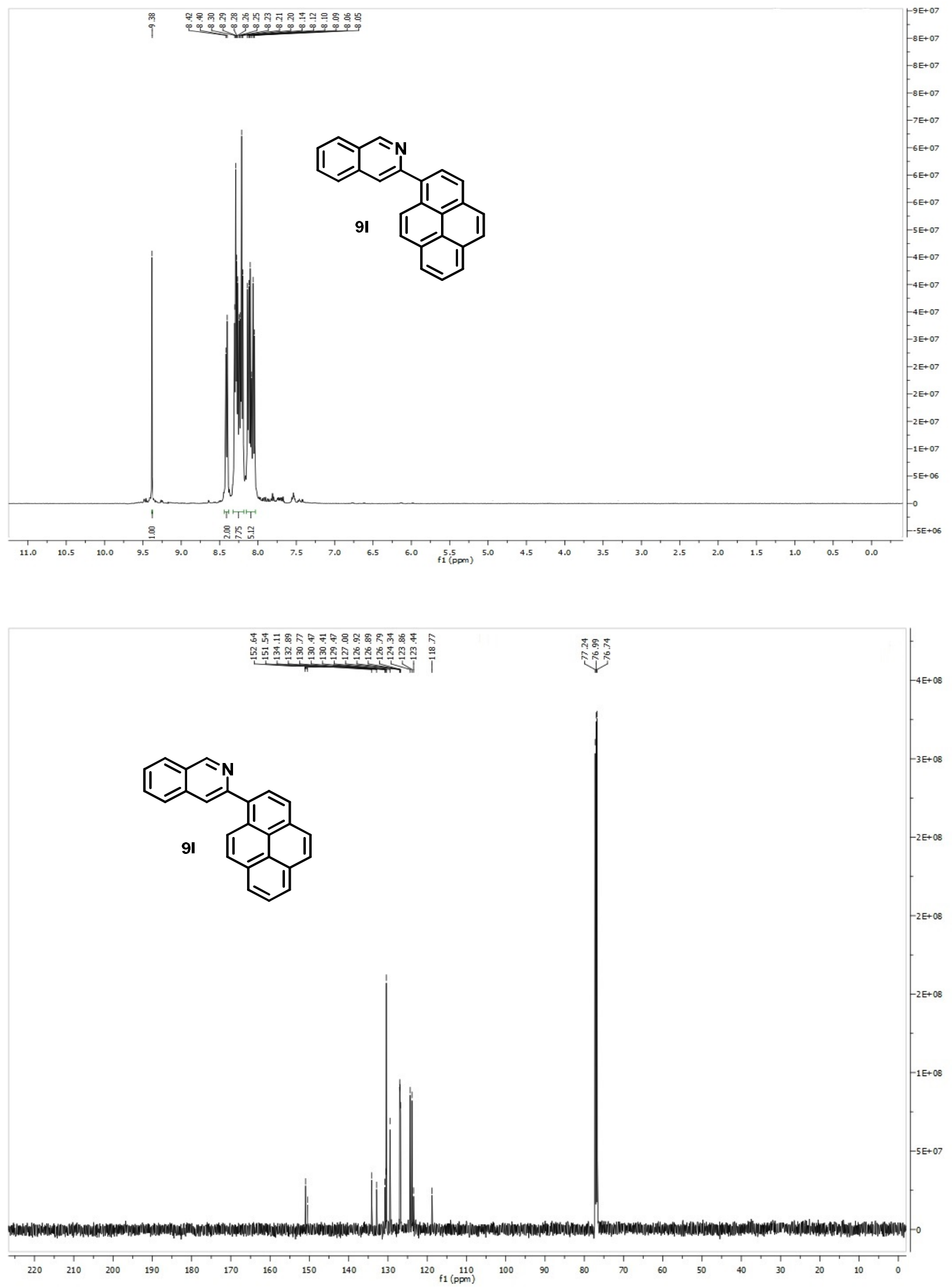

S-54 
${ }^{1} \mathrm{H}$ NMR (300 MHz) and ${ }^{13} \mathrm{C}$ NMR (75 MHz) spectra of compound $9 \mathrm{~m}$ in $\mathrm{CDCl}_{3}$.

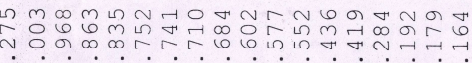

ond

$+1$
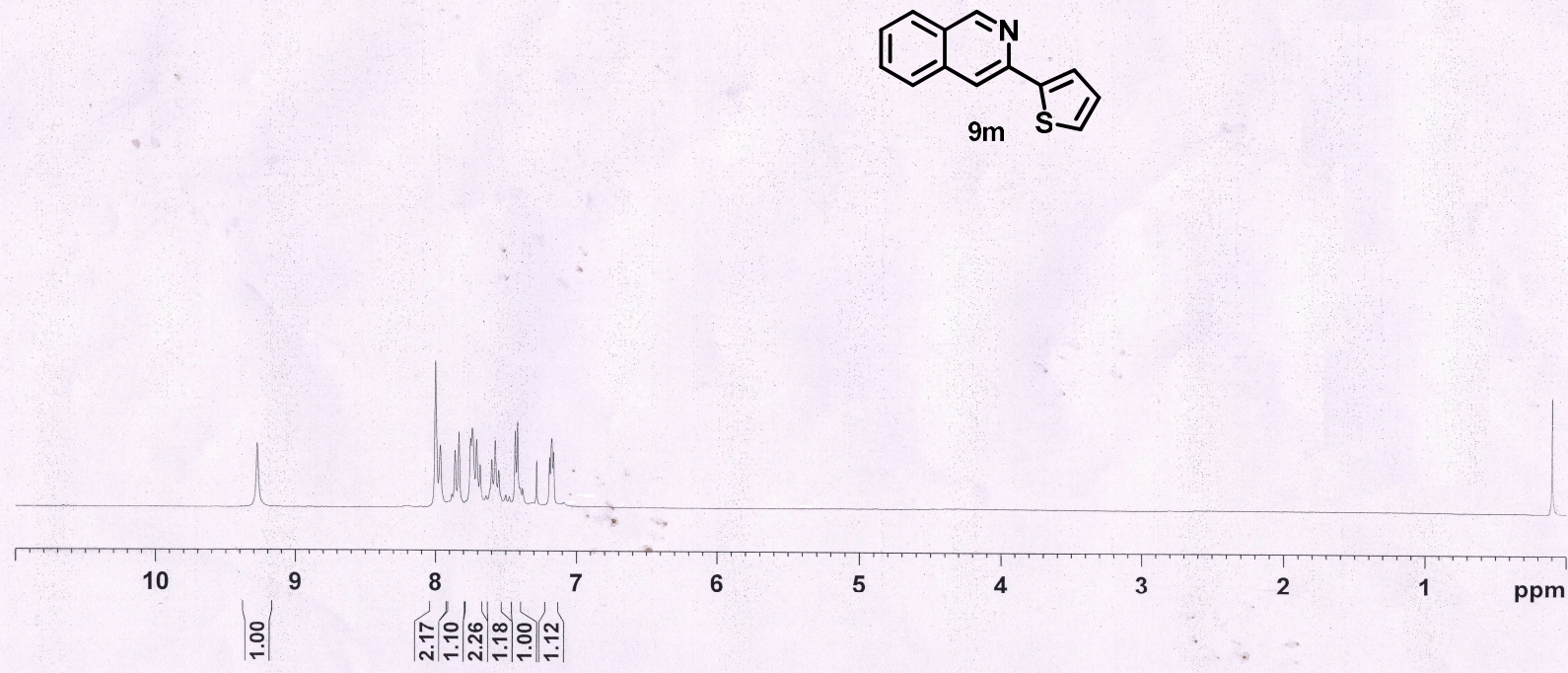

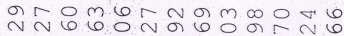

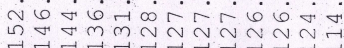

$111<$

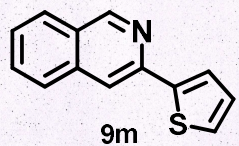

am S

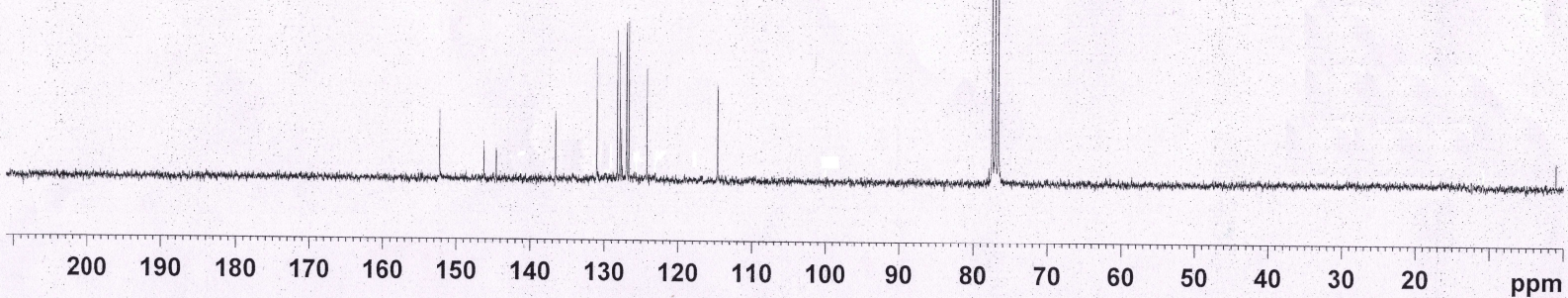

S-55 
${ }^{1} \mathrm{H}$ NMR (400 MHz) and ${ }^{13} \mathrm{C}$ NMR (100 MHz) spectra of compound 9n in $\mathrm{CDCl}_{3}$.
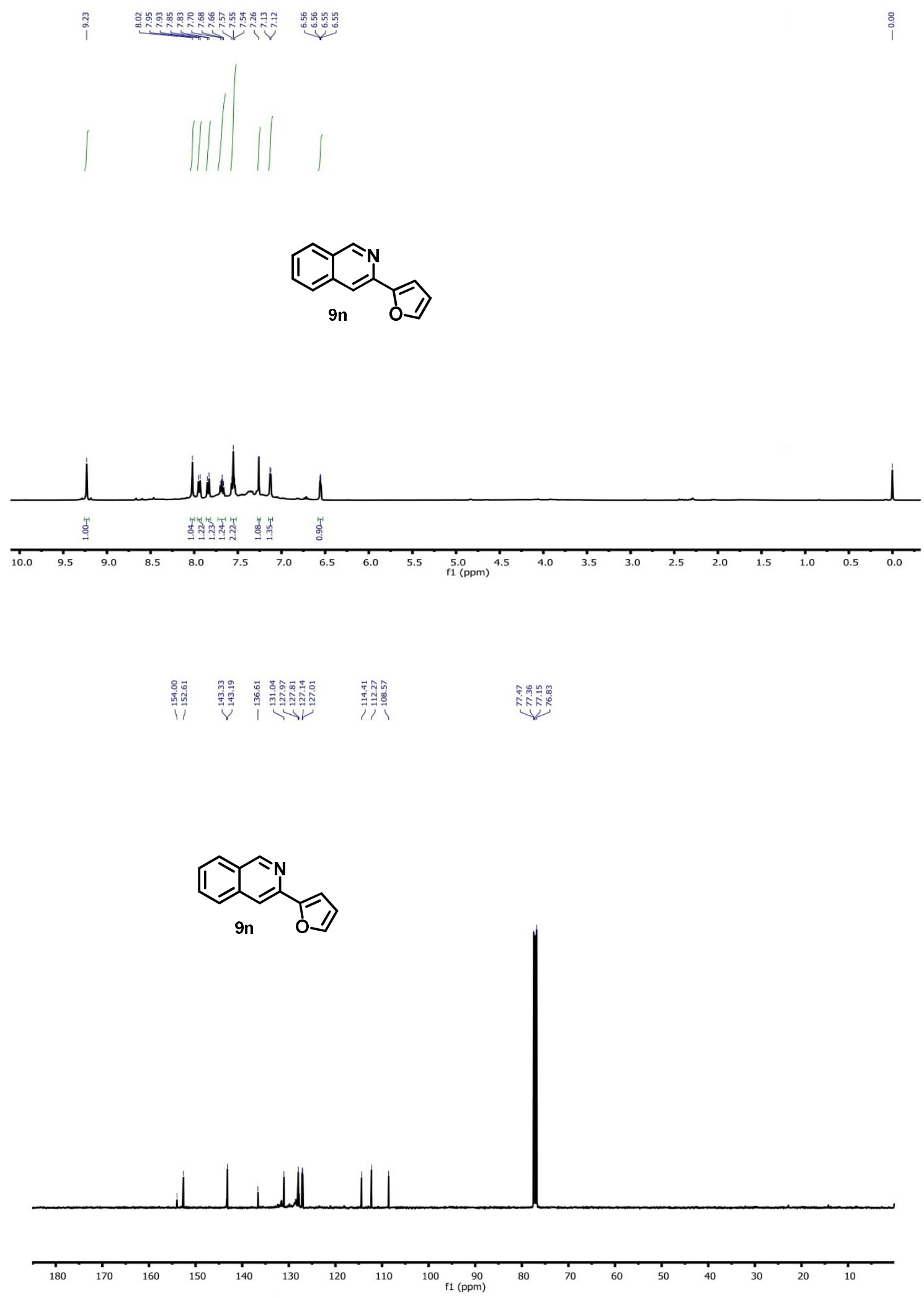
${ }^{1} \mathrm{H}$ NMR (400 MHz) and ${ }^{13} \mathrm{C}$ NMR (100 MHz) spectra of compound 9o in $\mathrm{CDCl}_{3}$.

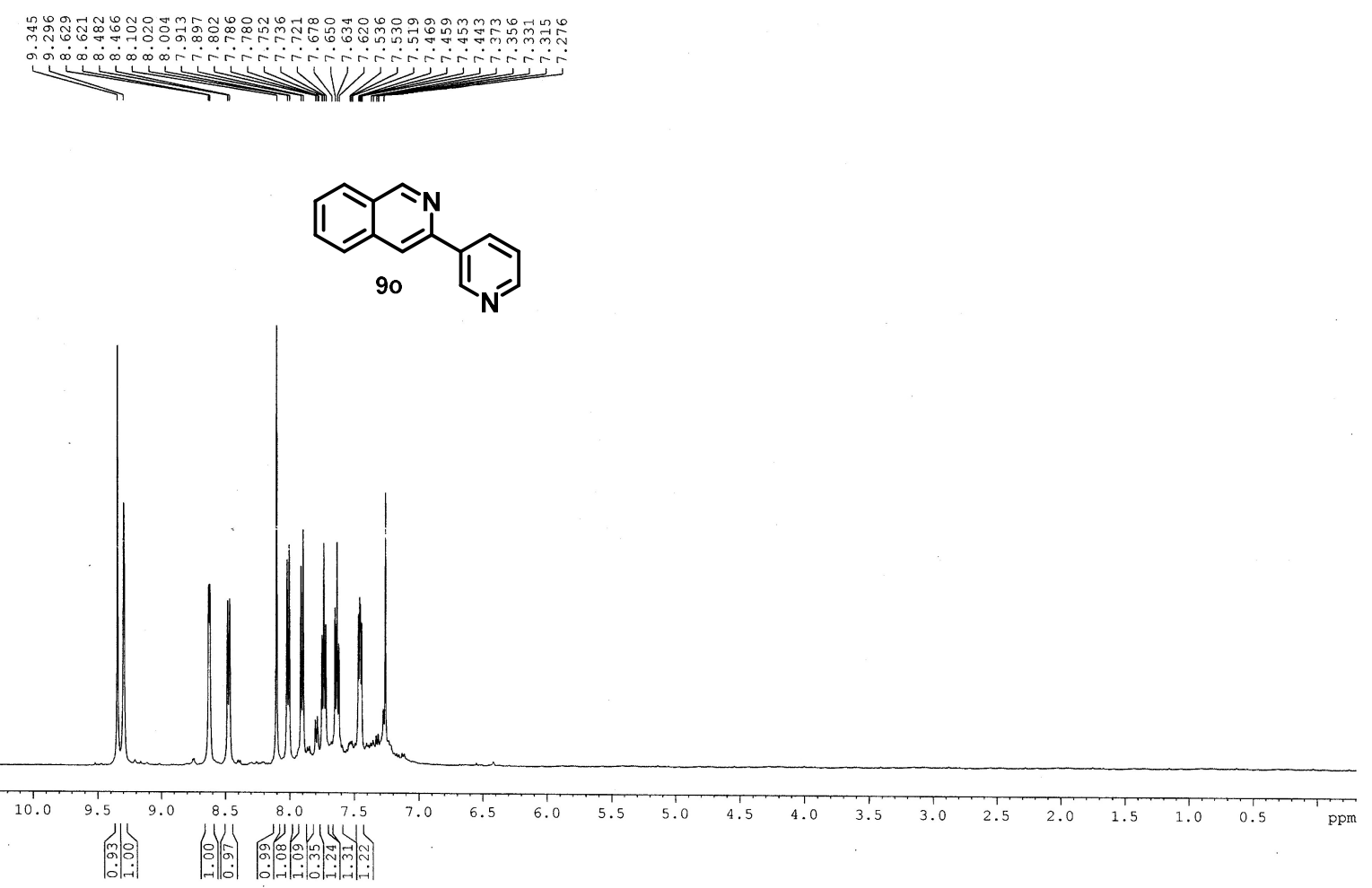

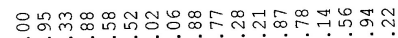

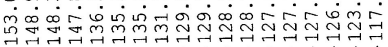

(1)
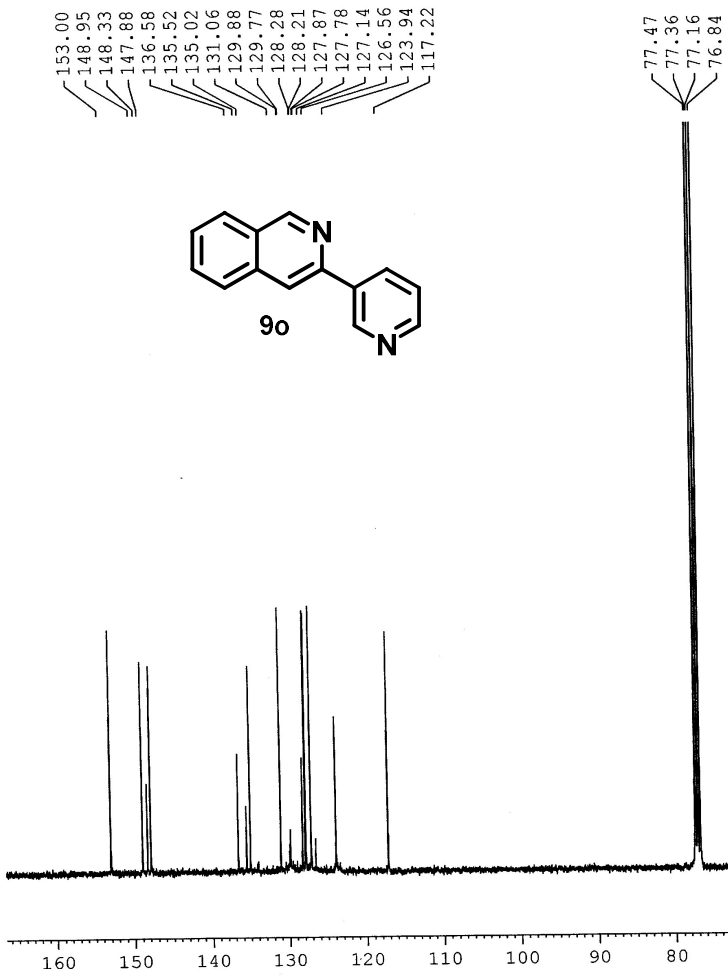

90 
${ }^{1} \mathrm{H}$ NMR (400 MHz) and ${ }^{13} \mathrm{C}$ NMR (100 MHz) spectra of compound 9p in $\mathrm{CDCl}_{3}$.

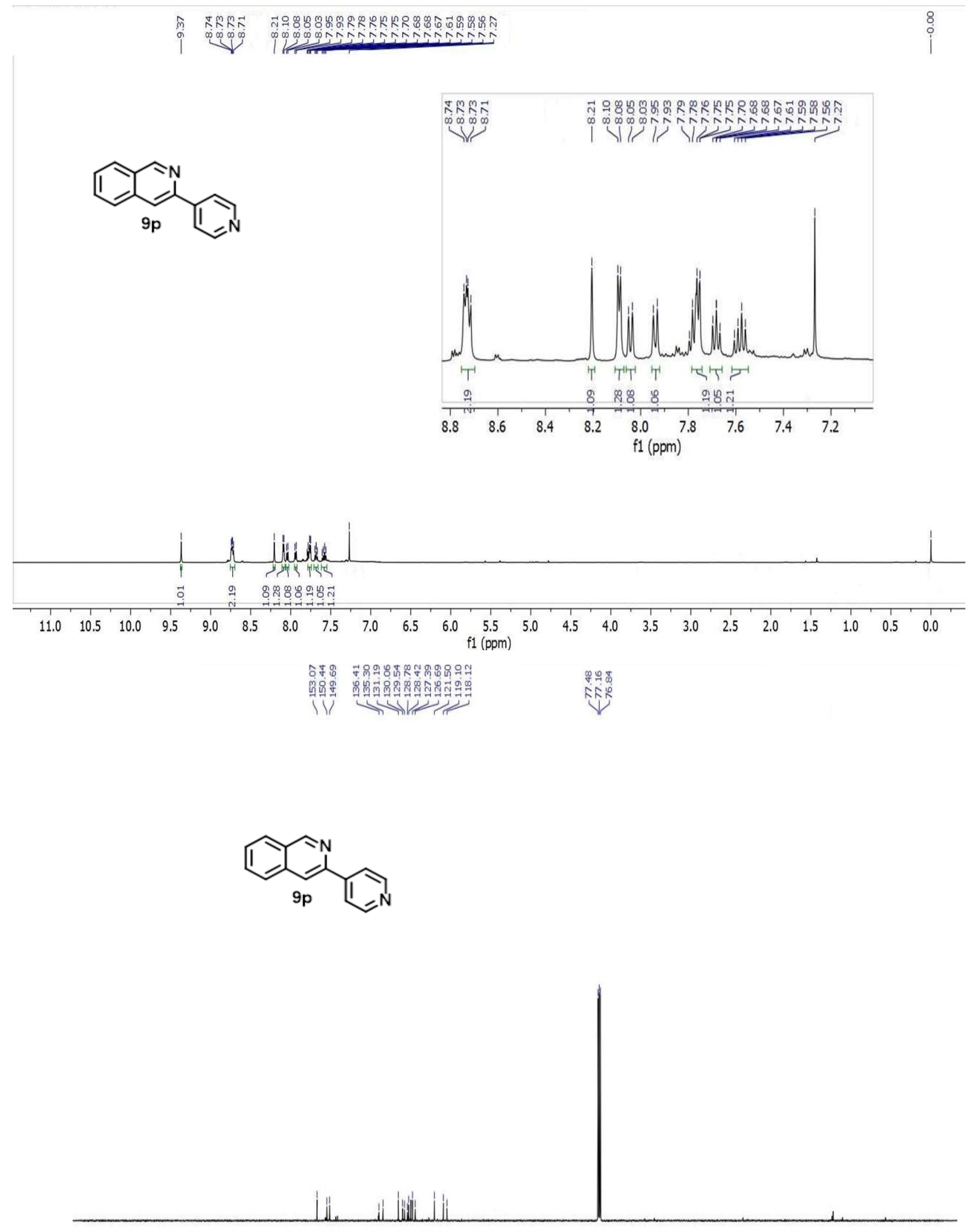

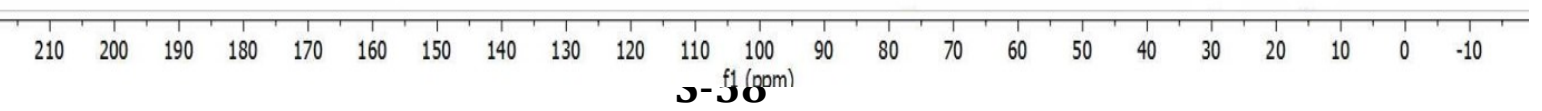


${ }^{1} \mathrm{H}$ NMR (300 MHz) and ${ }^{13} \mathrm{C}$ NMR (75 MHz) spectra of compound 9q in $\mathrm{CDCl}_{3}$.
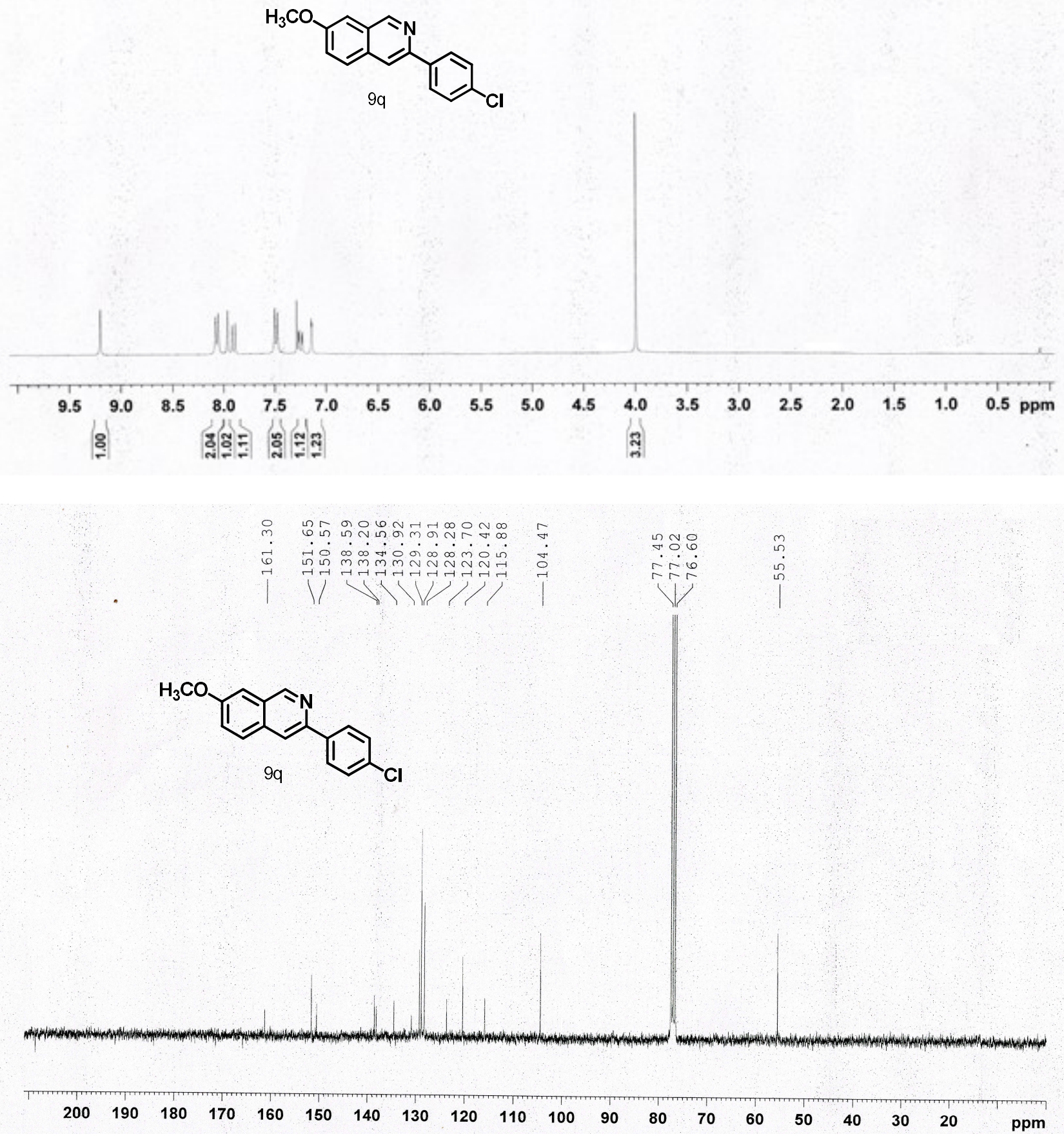
Section VIII: X-ray characterization data summery of compound 6e

Figure S10: ORTEP diagram of compound 6e (CCDC 2062484). Ellipsoids are drawn at 50\% probability level.
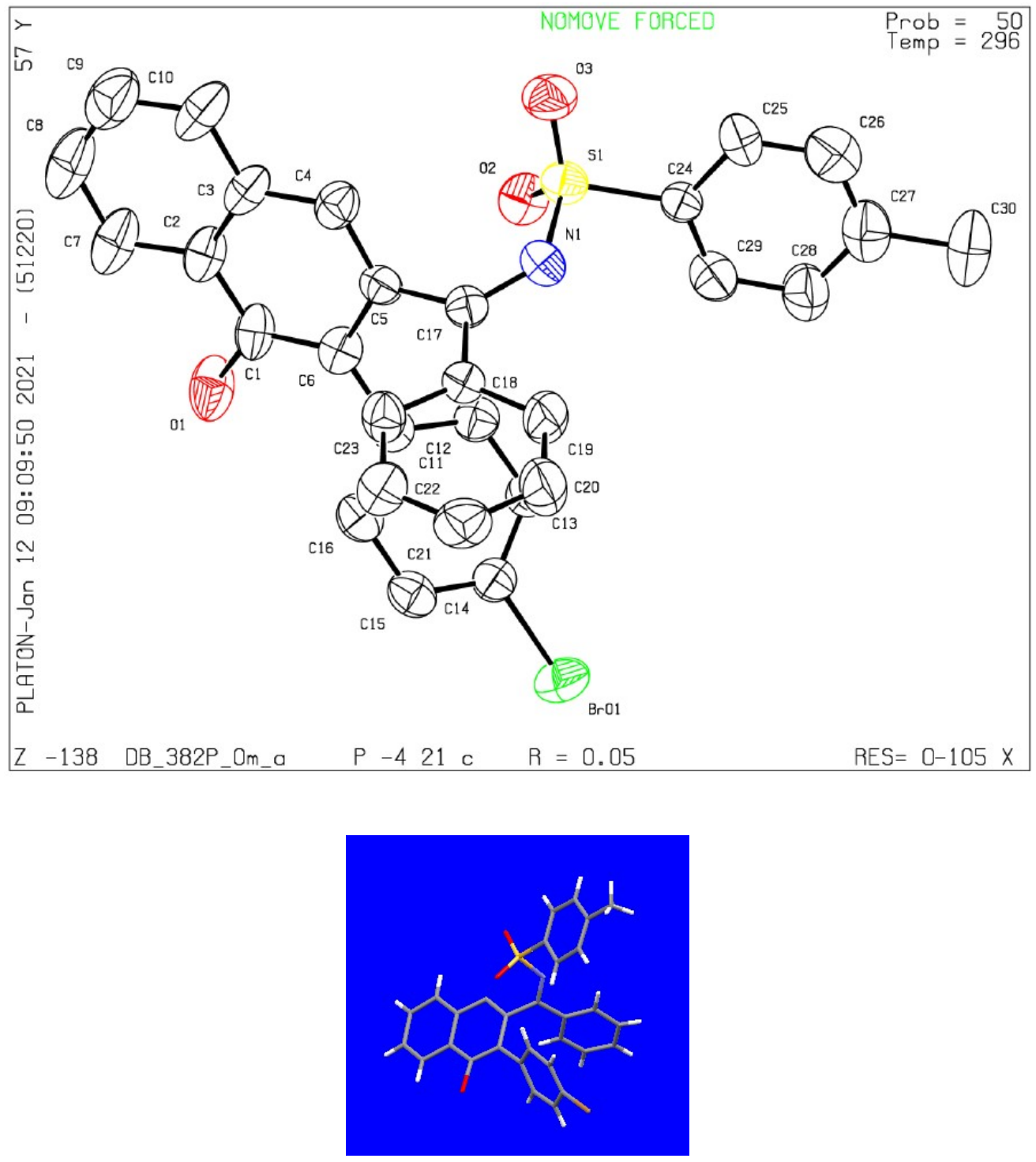
Table S2.Crystal data and structure refinement for $6 \mathrm{e}$

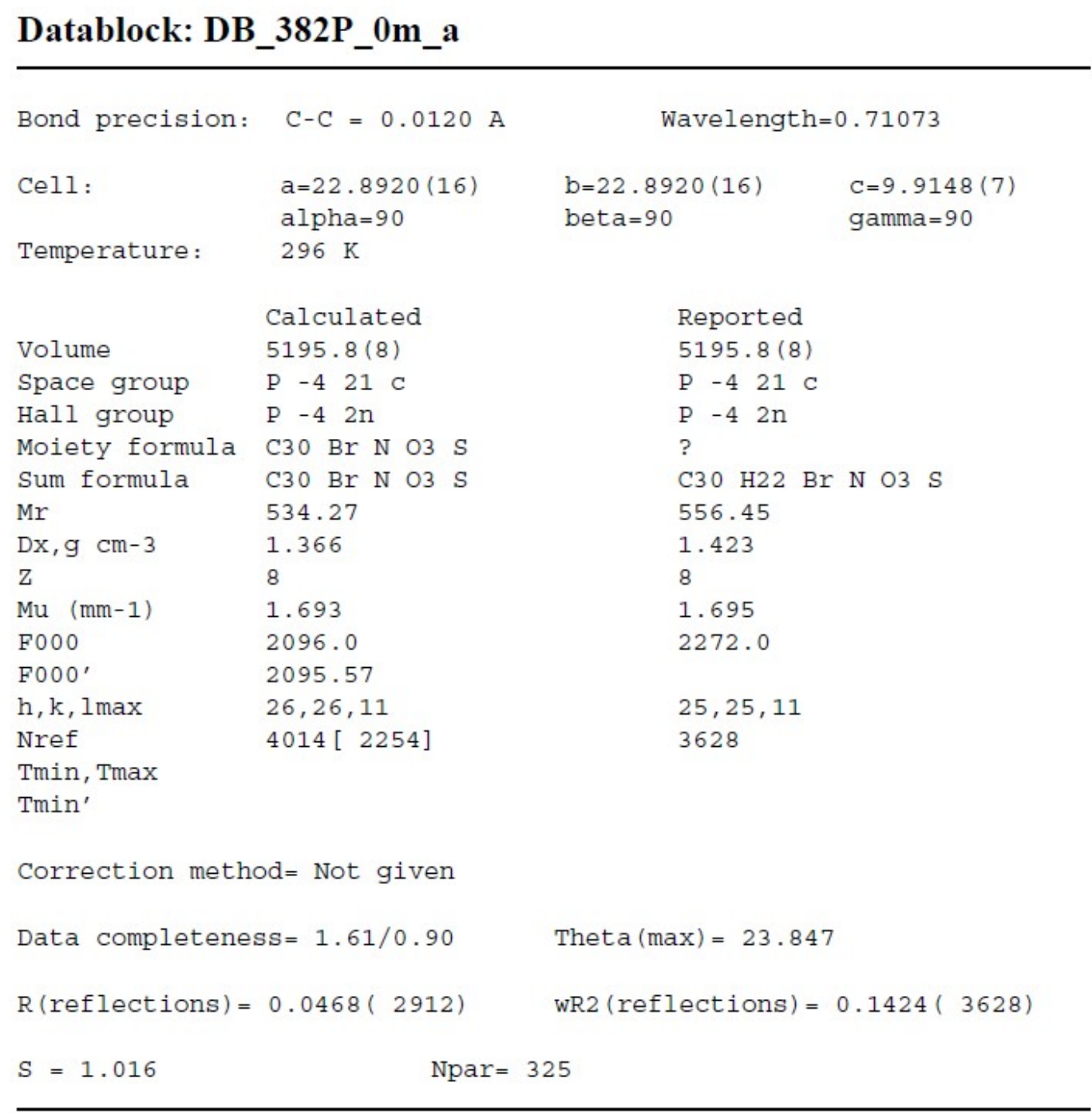


Section IX: X-ray characterization data summery of compound 9b

Figure S11: ORTEP diagram of compound 9b (CCDC 1923004). Ellipsoids are drawn at 50\% probability level.
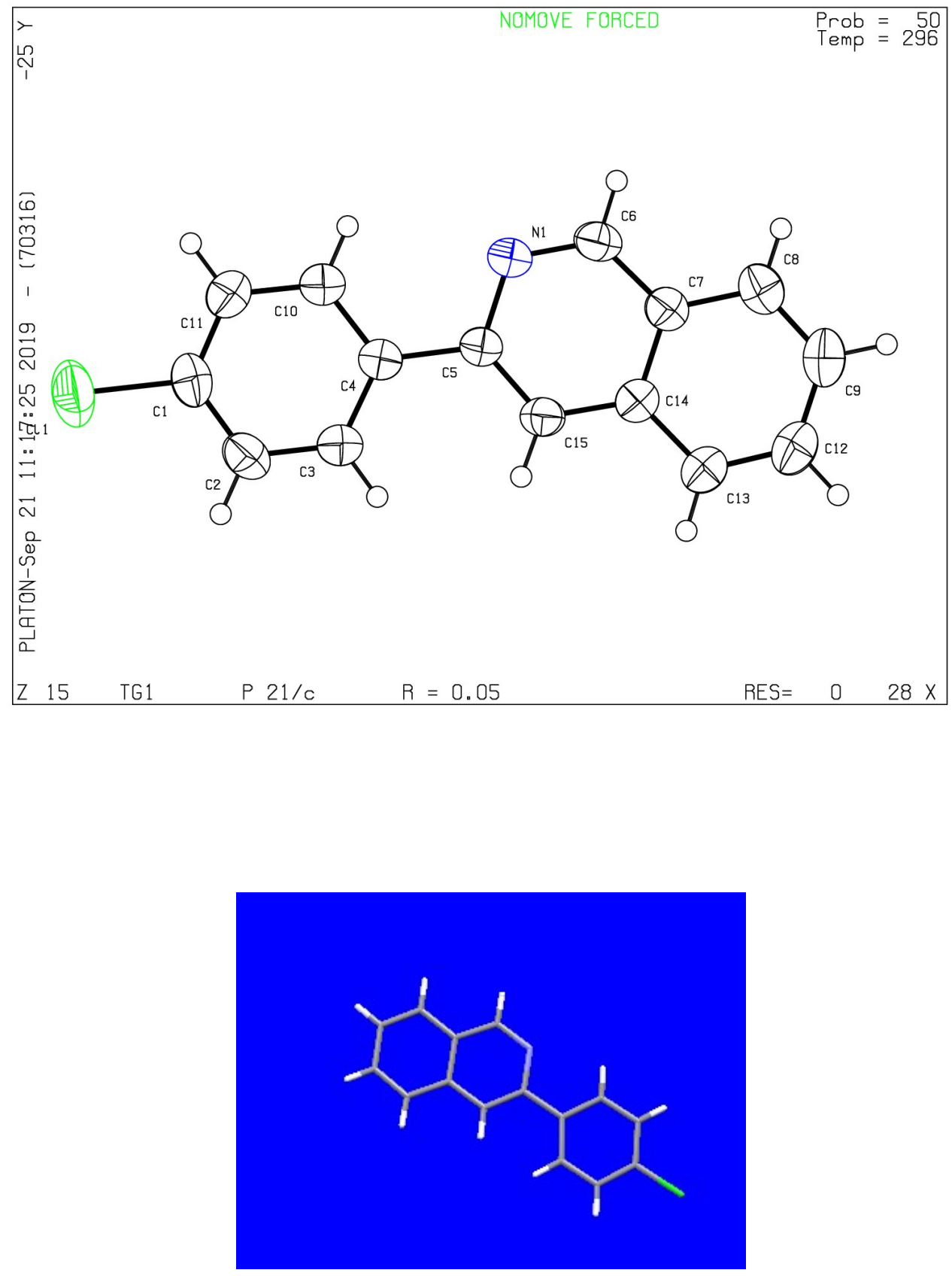
Table S3: Crystal data and structure refinement for $\mathbf{9 b ( C C D C ~ 1 9 2 3 0 0 4 ) : ~}$

\begin{tabular}{|c|c|c|c|}
\hline Bond precision: & $\mathrm{C}-\mathrm{C}=0.0032 \mathrm{~A}$ & Wavelength: & $h=0.71073$ \\
\hline Cell: & $\begin{array}{l}a=13.505(12) \\
a l p h a=90\end{array}$ & $\begin{array}{l}\mathrm{b}=5.878(5) \\
\text { bet } a=106.71(3)\end{array}$ & $\begin{array}{l}C=15.156(13) \\
\text { gamma }=90\end{array}$ \\
\hline Temperature: & $296 \mathrm{~K}$ & & \\
\hline & Calculated & Reported & \\
\hline Volume & $1152.3(17)$ & $1152.4(18$ & \\
\hline Space group & p $21 / c$ & p 21/c & \\
\hline Hall group & $-\mathrm{p} 2 \mathrm{ybc}$ & $-\mathrm{p} 2 \mathrm{ybc}$ & \\
\hline Moiety formula & $\mathrm{C} 15 \mathrm{H} 10 \mathrm{Cl} \mathrm{N}$ & $\mathrm{C} 15 \mathrm{H} 10 \mathrm{C}$ & $\mathrm{ClN}$ \\
\hline Sum formula & $\mathrm{C} 15 \mathrm{H} 10 \mathrm{Cl} \mathrm{N}$ & $\mathrm{C} 15 \mathrm{H} 10 \mathrm{C}$ & $\mathrm{ClN}$ \\
\hline $\mathrm{Mr}$ & 239.69 & 239.69 & \\
\hline $\mathrm{Dx}, \mathrm{g} \mathrm{cm}-3$ & 1.382 & 1.382 & \\
\hline $\mathrm{z}$ & 4 & 4 & \\
\hline $\mathrm{Mu}(\mathrm{mm}-1)$ & 0.304 & 0.304 & \\
\hline F000 & 496.0 & 496.0 & \\
\hline F000' & 496.75 & & \\
\hline$h, k, 1 \max$ & $18,7,20$ & $18,7,19$ & \\
\hline Nref & 2958 & 2832 & \\
\hline Tmin, Tmax & & & \\
\hline Tmin' & & & \\
\hline Correction meth & od $=$ Not given & & \\
\hline Data completenes & $s s=0.957$ & Theta $(\max )=28.68$ & \\
\hline$R$ (reflections) $=$ & $0.0470(1487)$ & wR2 (reflections) $=$ & $=0.1351(2832)$ \\
\hline$S=1.013$ & Npar & 155 & \\
\hline
\end{tabular}

\section{Crystal structure experimental protocol:}

Single crystal of compound $\mathbf{6 e}$ and $\mathbf{9 b}$ were mounted on a Bruker-AXS SMART APEX II diffractometer equipped with a graphite monochromator and $\operatorname{Mo~} \mathrm{K} \alpha(\lambda=0.71073 \AA)$ radiation. The crystal was placed $60 \mathrm{~mm}$ from the CCDC, and frames (360) were measured with a counting time of $6 \mathrm{~s}$. The structures were solved using the Patterson method using the SHELXS 97 program. Non-hydrogen atoms were refined with independent anisotropic displacement parameters, while difference Fourier synthesis and leastsquares refinement showed the positions of any remaining non-hydrogen atoms. The non-hydrogen atoms were refined with anisotropic thermal parameters. Successful convergence was indicated by the maximum shift/error of 0.001 for the last cycle of the least-squares refinement.All other crystallographic details such as $\mathrm{h}, \mathrm{k}, 1$ ranges, $2 \theta$ ranges, and R-factors can be found above (Table S2 and S3).

\section{Reference}

1. (a) M. He and J. W. Bode, Enantioselective, NHC-Catalyzed Bicyclo- $\beta$-Lactam Formation via Direct Annulations of Enals and Unsaturated N-Sulfonyl Ketimines. J. Am. Chem. Soc., 2008, 130, 418-419.

(b) C. Simal, T. Lebl, A. M. Z. Slawin and A. D. Smith, Dihydropyridones: Catalytic Asymmetric Synthesis, N - to C - Sulfonyl Transfer, and Derivatizations. Angew. Chem. Int. Ed., 2012, 51, 3653-3657

2. S. Morales, F. G. Guijarro, J. L. G. Ruano and M. B. Cid, A General Aminocatalytic Method for the Synthesis of Aldimines. J. Am. Chem. Soc., 2014, 136, 1082-1089.

3. X. Wu, G. Ding, L. Yang, W. Lu, W. Li, Z. Zhang and X. Xie. Alkoxide-Catalyzed Hydrosilylation of Cyclic Imides to Isoquinolines via Tandem Reduction and Rearrangement. Org. Lett., 2018, 18, 5610-5613.

4. V. K. Tiwari, N. Kamal and M. Kapur, One Substrate, Two Modes of C-H Functionalization: A Metal-Controlled Site-Selectivity Switch in C-H Arylation Reactions. Org. Lett., 2017, 19, 262-265. 